\title{
Induction voltage adders and the induction accelerator family
}

\author{
Ian D. Smith \\ Titan Pulse Sciences Division, 2700 Merced Street, San Leandro, California 94577, USA
}

(Received 19 October 2003; published 14 June 2004)

\begin{abstract}
Induction voltage adders (IVA) and induction accelerators of various types are described and their principles and advantages are discussed. The designs and technologies used in the various subsections and components of high-current IVAs are described. Some features of the pulse power that drives IVAs are discussed. Two representative high-current IVAs are briefly described, and characteristics of other IVAs with different features are mentioned. Some other IVA and induction accelerator variants that could be considered are also suggested.
\end{abstract}

DOI: 10.1103/PhysRevSTAB.7.064801

PACS numbers: $84.70 .+p$

\section{INTRODUCTION}

The induction voltage adder (IVA) is a method of connecting drive circuits in a series so that their voltages add while restricting their summed voltages to a small central region that is enclosed at a slightly larger radius by a continuous metal cylinder. The first IVAs were injectors for linear induction accelerators (LIA). The LIA injectors add pulsed (tens of ns) voltages of tens or hundred of kilovolts to apply $>1 \mathrm{MV}$ to an anodecathode gap (diode) and launch a 1-10 kA electron beam. This beam is then accelerated by applying similar, tens to hundreds of $\mathrm{kV}$ voltages to gaps in a metal beam pipe. Ferromagnetic cores allow both the injector and the acceleration path to be contained in a grounded outer cylinder.

From the 1980s on, a new type of IVA was developed that could drive diodes at much higher currents, hundreds of $\mathrm{kA}$, still using pulses as short as tens of ns. This required much lower inductance structures connecting the pulse generators to the diode. Low inductance implies high electric fields, and these fields were accommodated by using magnetic insulation or sometimes other techniques. It is these high current IVAs that are the main subject of this article.

The sequence of the sections of this article is as follows: Sec. II first illustrates the difference between the IVA and the LIA. Then it describes in general terms the different members of the "induction accelerator" family. These include designs that use "air cores" instead of ferromagnetic cores, including 100\% efficient designs based on bipolar pulses, and accelerators in which the beam is recirculated. Throughout Sec. II we discuss the different physical principles used in the various accelerators and consider some new possible configurations.

Section III is devoted to the technology used in the high-current IVA. We begin (Sec. III A) by explaining the advantages of IVAs over other methods of connecting drive circuits in series and over full-voltage drivers. Then we describe the technology used in various parts of the voltage adder (Secs. III B and IIIC) and some characteristics of the pulse power needed and used to drive the adder (Sec. III D).

Section IV describes representative actual IVAs.

This article attempts to sum up the work of many people on IVAs, in particular, at Titan Pulse Sciences Division, formerly Pulse Sciences, Inc., and Sandia National Laboratories, Albuquerque; to recognize the previous work on LIAs by many other people at Lawrence Livermore National Laboratory, Lawrence Berkeley National Laboratory, and the National Bureau of Standards; and to recognize other contributions worldwide. The significant characteristic features of IVAs described in this article have been originated by many people too numerous to name individually, and many more people have contributed in the project teams that put the IVAs into practice. The author hopes that this blanket acknowledgement will be acceptable.

\section{THE INDUCTION VOLTAGE ADDER AND THE INDUCTION ACCELERATOR FAMILY}

\section{A. The induction voltage adder: features and functions}

Figure 1 illustrates the essential topology and features of the "adder" portion of an IVA system. The adder itself is formed by a series of $N$ identical, cylindrically symmetric cells ${ }^{1}$ that are pillbox shaped and have one circular face in contact with a face of the next cell. The case $N=3$ is illustrated. The cells are closed toroids, except for a gap around the azimuth of the inside cylindrical surface, and therefore the adder assembled from the cells has a cylindrical on-axis region, referred to as the bore, which has a series of annular gaps regularly spaced on its cylindrical surface.

The outer cylindrical surfaces of the cells form a grounded metal cylinder with closed ends that is the

\footnotetext{
${ }^{1}$ Sometimes called "cavities." In this article, the term "cells" originally coined in induction accelerators is retained for historical reasons and to preserve the term cavity for the part of the cell used for inductive isolation.
} 


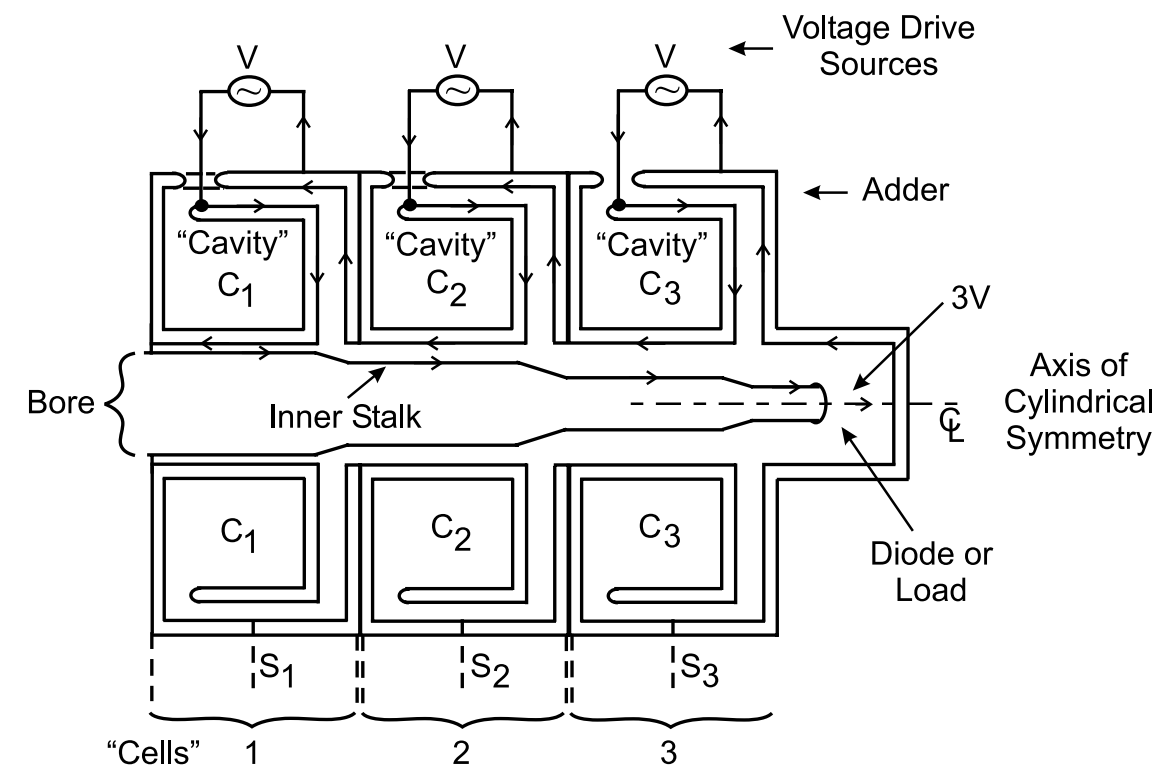

Note: (1) if outer cylindrical adder surface were split at sections S1, S2, and S3, current path and voltage addition would be unaffected; (2) with S1, S2, and S3 connected, summed voltage $3 \mathrm{~V}$ does not appear outside central bore; cavity in each cell then loads the cell voltage drive source.

FIG. 1. The induction voltage adder (three stage). Schematic shows that the current path (indicated by arrows) connects the voltage sources directly in series with the load.

external surface of the adder. The outer cylindrical surface of each cell has at least one port through which passes a high voltage driver connection that delivers a pulse voltage, $V$, across the annular gap in the bore and causes a current, $I$, to flow into the bore. A conducting stalk is mounted from the center of one end of the bore, and this stalk threads the bore; the pulse voltages delivered to each cell thus add along this stalk, to deliver a voltage, $N \times V$, to a gap between the tip of the stalk and the other end of the bore. A current, $I$, of particles can be accelerated across this gap, which is referred to as the diode and is the load for the adder.

The beam of particles formed in the diode can be extracted though an aperture or window and transported away from the adder; alternatively the particles can be immediately deposited in a target at the end face of the adder, when electrons accelerated across the diode gap strike a high atomic number anode and create $\mathrm{x}$ rays that irradiate a nearby object. Having the diode gap at one end of the adder gives easier access to the particle beam or $x$ rays. In special cases there may be reasons to use two shorter stalks, one extending from each end of the adder, with the diode between the tips of the two stalks, near the center of the adder.

The current path through the high voltage drivers and the diode load is indicated in Fig. 1 by the arrows. The load current comes directly from the high voltage drivers external to the adder, which are connected directly in series by the cylindrical conductor of the adder bore. Hard connected in this way, the pulse generators drive the load to a voltage $N V$ independent of whether the closed, grounded outer structure of the cells and adder is present or not. The function of the outer structure is to prevent the addition of voltages being seen outside the central bore. The benefits of eliminating high external voltages in this way may include suppression of electromagnetic interference to nearby systems, avoiding electrical breakdown, eliminating energy wasted in charging external stray capacitances, and the ability to make parallel connections upstream in the driver circuits. The penalty is that closing each cell connects a toroidal conducting cavity $\left(C_{1}, C_{2}\right.$, and $C_{3}$ in Fig. 1) across the high voltage driver that penetrates it and thus tends to load that driver. The parasitic current that the cavity draws from the driver is usually too large if the cavity contains only material of permeability one, and so the parasitic current is therefore usually reduced by filling the cavity as far as possible with ferromagnetic material surrounding the bore; the ferromagnetic material is in the form of annular "cores." For these cores to remain effective throughout the drive pulse, they must not saturate. Therefore the radial cross sectional area $(A)$ of cores, as viewed in Fig. 1, multiplied by the flux density swing $(\Delta B)$ that the core material can support without saturation, must be greater than the voltsecond product $(V T)$ of the pulse (see Sec. IIIC).

As we will see in later sections, the cores limit the parasitic currents in IVAs to typically a few kA to $10 \mathrm{kA}$ average during the pulse. IVAs typically have 
diode currents of $50 \mathrm{kA}$ or much more, and losses in the cores are therefore quite tolerable in terms of efficiency.

Another benefit of the IVA is that electrical access to both electrodes of the diode is easy because from the external world both appear to be at ground. Note that if this is not a consideration then no closed cavity or core material is needed in the first cell, as long as the stalk that threads the bore can be mounted directly from the conductors bringing the input from the high voltage drivers of the first cell. But it may in any case be mechanically convenient to support the stalk from ground, so the first cell usually has a closed cavity and cores also.

The term IVA is sometimes said to stand for inductive voltage adder. The author prefers induction, because this term has always been used for the linear induction accelerator, which uses very similar cells (see Sec. II B). Also, cavities filled with ferromagnetic material do not behave like inductors, but more like energy-absorbing resistors.

There are many design variations possible in an IVA. Some of the more fundamental choices are the following. (1) The high voltage pulses, usually tens of ns in duration, are not generated in vacuum but in fluid or solid dielectrics and are almost always liquid dielectric. The transitions from the higher density to the vacuum that is needed to accelerate particles in the diode may be made just outside each cell, or inside each cell, or as a single transition at the load end of the adder bore. (2) The cores may be of ferrite or of wound metal tape, usually an amorphous metal. (3) If the central bore is evacuated (the usual case) it may either operate at electric fields below electron emission thresholds or use the magnetic field of the current in the stalk to control the electrons emitted (i.e., "use magnetic insulation"). The demonstration that magnetic insulation worked in an adder, allowing high electric fields to be used, launched the development of the high current IVAs that are the main subject of this article. (4) The polarity of the stalk may be positive or negative in order to drive different types of diode or to accelerate ions or electrons out of the end of the adder. These design options are discussed in Sec. III, and different designs using them are described in Sec. IV.
In addition, there has been recent interest in placing the high voltage drive circuits inside the cell; this is mentioned briefly in Sec. IV. In the remainder of Sec. II we discuss induction systems other than IVAs that have many of the same design features and options. We discuss the relations between them and IVAs, and how their circuits operate.

\section{B. The linear induction accelerator}

The IVA was preceded historically by the LIA [1], Fig. 2. This has the same topology as the IVA except that over most of the length of the series of cells the bore is threaded not by a conductor but by a drifting particle beam of current $I$ (usually electrons in practice). The beam is guided through the adder bore by a magnetic field and is accelerated in turn by each of the gaps in the wall of the bore. At the downstream end of the bore the particles attain a total energy $\mathrm{NeV}$ corresponding to acceleration by a voltage $N V$. The current path is essentially the same as in the IVA; the beam is always surrounded by an equal and opposite current in the wall of the bore, often referred to as an "image current," and this return current crosses the gaps in the bore by flowing through the pulse generators outside the adder.

The first section of an LIA has usually been an IVA, albeit of a simpler, lower power kind than those that will be discussed in Secs. III and IV. The bores of the first few cells of the LIA are threaded by an axial conductor that drives a diode that creates the beam that is injected into the rest of the accelerator. One such topology is illustrated on the left in Fig. 2. The IVA portion of the LIA shown is known as the "injector"; it has sometimes used cells and pulse generators different from those in the main drifting-beam section of the LIA, and in some cases it does not use a LIA at all but a fullvoltage drive circuit [2].

The design of the LIA has the same motivation that was noted for the IVA: it allows the use of many small accelerating voltages instead of one very large one, and it

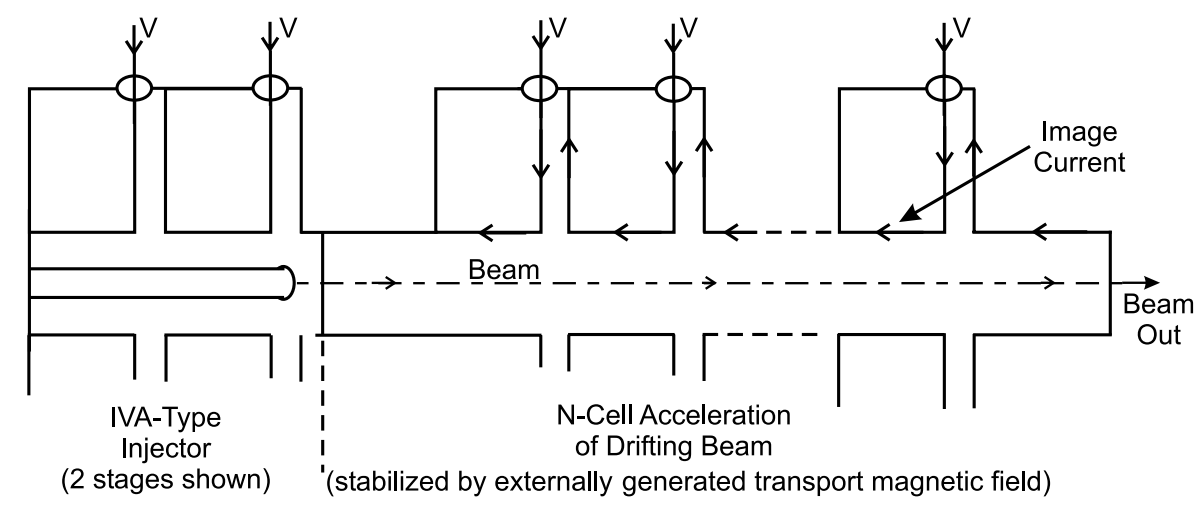

FIG. 2. Schematic of linear induction accelerator. 
confines the total acceleration voltage, suppressing breakdown, losses, and electromagnetic interference.

Currents accelerated in LIAs are limited by instabilities in transport of the drifting beam and usually have not exceeded $10 \mathrm{kA}$. Often they are only a few kA. This means that low-loss core materials are needed in order to make core currents low compared to the beam load; the choices of the core materials will be discussed in Sec. III.

\section{C. "Induction" and voltage transformation}

The term "induction" that is common to the IVA and the LIA refers to the fact that the applied voltage pulse generates induction in the cavity of the cell. This fact is associated with the existence of the closed external conducting cylinder of the adder and hence with the fact that the total voltage appears only in the bore, and these characteristics can be taken to define an induction system.

It has been written that in an LIA the beam is accelerated by $d B / d t$ in the cavities or cores. However, $E$ fields, not $B$ fields, accelerate particles. If there is a closed conducting path in parallel with an acceleration path, then the accelerating $E$ field will also create a $d B / d t$; but the $d B / d t$ is not the cause of the acceleration. In the IVA and LIA the view that $d B / d t$ causes the acceleration seems particularly inappropriate, because in Figs. 1 and 2 the load would be driven or the particles accelerated (in fact perhaps more efficiently) if the closed external structure were not present and no $d B / d t$ existed. The $d B / d t$ is a side effect of limiting the accelerating fields to a volume inside a closed conductor.

The LIA has also been described as a series of 1:1 transformers coupling pulse generators to the beam, and this might equally be said of the IVA. The 1:1 ratio negates the existence of transformation in the sense of transforming voltage or current; it therefore presumably alludes to coupling circuits through the use of cores. But it

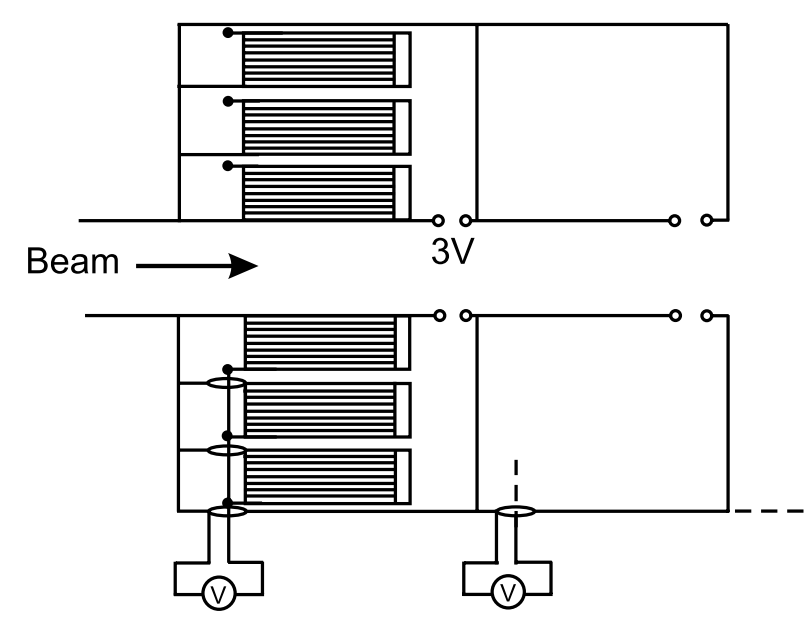

FIG. 3. Schematic of radial segmentation. is clear, as noted in the previous paragraph, that in the LIA and the IVA illustrated the coupling is by hard connection, and the cores are for reduction of parasitic currents, not to couple circuit elements that would not be strongly coupled without the cores. Therefore the term transformer applied to Figs. 1 and 2 seems inappropriate to the author.

However, voltage transformation can in fact be a feature of an IVA (or LIA). All of the three external pulse generators in Fig. 1 could be connected in parallel, to form in effect a single pulse generator driver of voltage $V$ and current $N I=3 I$, without affecting the IVA performance; then $N: 1$ transformation or voltage step-up would have been affected. In this case the external closed conducting structure of the cells could not be completely broken. Inspection shows that two closed cavities $(N-1$ in the general case) must remain closed by the conductors that connect the high voltage feeds in parallel to the single driver. But the path of the load current through the driver remains made up of direct connections.

Cygnus (Ref. [3] and Sec. IV B) is an example of an IVA that uses voltage step-up transformation of this kind. Like Fig. 1, it has three cells but all have a common driver.

Figure 3 illustrates the geometry of a LIA [4] that incorporates transformation in a different way. In each cell the cores are in radial segments driven by a single voltage source, forming a radial voltage adder that can be combined with similar cells to form an axial adder or accelerator. In the case of the two-cell device described in Ref. [4], there were five radial core segments in one cell and four in the other.

\section{Air-core versions}

In the IVA and LIA versions illustrated in Figs. 1 and 2, the induction cavities are filled with magnetic material to reduce the parasitic loss currents that they draw from the pulse generator. But the magnetic material would not be necessary in order to have high electrical efficiency if the adder or accelerator parameters were such that the current produced in the cavity was much less than the beam current because of the inductance of the cavity, even if the cavity was "air cored" (i.e., had permeability one throughout).

To quantify roughly the load or beam currents and pulse durations for which omission of magnetic material might be practical, suppose that the outer radius of the system, $r_{o}$, is about 4 times the bore radius $r_{i}$, and that the cavity occupies a fraction $F=85 \%$ of the cell length. The cavity inductance per meter of system length is then $0.85 \times 200 \ell n 4 \sim 250 \mathrm{nH}$. If the adder voltage or accelerating voltage is equivalent to $E \mathrm{MV}$ per meter of system length (accelerating gradient $=E$ in $\mathrm{MV} / \mathrm{m}$ ), the current in the cavity will rise linearly throughout the pulse duration $t(\mathrm{~ns})$ to a final value $\approx E t / 250(M A)=4 E t$ (kA). The time average of the cavity current during the 
pulse is therefore $2 E t$. The energy lost in the cavity as a fraction of the beam energy is therefore $2 E t / I$, where $I$ $(\mathrm{kA})$ is the beam current. If this quantity is much less than 1, the efficiency may be acceptable; the condition for this is $E t / I \ll 0.5$. The general result is that the wasted energy fraction is $\left[2.5 / F \ell n\left(r_{o} / r_{i}\right)\right] E t / I$.

Examples of this general type of LIA are the isolated Blumlein [5] and Radlac [6]. Both differ from the configuration of Fig. 2, but both are designed to accelerate a beam from one end of a metal enclosure to the other, and neither incorporates magnetic material.

A module of Radlac and part of its neighboring module are illustrated schematically in Fig. 4. Water dielectric triplate pulse forming lines (pfls) extending in from outer radius $r_{3}$, which are radial but occupy only a fraction of the circumference, form two pulses of amplitude $V$ with $E$ fields in opposite directions. One pulse is inverted so that it can be added to the other and the beam is accelerated by the combination of the two, $2 \mathrm{~V}$, at radius $r_{1}$. There are no closed circular cavities, but the inverter drives a local cavity $C_{i}$ and a cavity $C_{e}$ extending between $r_{1}$ and $r_{3}$, both with voltages $V$.

The energy wasted in the cavities $C_{i}$ and $C_{e}$ must be at least as calculated in the paragraph above, with $r_{0}$ taken as the average tank radius $r_{3}$ and $r_{i}$ as the radius $r_{1}$ where the two voltage pulses add. The inefficiency may be greater if the cavity inductances are energized nonuniformly, e.g., because waves do not spread throughout them before the end of the pulse or if electrostatic energy is also present after the pulse.
Note that in Radlac the addition of voltages is not confined to the central bore as in other LIA and IVA designs, but to some degree it is seen at a larger radius $r_{2}$ (Fig. 4). This fact contributes to losses. Still, Radlac is confined in a metal tank at still larger radius $r_{3}$, preventing its fields from extending to larger distance.

Radlac was originally built as an LIA accelerating a beam of about $100 \mathrm{kA}$. Because of difficulties with the magnet coils needed to provide the high fields the transport of such currents requires, Radlac was later tested as a $12 \mathrm{MV}, 100 \mathrm{kA}$ magnetically insulated IVA [7]. The operation was more successful than that of the LIA version, but the loss electrons always associated with selfmagnetic insulation (see Sec. III B) tended to damage the vacuum insulators, which were at small radius. In the original LIA design mode, electrons were confined away from the insulators by the magnetic guide field.

Figure 5 illustrates a possible alternative approach to connecting pulse lines in a series in a large tank without the use of cores. Coaxial water-dielectric pulse lines contained in an oil-filled tank are simply connected in series near the vacuum insulator stack. At this radius they create a wave that fills the region between the bore or beam pipe and the tank with magnetic flux.

Features of interest are (1) the approach can use established pulse line technology such as the fast-charged pfls used in many of the IVAs described in Sec. III, (2) by using oil dielectric outside the pulse lines themselves it minimizes wasted electrostatic energy, and (3) because of the high wave velocity in the oil it should tend to fill the

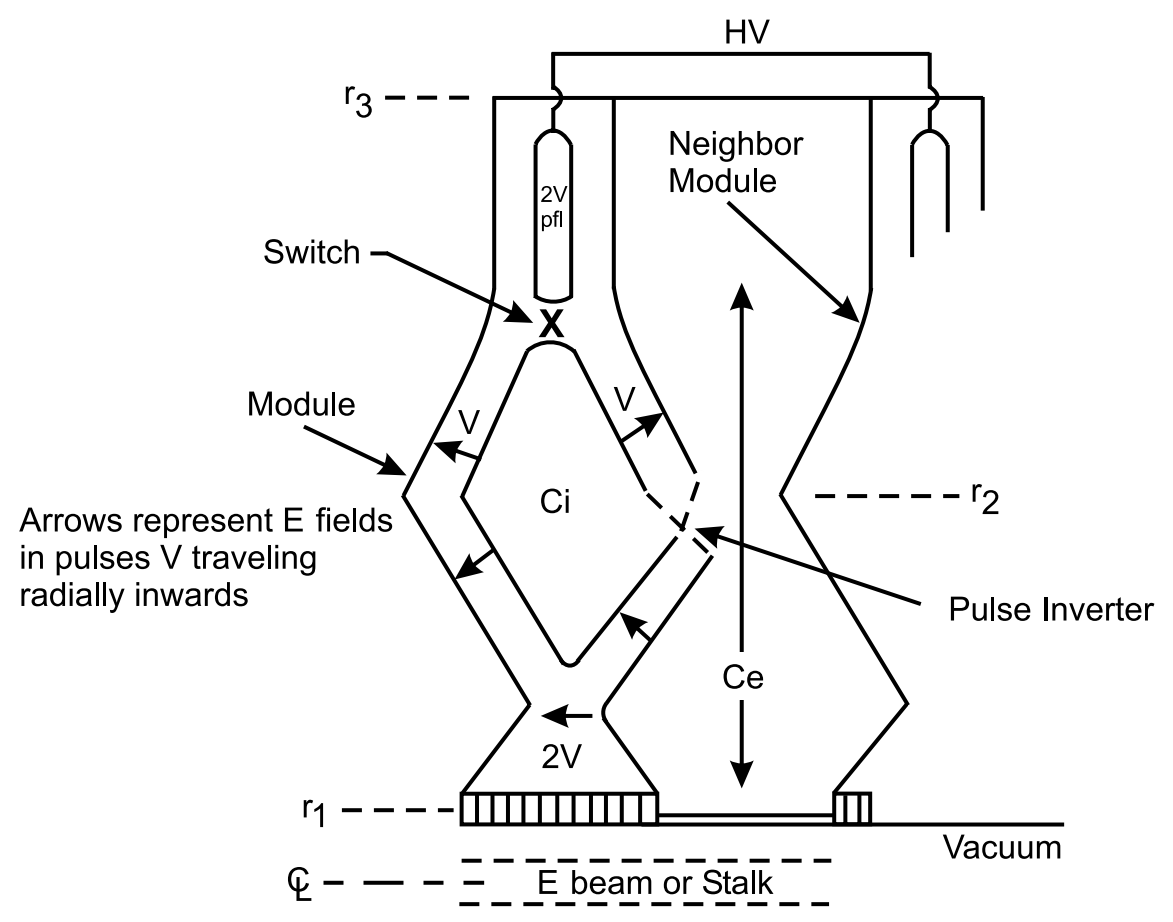

FIG. 4. Module of the air-core LIA Radlac or IVA Radlac smile. 


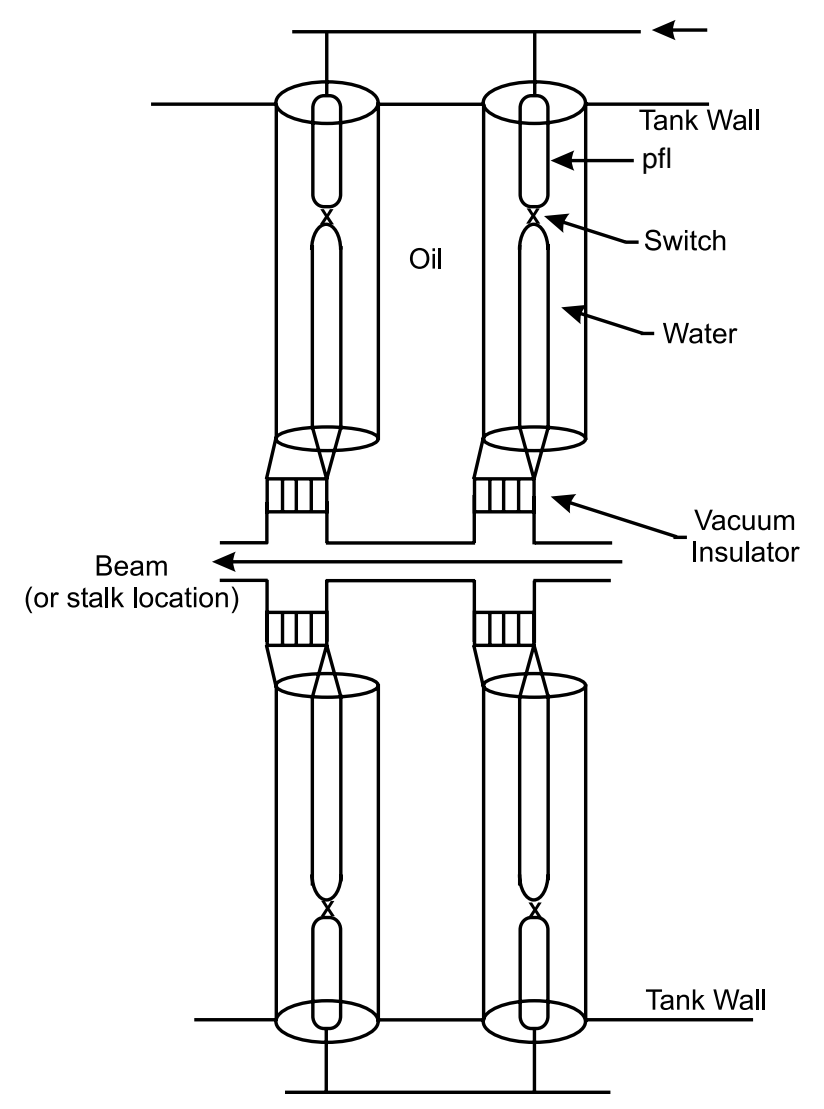

FIG. 5. Schematic of unipolar LIA (or IVA) using coax pfls in an inductive tank.

inductance between the central voltage-addition region and the grounded outer tank with a fairly uniform flux density, again minimizing wasted energy. Suppose as an example that the pulse duration is $30 \mathrm{~ns}$, that the tank is filled with oil, and that a reflection from the tank wall is seen at the end of the pulse. This requires the tank to be $20 \mathrm{ft}$ in diameter and for an 18-in. bore typical for an IVA the inductance is about $0.5 \mu \mathrm{H} / \mathrm{m}$. For a $1.5 \mathrm{MV} / \mathrm{m}$ "gradient," the current associated with the total voltage wave will be about $1.5 / 0.5 \times 30=90 \mathrm{kA}$, probably less on average throughout the pulse. For a $200 \mathrm{kA}$ IVA this might represent an acceptable efficiency; cost is saved since no cores are needed. Providing the total 250$300 \mathrm{kA}$ current requires two $\sim 50 \mathrm{~cm}$ diameter pulse lines in parallel, as shown in Fig. 5.

Clearly this example is not likely to be useful for a LIA, because most LIAs operate at much lower currents.

The isolated Blumlein (Fig. 6) is a module for a type of LIA known as a "radial pulse line" accelerator. It consists of a Blumlein with circular symmetry driving a central accelerating gap, with the output conductors forming a shorted cavity or cell (in this case the cavity forms the whole cell) that does not perturb the output pulse, because the cavity and the charged conductor are extended outwards far enough that the effect of the short is not seen at the accelerating gap until the end of the pulse. Because

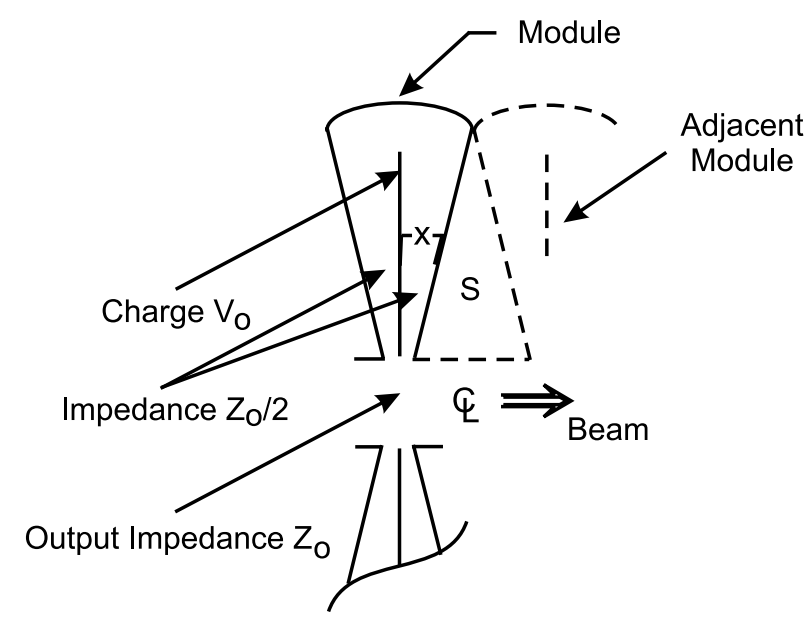

FIG. 6. "Isolated Blumlein" radial pulse line cavity.

the switch drives the same impedances inwards and outwards, the efficiency cannot exceed $50 \%$.

Radial pulse line accelerators that have actually been built (as opposed to just designed) store and switch the energy internal to the induction cavities themselves, as does the isolated Blumlein. The circuits in the cavities are often composed of constant-impedance transmission lines formed from conical conductors like those seen in Fig. 6, in an attempt to make approximately rectangular pulses, in part because the electron beam is easier to transport when its energy is relatively constant on the pulse flattop. If they deliver a simple unipolar pulse, then efficiency must be less than one based on the argument presented earlier. Forming constant impedance conical lines creates shorted regions like $S$ in Fig. 6 which reduce the accelerator inductance and hence the efficiency.

Radial pulse line accelerators are certainly a type of LIA. And in fact radial pulse line accelerators and test cavities can be designed with $E t / I \approx 0.5$ and theoretical efficiencies of $100 \%$. This is possible when the voltage waveform at the gaps in the bore is a bipolar waveform such as those illustrated in Fig. 7, being made up of two pulses (more, in principle) of equal and opposite voltagetime products. The zero voltage-time integral that results means that zero net magnetic flux has been induced in the cavity. Acceleration of the beam takes place on the second part of the pulse and can use all of the available energy.

Zero voltage time integral is a necessary condition to ensure that no net flux and associated energy remain in the cavity after the pulse, but for $100 \%$ efficiency it is also necessary that no flux of any kind (i.e., no equal and opposite flux) exists and that no electric fields remain after the accelerating pulse. An infinite number of transmission line circuits satisfy all these conditions, and five are shown in Figs. 7(a)-7(e). They are shown in circuit schematics; they can be configured from constantimpedance radial lines like those in the isolated Blumlein shown in Fig. 6 or from nested coaxial transmission lines. 
(a)
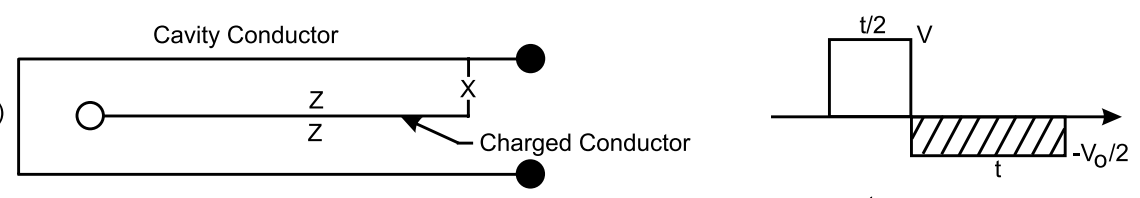

(b)
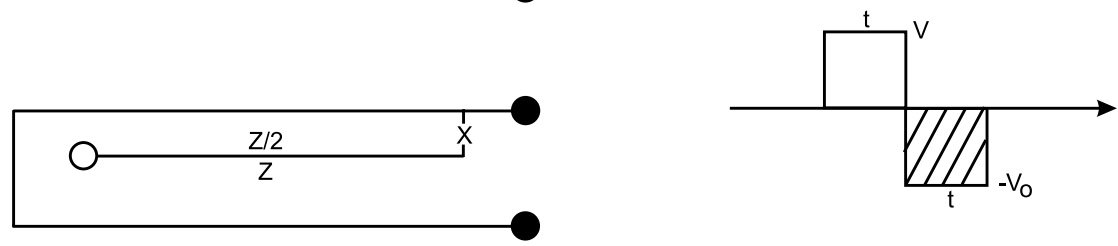

(c)
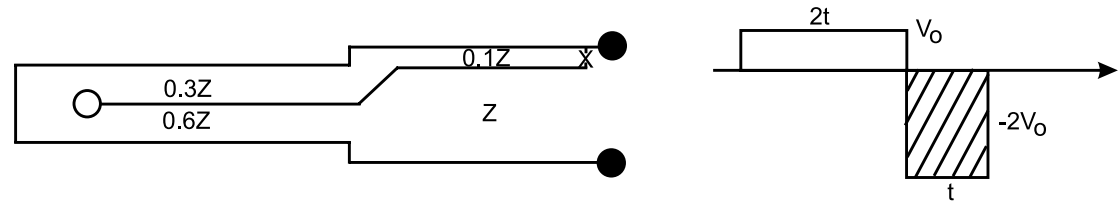

(d)
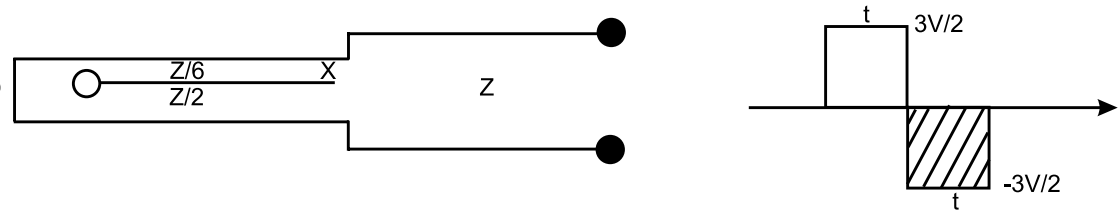

(e)
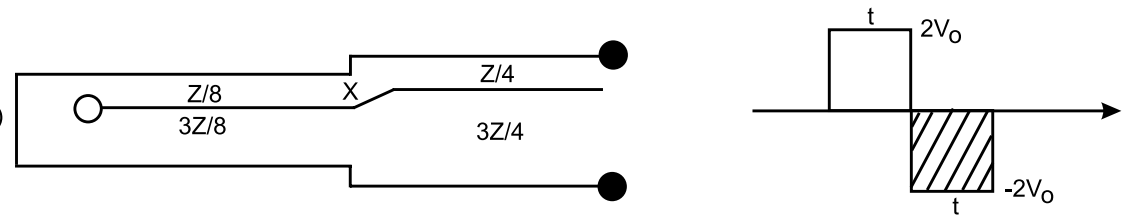

FIG. 7. Air-core bipolar pulse line accelerator cavities. (All have internal conductors charged to a voltage $V$, output impedances $Z$, and beam pulse durations $t$.)

The example in Fig. 7(a) [8] was used in Soviet/Russian accelerators at up to $30 \mathrm{MeV}$ [9] and that in Fig. 7(b) [10] in a test cavity built at Sandia [11].

Figures 7(a) and 7(b) are the first two in an infinite series of designs formed [12] by folding the switched line of a Darlington network about its midpoint to form the cavity, and Fig. 7(c) is the fourth in this series. The designs in this series have matched voltage gains (defined as the accelerating voltage — the second part of the bipolar pulse - divided by the charging voltage) equal to $n / 2$, where $n$ is the number of sections in the Darlington line. As $n$ increases, the first part of the bipolar waveform remains equal in amplitude to the charge voltage and increases in duration, while the accelerating voltage increases in amplitude with fixed duration.

The example in Fig. 7(d) [10] was also built as a test cavity at Sandia, and with Fig. 7(a) it forms the first two of another infinite series of $100 \%$ efficient designs. Each design has just two charged sections; later designs in the series have increasing length, uncharged stepped output sections, and increasing gain with equal duration, equal amplitude, and opposite polarity output pulses [12].

Figure 7(e) [13] shows a circuit from yet another family. It uses all components of a particular Darlington network, the lines of impedance $Z / 8,3 Z / 8$, and $6 Z / 8$ ( $=$ $3 Z / 4$ ) being the sections of the switched line and the section $Z / 4$ being the unswitched line of the Darlington network. Any Darlington network with an even total number of sections is a part of this family. The bipolar waveform components are always of equal magnitude and duration. Reference [13] describes how the design in Fig. 7(e) was used in a $15 \mathrm{MeV}$ accelerator. The injector was based on a $100 \%$ efficient circuit of yet a different type, similar to Fig. 7(e), but with an additional single section of uncharged output line [14].

While the ideal impedance values in Fig. 7 are required to obtain $100 \%$ efficiency, deviations from the ideal values can change the gain with only a second-order reduction of efficiency. In the injector circuit in Refs. [13,14] the matched gain of the ideal circuit is 3 , but the matched gain of the nonideal circuit described is 3.6 , and the efficiency when matched is $90 \%$. In the actual version of the injector [14] the operating (mismatched) gain was 6.4.

As noted earlier, the bipolar circuits of Fig. 7 may be formed either from radial lines (as in Fig. 5) or coaxes (as in Refs. [13,14]). In the coaxial case the length of the pulse line is along the axis of the accelerator, and this may 
lower the accelerating gradient. In the radial case the gradient may be higher but be limited by the expansion of line spacing with a radius that effects constant line impedance. Higher gradients could be achieved in radial line designs by using mixed dielectric (e.g., water and plastic) to achieve constant impedances at fixed transmission line spacing. When the various possibilities are considered, it can be seen that for any given current, maximizing the accelerating gradient corresponds to maximizing the accelerator inductance while obtaining an impedance match to the beam. Inductance is maximized by placing the transmission lines radially and (if mixed dielectrics are used) by eliminating the closed flux-excluded cavity that is used in Fig. 5 to maintain constant impedance.

Circuits exist for radial pulse line accelerator drivers by external pulse generators [15], but to the author's knowledge none has been constructed.

Pulse line modules with monopolar outputs can readily be used in IVAs, as in RADLAC-SMILE [7]. If bipolar pulse lines are to be used in IVAs the situation is more complex. It may be necessary for the IVA bore to withstand the reverse precursor pulse and for the diode to be isolated or nonconducting during the precursor. These conditions are not necessarily hard to meet. An alternative would be to isolate the IVA bore from the precursor with a switch that closes on the main pulse. This was done in Ref. [14], where the use of only one bipolar pulse line module (in an electron injector) made it relatively simple.

Another condition for efficient operation of a bipolar circuit is that the load, which in an IVA is the diode, must be in a specific location with respect to the transmission lines. This condition could be approximately satisfied if the bore has a small electrical transit time. This appears to be the case in some examples given in Ref. [16] of bipolar circuits in a series used to drive diodes that produce microwaves; in other examples in Ref. [16] the bore length appears significant.

It is possible that the condition of placement of the load with respect to the transmission lines could be satisfied by using circuits like those of Fig. 7(d) that have passive output lines, making the bore provide part of this line for each cavity, but this may be too complex to be practical.

\section{E. Multiple pass or recirculating LIAs}

LIAs can be quite long, since they have gradients of the order of $1 \mathrm{MV} / \mathrm{m}$ and can be designed for quite high energies, for example, $50 \mathrm{MeV}$ in Advanced Test Accelerator [17]. An obvious way to reduce the length of a LIA is to pass the beam several times through a reduced number of cells that occupy a correspondingly reduced length. This requires bending the beam and further complicates the design of magnetic guide fields because they must transport particles of different energy through the same cell.

A multipass approach may maintain a constant cell voltage as the beam recirculates, making the design equivalent to a betatron. If ferromagnetic cores are used, the total volt seconds to be withstood by all cores is the same as that in a single-pass LIA that has the same total acceleration. Exchanging length for radius [Figs. 8(a) and $8(b)]$ results in the core weight actually increasing. Thus in this scheme while beam recirculation reduces the length of a LIA, in some ways it makes it less, rather than more, "compact." Folding a single-pass LIA can achieve length reduction without increasing mass, Fig. 8(c).

A more compact recirculating design results if the beam occupies only a part of the circumference of the accelerator, and while the beam is absent from each cell the core of that cell is reset for the accelerating pulse on the next beam pass. In this case the core weight is reduced from that of the LIA by a factor equal to the number of passes. This can result in a truly compact design, Fig. 8(d). However, it poses challenges for the pulse power driver, which must produce a bipolar pulse train. When this scheme was first proposed [18], Birx postulated a "branched magnetics" driver that could produce an indefinitely long pulse train of the type required. Reference [18] describes a design with 75 recirculations of a $10 \mathrm{kA}, 20 \mathrm{~ns}$ beam that is accelerated to $200 \mathrm{MeV}$. The accelerator was termed an induction racetrack accelerator (IRA); Putnam later designated it as an induction synchrotron. Unfortunately, branched magnetics has never been demonstrated except at a benchtop model scale. Arrays of vacuum triodes or of field-effect transistors (FET)s [19] probably represent the nearest term technology for producing very long bipolar pulse trains and driving an induction synchrotron with many beam recirculations.

There are also challenges in beam transport if a single magnetic field system must guide the beam repeatedly around a recirculating accelerator as the beam energy increases over a long pulse-train acceleration. Magnetic fields must be increased during acceleration. Ramping a simple bending field was described in Ref. [20]. Putnam et $a l$. [21] devised and tested at PSI an alternative transport scheme designed for a relatively small number of passes, in which each pass through the cell and the accelerator occurs in a separate beam pipe with complex static magnetic fields, Fig. 9. The overall form of the beam line is a spiral, resulting in the name Spiral Line Induction Accelerator (SLIA). Putnam's team [22] demonstrated a beam transport design that maintained high beam quality for a single-turn SLIA at $10 \mathrm{kA}$, and then for a two-turn SLIA at $2 \mathrm{kA}$, with higher currents not attempted. The $2 \mathrm{kA}$ case used ten ferrite-core induction cells of $330 \mathrm{kV}$ each and attained a total energy of $>9 \mathrm{MeV}$. For convenience, the SLIA demonstration applied a single unipolar pulse to the ten cells used to demonstrate beam transport. 


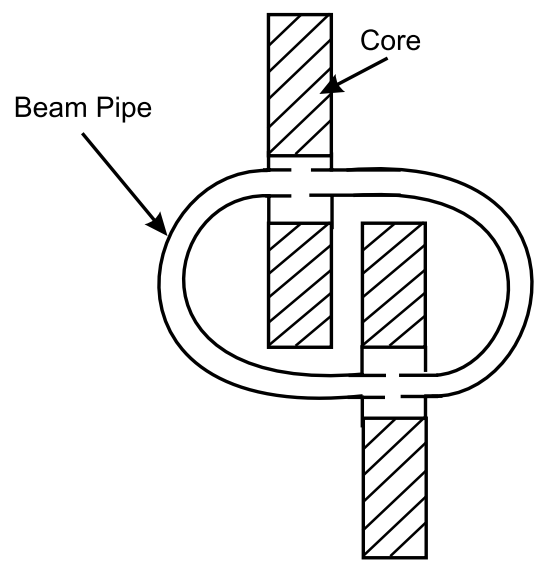

(a) Four passes, two cells.

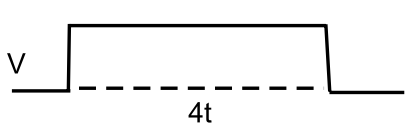

M.

(b) One pass, eight cells.
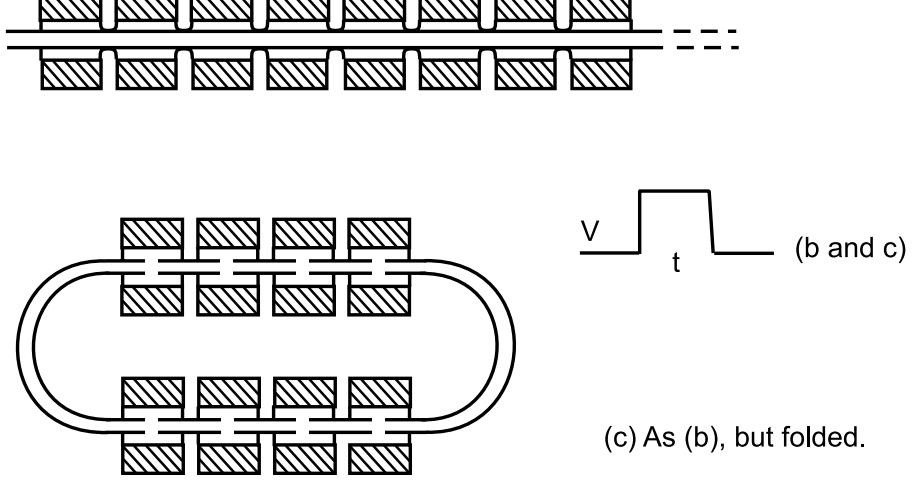

(c) As (b), but folded.

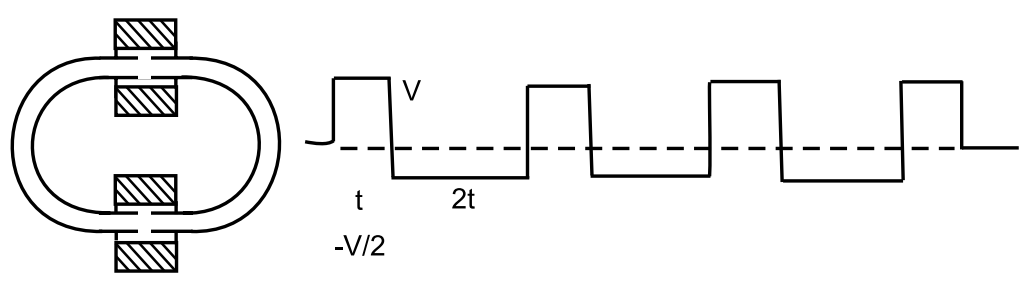

(d) Two cells, four passes with interpass reset.

FIG. 8. Schematic comparison of recirculating and linear accelerators.

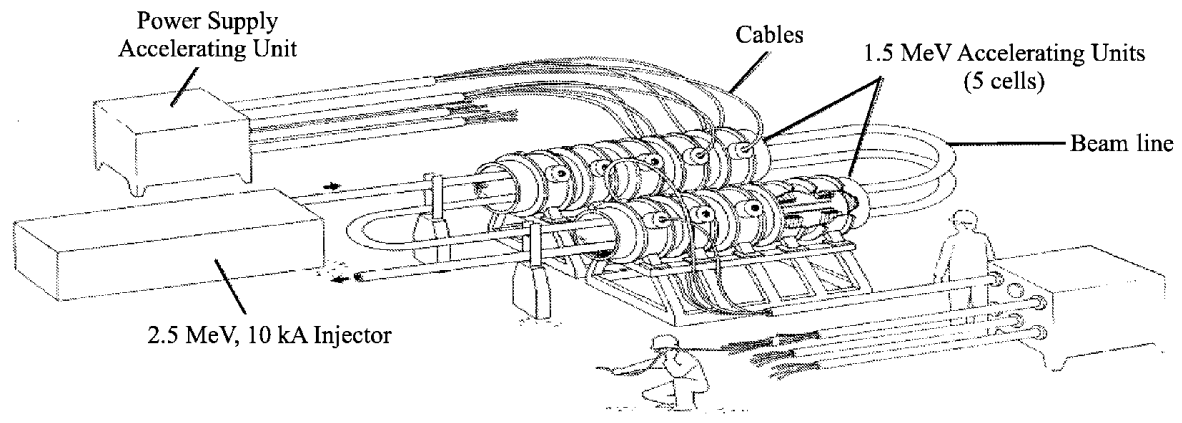

FIG. 9. Spiral line recirculating induction accelerator (two beam passes are shown illustrating actual $9.5 \mathrm{MV}, 2 \mathrm{kA}$ proof of principle). 


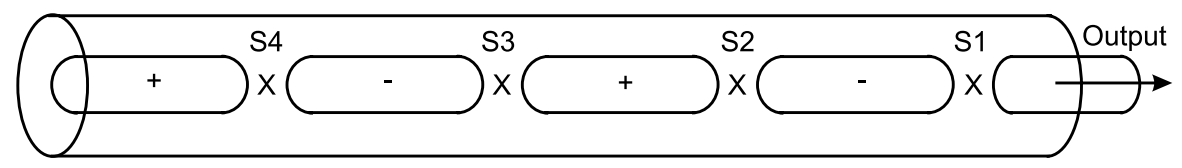

FIG. 10. Frozen wave generator concept for providing short bipolar pulse train. (All switches are closed simultaneously. Variants with sequentially closed switches may be useful.)

For a SLIA, the number of passes is limited by the number of beam pipes that can thread each cell, and for this relatively small number of passes a bipolar pulse train effecting the periodic reset needed in a truly compact design might be effected by a finite pulse train generator such as a frozen wave generator or segmented coaxial line (Fig. 10) rather than by vacuum tubes or FETS.

Note that in the compact bipolar recirculation scheme illustrated in Fig. 9, the use of a ferromagnetic core in the cell could be desirable to reduce the parasitic cell current on each pulse, but the resistive behavior of the core results in it dissipating much of the energy delivered to it on each pulse. On the other hand if the cell is air cored (has no ferromagnetic core) it will receive more parasitic current but the energy need not be dissipated and would not increase on successive pulses because of the bipolar nature of the pulse train. This is illustrated in the possible recirculating LIA shown in very simple form in Fig. 11.

The accelerator consists only of a beam pipe that has two parallel straight lengths rounded at the ends by $180^{\circ}$ bends. A symmetrical bipolar waveform, idealized in Fig. 10, is applied to the gap in the center of one length of the beam pipe, which is assumed to be in a medium

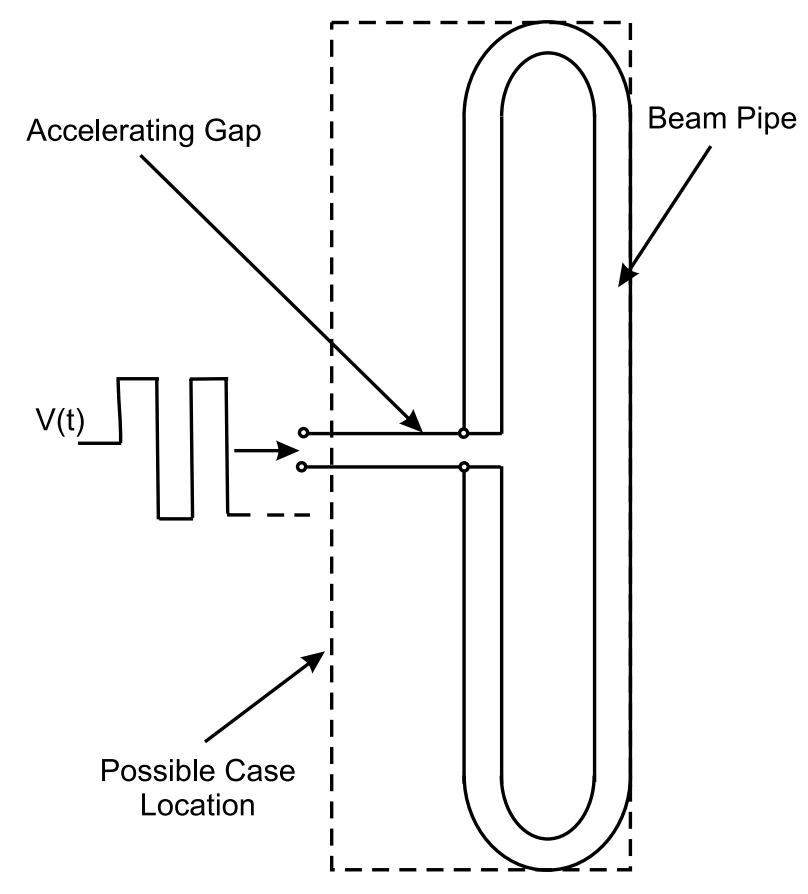

FIG. 11. Air-core recirculator without cells. with permittivity one (vacuum, air, or $\mathrm{SF}_{6}$ ). The beam pipe presents to the pulse driver a transmission line parasitic load. The beam occupies half the pipe perimeter, and at the end of the beam pulse a reverse reflection of the accelerating pulse arrives from the short circuit formed by the bends at the ends of the beam pipe to provide, or to help provide, the reverse voltage portion of the waveform that continues until the beam head reappears.

Injection and extraction are not considered. To estimate parasitic currents, we take the pipe separation to be five pipe diameters. Depending on whether the continuous side of the beam pipe is in a cylindrical shell surrounding the side with the gap (i.e., the case in Fig. 11 is present) or is an isolated pipe (the case is absent, and the electromagnetic fields are not contained), the transmission line impedance seen by the pulser is $\sim 50 \Omega$ or $\sim 150 \Omega$. For $600 \mathrm{kV}$ acceleration per turn (for example) the parasitic pipe current is 12 or $4 \mathrm{kA}$. In this case it is not necessary for high efficiency that the beam current be large compared with these values. As the beam energy builds up on successive turns the energy stored in the beam pipe inductance remains limited to a maximum value, because the waveform applied to the pipe has zero time integral. It is simply necessary that the drive circuit, the details of which are not considered here, be able to drive the beam and the pipe in parallel with the correct waveform.

The beam pipe would have more inductance if it formed an open circle. In this case (unless it was enclosed in a torus) it would have no transmission line properties and would radiate electromagnetic interference.

Note that the beam pulse duration has not been specified here; it determines the accelerator length $(1 \mathrm{ft}, 30 \mathrm{~cm}$, total per ns) but does not affect the calculation of parasitic current.

Another approach to recirculating acceleration over a limited number of passes was also explored on paper by the author (following a suggestion by Eccleshall [23]) in a $200 \mathrm{MeV}, 10 \mathrm{kA}, 20 \mathrm{~ns}$ design in Ref. [18] and was also later developed experimentally by Sandia [11]. In this approach the cell cavities are bipolar pulse lines of the type illustrated in Fig. 6, but their matched currents are much greater than the beam current instead of being equal to it. The cavities therefore ring, producing damped pulse trains in which forward accelerating pulses are separated by reversals that can be regarded as "resetting" the air-core cavities in the same way as cores would be reset by reverse voltage pulses in the ferromagnetic cavities of recirculating accelerators. In the example in 
Ref. [18], the drivers are 43 coaxial versions of the circuit in Fig. 7(b), each charged to $0.46 \mathrm{MV}$ and arranged to approximate a circle, with the beam passing through each coax roughly on axis.

One disadvantage of the ringing air-core approach is the fact that the switched currents must be much higher than the beam. The switch inductance then causes pulse train waveforms to have long rise times and exacerbates another problem, the tendency of the waveform to deteriorate progressively from the ideal rectangular form on successive acceleration pulses in the pulse train. Another disadvantage is the decreasing cavity voltages on successive accelerating pulses; in Ref. [18] this was mitigated by progressively switching in modules, then after their energy had been extracted over eight cycles crowbarring them to avoid their decelerating the beam. The relatively smooth acceleration obtained in this way (as with the branched magnetics-driven IRA) allowed a guide field system to be ramped steadily over the acceleration, bending the beam in a circle into which the coaxial drivers fitted.

We note that the recirculating bipolar accelerator designs of Refs. $[11,18]$ used circuits like those in Fig. 7 that produce waveforms that start with a reverse polarity. For multipass acceleration this may not be necessary, and other circuits may be better than the radial pulse line circuits that were originally devised to give all their energy on a single pulse or cycle.

\section{THE INDUCTION VOLTAGE ADDER}

\section{A. The advantages and history of the IVA}

In Sec. III we shall describe the principles of the design and construction of IVAs in more detail. The IVAs we are considering are substantial pulse power machines, delivering more than $2 \mathrm{MV}$ (up to $20 \mathrm{MV}$ ) and more than $50 \mathrm{kA}$ (up to $750 \mathrm{kA}$ ) for pulse durations of tens of nanoseconds. Unlike the 2-10 kA IVAs that form the injectors of typical LIAs, all the examples we shall consider use Metglas-type ferromagnetic cores rather than the ferrite used in LIAs, because their large currents allow them to tolerate larger loss currents. Again because of the larger currents, in most cases they use magnetic insulation in vacuum rather than operating below the threshold fields for electron emission and breakdown, which would result in too much inductance in the vacuum. We begin by discussing the advantages of IVAs.

One of the main advantages of the IVA concerns not the adder itself but the energy density of the pulse forming lines (pfls) that drive it. The IVAs built in the USA have load impedances in the range 25-150 $\Omega$. The alternative to adding pulse generator sources in an IVA to produce pulses of tens of ns duration is to use a single multimegavolt source with an impedance that roughly matches this load impedance range, and the best such source is the coaxial oil dielectric pfl. Many of these have been built, especially before the development of IVAs. But because of its permittivity $\varepsilon=2.3$, the energy density $\left(\varepsilon F^{2} / 2\right)$ of oil is less than a tenth of that of water, which has $\varepsilon=80$ and an operational electric field $F$ that is not much lower than that of oil. Because of the high permittivity, coaxial water lines have lower impedances than oil lines, in the range of a few ohms to $10 \mathrm{ohms}$. The IVA, by using pfls in series, allows the individual $\mathrm{pfl}$ impedances to be in this range and still match the $25-150 \Omega$ loads, and thus makes the use of water possible. This greatly reduces the volume of liquid dielectric needed to generate the pulse power. In addition, the insulation volume required by the generators (usually Marxes) that charge the pfls is less at the lower charge voltages. The cost of dielectric and insulation is further reduced because water is cheaper than oil, and oil must be handled more carefully to avoid spills, especially in large volumes.

Another advantage for IVAs arises if the load current rise time is limited by the vacuum insulator stack. Solidinsulator vacuum interfaces have the lowest dielectric strength encountered anywhere in most pulse power machines, because of the speed with which low density regions, which have very low breakdown fields, can form near the interface. Because it must therefore be long, the insulator stack has significant inductance. As the stack length increases with voltage, the width must increase in proportion, as illustrated in Fig. 12, in order to grade the insulator voltage; the inductance, which is proportional to the insulator's cross section area, therefore increases as the square of the voltage. The sum of the inductances of lower voltage stacks increases only linearly with voltage, proportional to the number of stacks, and therefore the total stack inductance in an IVA will be lower than that of a full voltage stack.

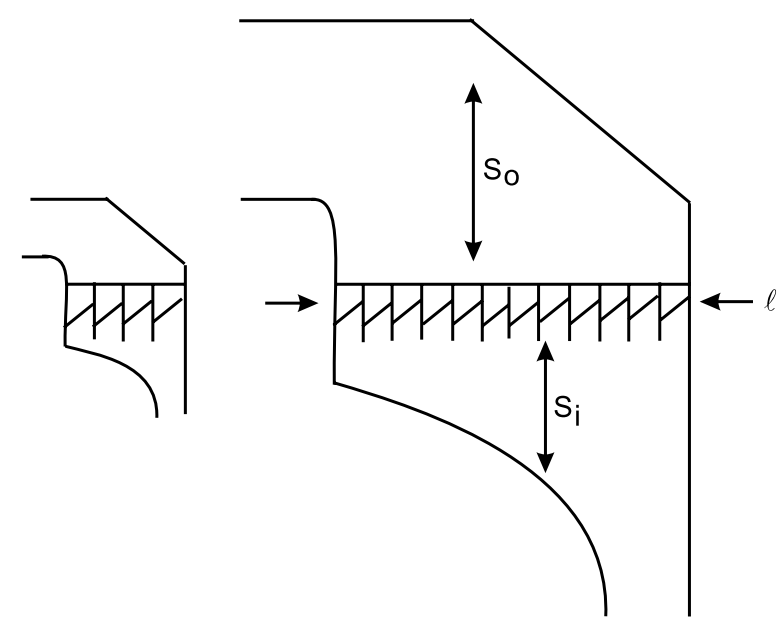

- Length $\ell \propto \vee$ for flashover.

- Internal width $S_{i} \propto V$ for grading and for fixed field allowed on gradient ring tips. - External width $S_{O} \propto V$ for grading.

FIG. 12. Inductance of insulator stack of fixed radius scales as $V^{2}$. 
Another potentially important advantage of the IVA is that the energy stored in each pfl can be relatively small, first because the energy is divided between a number of pfls in a series. Secondly, further division in parallel is made easier because all pfls are effectively at ground potential and have relatively low voltage, so they can readily be switched synchronously in parallel. A small energy per module reduces the damage in a fault, and this can help reliability, especially in repetitive systems.

The last advantage, the use of small modules to achieve high reliability, was in fact what motivated the suggestion of the IVA approach for big pulse power machines in 1979 [24]. The development issues that the then-new IVA approach posed were principally associated with the high currents (and $d i / d t$ ) compared with the IVAs already in use as LIA injectors and the much lower inductance adders that this required. The main innovation proposed in the IVA in order to reduce inductance was the use of magnetic insulation to allow the vacuum regions to operate at much higher electric fields than those of the LIA injectors. In 1979, magnetic insulation had been used for some time to propagate power in vacuum many meters at a high output voltage, first in Aurora [25]. The novel feature of the magnetic insulation needed in a voltage adder was its application in a configuration where the voltage and impedance increased stage by stage in vacuum. This and other features of adder design, especially the cells, required a "proof-of-principle" demonstration, and this was made on Helia [26] in 1985. Achieving lower inductance in an IVA was also helped by using, instead of the ferrite cores used in IVA injectors, the Metglas cores that were just becoming available. Metglas has a much higher flux density swing, $>3 \mathrm{~T}$, compared with $\geq 0.5 \mathrm{~T}$ for ferrite, and this shortened the adder and reduced its inductance. The use of Metglas-core cavities under short megavolt pulses was also first demonstrated in Helia.

In the following sections dealing with the design of various regions of IVAs we begin with the most key region, the vacuum bore, where magnetic insulation and voltage addition occur and where effective inductance and losses must be minimized. We then proceed to discuss the cells in detail. We finally make some general comments on the pulse power needed for IVAs.

\section{B. Design of the adder bore}

In this section we describe the principles used to design the adder bore, the cylindrical region that threads the induction cells of the IVA. We first describe how this can be designed as a series of transmission lines of appropriate impedance. Then we discuss how magnetic insulation is achieved in a way that provides the desired impedances. We then discuss alternative insulation schemes for the adder bore.

\section{Impedance matching}

The bore of an IVA often has an electrical transit time comparable to, or even longer than, the pulse duration, so that in order to couple to the load efficiently and with fast rise time it must behave like a transmission line system approximately matched to the drivers or to the load.

(a)

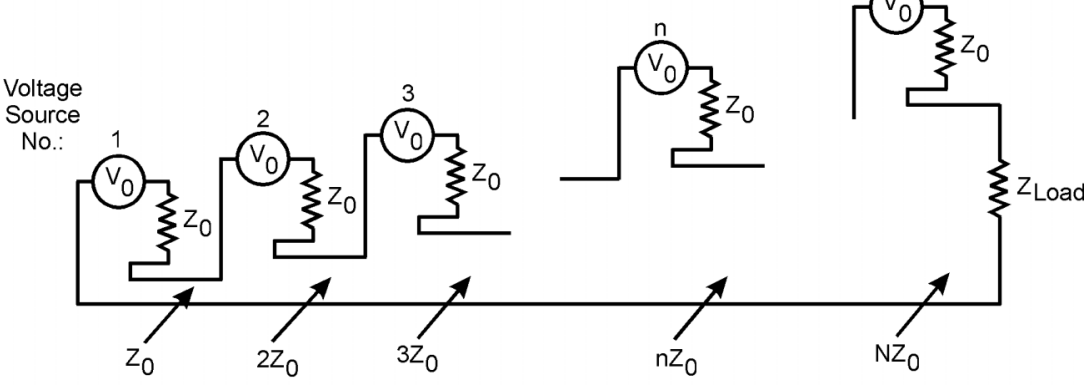

Wave Impedance of Transmission Line Sections

(b)

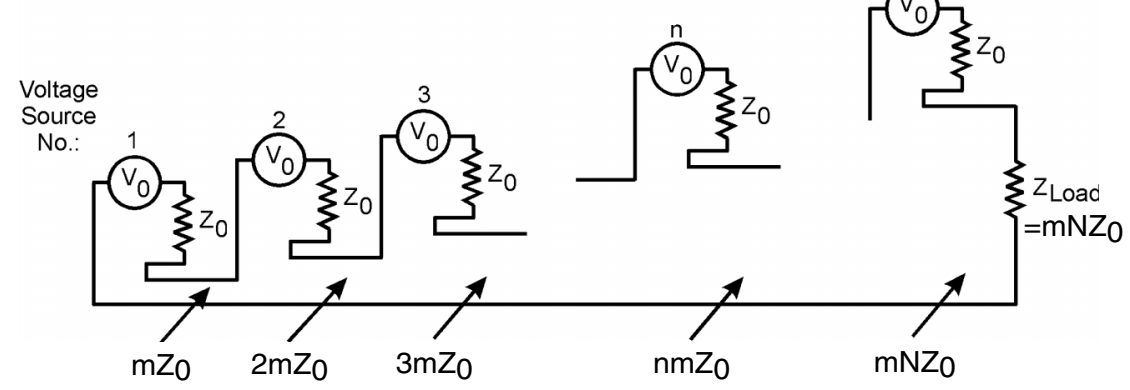

FIG. 13. Electrical schematic of (a) driver-matched and (b) load-matched transmission line voltage adders. 
The scheme normally used is that of the transmission line adder illustrated in Fig. 13(a) which was first devised by the author for the design of the injector of the FXR LIA [27] at Lawrence Livermore National Laboratory in 1977. The drivers, assumed to be identical, are timed so that their pulses are simultaneous at the load. The adder impedance is matched to that of the individual drivers, $Z_{o}$, by giving each of the $N$ adder sections a wave impedance $n Z_{o}$, where $n$ is the number of drivers upstream. It can readily be seen that this results in all adder sections being matched looking upstream. The voltage at an arbitrary-impedance load must therefore immediately rise to, and stay at, the voltage that the drivers would produce if they were connected in series to it without an adder in between; because any pulse moving upstream (e.g., a reflection from the load or a pulse sent upstream within the adder during injection from the drivers) is matched at all line junctions and cannot therefore reflect back towards the load. In fact, no net pulse is sent upstream from the drivers.

It is important to note that each driver looks into the adder and (with the exception of the driver farthest from the load) sees a impedance higher than its own, formed by the adder sections upstream and downstream, in series. By itself, the driver would therefore develop a voltage that may approach its open circuit voltage and would send a pulse in both directions in the adder. But the pulse arriving from upstream at the same time brings this driver voltage to its matched value, and the reflection of the pulse from upstream exactly cancels the pulse that the driver would send upstream. Thus the full matched power of all drivers is smoothly transmitted downstream and eventually arrives at the load; if the load is roughly (or exactly) matched to the adder output impedance $N Z_{o}$ it will develop nearly (or exactly) the total matched driver power. If it is mismatched the power will be lower and a significant reflection from the load into the adder will occur, but the reflections do not return to the load until they have been reflected within the drivers.

It is also possible to design an adder that is matched to the load rather than to the drivers. Figure 13(b) shows this; each impedance of the adder sections in Fig. 13(a) has been multiplied by a factor $m$ to become $m n Z_{o}$, and the load impedance is no longer arbitrary but is $m N Z_{o}$, matching the adder output impedance. In this case also the adder couples the drivers to the load as if the adder sections were absent-immediately and with no pulse distortion. It is not possible to prove this by simple statements, as we did earlier for the driver-matched adder, and a mathematical proof needs more space than is available here.

The driver-matched adder has been used in IVAs in practice, perhaps because the existence of the loadmatched adder was not recognized until more recently, in the design of RITS [28]. The driver-matched adder has the advantage that it "works" perfectly for any load impedance, not just a matching load. But as we shall see in the next subsection, a magnetically insulated adder often necessarily has a matching load. The load-matched adder may therefore have no disadvantage if it uses magnetic insulation. It has the advantage that it allows a mismatch between load and drivers, and this flexibility may be useful whatever type of bore insulation is used.

\section{Magnetic insulation-the negative IVA}

Magnetic insulation is especially appropriate to design the vacuum bores of IVAs when the inner conductor threading the cells and driving the diode (the "stalk") is negative ("negative adder"), and this is the case chiefly considered in this subsection. It may also be appropriate when the stalk polarity is positive ("positive adder"), particularly if the adder is electrically short compared with the pulse.

The process of designing a magnetically insulated negative adder consists mainly of choosing an appropriate radius in each cell for the inner conductor (the stalk) that threads the bore and delivers voltage to the load. The stalk radius in each cell is chosen in conjunction with the bore radius (which in IVA designs to date has for convenience of construction been constant through the adder) so that the stalk impedance in that cell is the value required by the discussion in the previous Sec. IIIB1. In the drivermatched adder (see Sec. IIIB1), the impedance in the $n$th cell is made to be $n$ times the driver impedance $Z_{o}$. The impedance $n Z_{o}$ is given by $V / I$ where $V=(n / N) V_{\text {load }}$ is the voltage in the $n$th cell of an $N$ cell adder and $I$ is the current, which is the same in all cells. The design radius chosen for the stalk is the one that would give an operating impedance $Z_{\mathrm{op}}$ that is equal to the desired value $n Z_{o}$ in a long magnetically insulated coax.

In such a long magnetically insulated coax, or in general for magnetically insulated transmission lines (MITL) [29], a voltage wave propagates at a velocity somewhat less than that of light. At the wave front, some electrons cross the gap from the negative conductor (here the inner) to the positive (outer) conductor, but behind this "loss front" a stable magnetically insulated regime exists without any losses. This regime has the total return current $I$ flowing in the positive conductor, but only a fraction of this current, the "bound" current, flows in the negative conductor, while the remainder of the total current flows in the form of an electron sheath outside it. In the case of a coax with a negative inner conductor, this sheath surrounds the inner conductor.

Although the loss-front electrons represent a fairly small energy loss, their effect on IVA cell components may need to be considered. A method of suppressing loss-front electrons by applying a small reverse voltage (a "bias" voltage insufficient to produce electrons) to the center conductor before the pulse, increasing the displacement current in the pulse wave to the value needed for 
insulation behind the wavefront, was suggested in studies of the 30 MV LIBRA IVA design described in Ref. [30].

The operating impedances and other information about the magnetically insulated region in an unbiased MITL are found in Ref. [31]. The usual operating impedance $Z_{\mathrm{op}}$ of a coax is less than that of the vacuum wave impedance $\left(60 \ln \left(r_{o} / r_{i}\right)\right.$, where $r_{o}$ and $r_{i}$ are the inner and outer radii) by a factor whose dependence on the voltage $V$ is shown in Fig. 14(a). Knowing the voltage $V$ at any IVA cell and

(a)

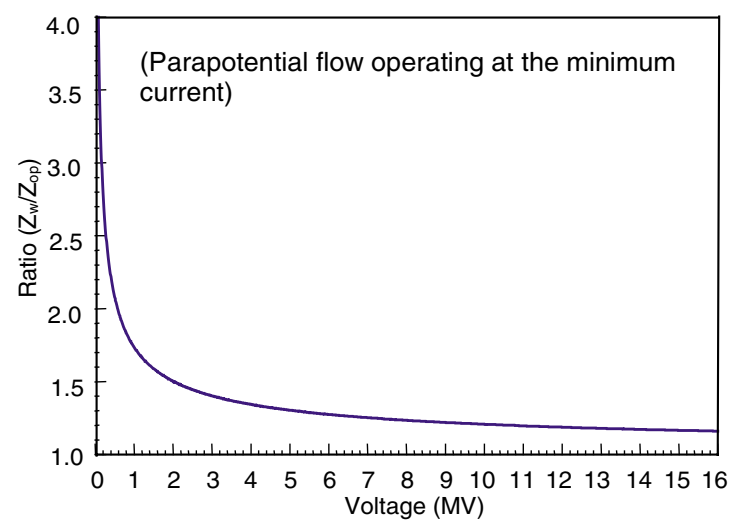

(b)
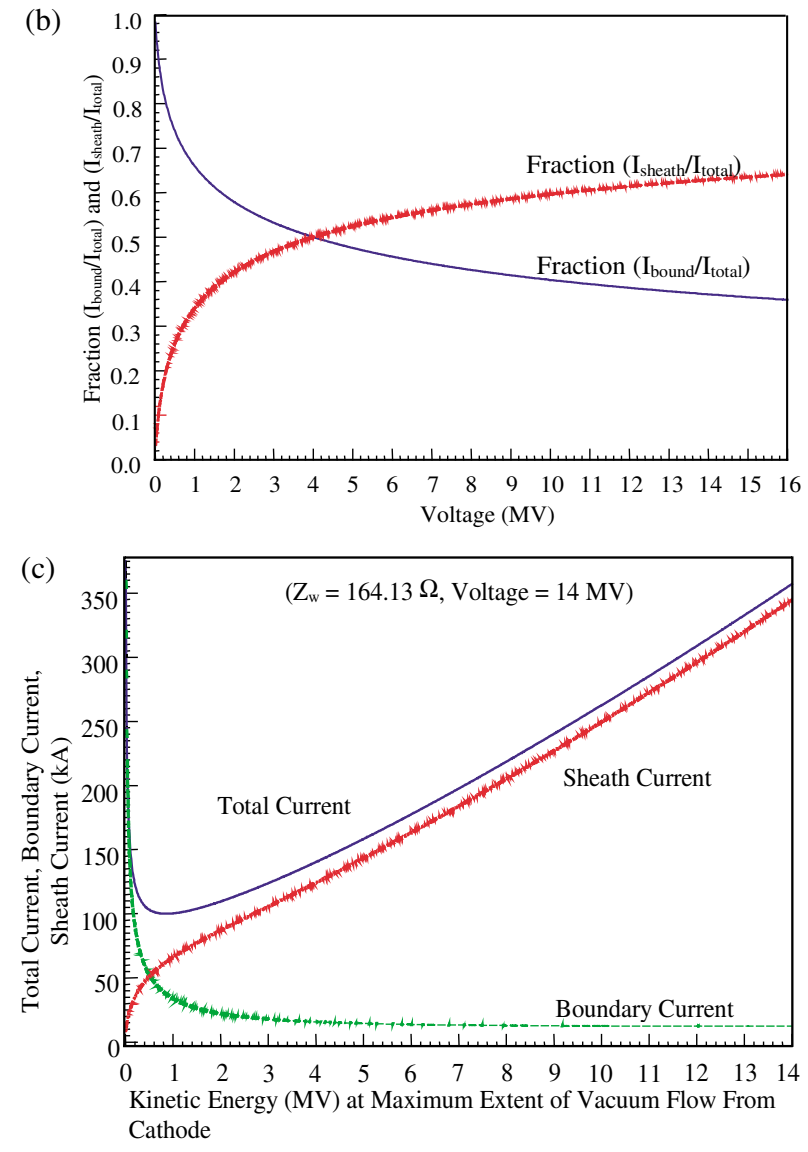

FIG. 14. (Color) Magnetic insulation parameters as a function of voltage: (a) ratio of vacuum impedance to operating impedance of a MITL; (b) fractions of bound and sheath current in a MITL; (c) range of operating currents in a MITL [31]. the current $I$, the stalk radius can be chosen using Fig. 14(a) to make the operating impedance $Z_{\text {op }}$ equal to $V / I$.

Figure 14(b) shows the fractions of current that are bound and sheath as a function of voltage, showing that the electron sheath current fraction increases with voltage. For a given impedance coax at a given voltage there is in fact a range of possible operating currents, each with two possible divisions between bound and sheath current, as illustrated in Fig. 14(c). The minimum possible current $\left(I_{\min }\right)$ seen in the figure is usually found experimentally to be the actual current in a long magnetically insulated coax (i.e., one that is not yet affected by its downstream load or by upstream features that inject more sheath current), and this corresponds to $Z_{\mathrm{op}}$ and to the conditions represented in Figs. 14(a) and 14(b).

In this way magnetic insulation theory defines an ideal set of radii for the stalk in each cell. The stalk may be built of sections having the ideal radius in each cell, with a step-down in radius at the points in successive cells where the voltage and impedance increase. This is illustrated in Fig. 15, where the step in the inner conductor is shown opposite the radial feed. In this region, it is expected that the inner conductor will emit additional electrons into the electron sheath, as required by the increase of voltage and hence of sheath flow downstream of the step. The geometry of the step on the inner conductor and of the spacing and inner corners of the radial feed will affect electron flow in the region, and could, for example, cause the additional electron current injected into the vacuum to be greater than the differences that correspond to the $I_{\min }$ operation, resulting in more sheath current and operation to the right of the minimum in Fig. 14(c). This would be undesirable because the operating impedance downstream from the junction would be lower than the ideal matched value and because the bound current will be reduced. As we will see later in this section, for some

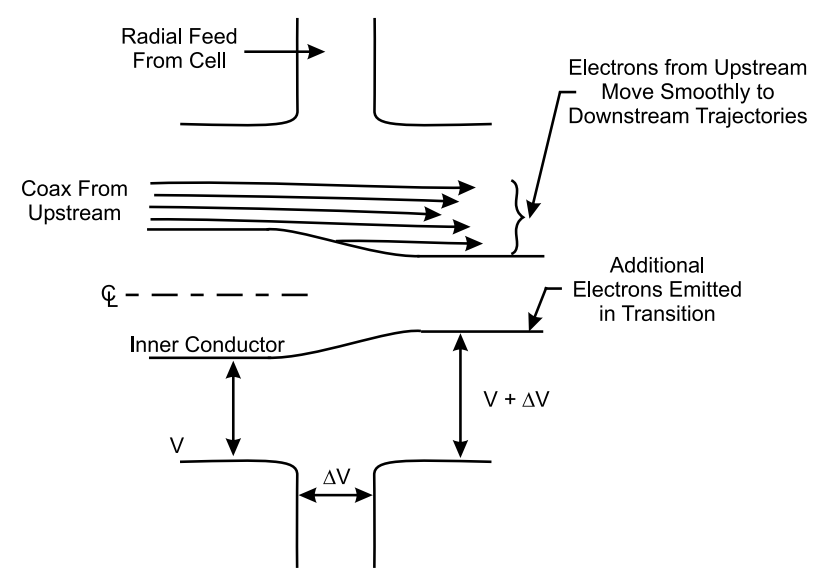

FIG. 15. Schematic of conductor and electric flow in "ideal" voltage addition. 
IVA loads, it may be considered that only the bound current is useful.

There is probably some ideal geometry of the region of the step and the radial feed, principally the shape of the step on the stalk, that maintains a smooth electron flow at $I_{\min }$. However, this geometry has not been explored; it would be expensive to implement it in practice, because all junctions in the adder, being at different voltages, would probably need different geometries. Instead, a smooth step is usually introduced in the stalk over a distance comparable to the radial feed gap or to the inner-outer spacing, and in this way satisfactory results have been obtained.

An alternative to the stepped stalk shown in Fig. 15 is a smoothly tapered stalk that has the ideal calculated radius at some point within each cell. LSP [32] particle-incell code (PIC) simulations by Bailey, Pulse Sciences, of a 15 MV, 150 kA RITS-like [28] stalk are shown in Fig. 16 that compare a stepped design with a tapered design that has the ideal radius at or just beyond each radial feed. The output voltages, the total currents, and the current in the electron sheaths (which are shown in blue in Fig. 16) as a function of time are shown in Fig. 17. The tapered stalk produces less sheath current and delivers more bound current; this may be the result of its slightly higher average wave impedance, but it may also be due at least in part to the injection of extra electrons into the vacuum at each of the steps in the stepped stalk. (Note that even the small zoning steps seen in the tapered stalk inject some extra electrons.) As noted, for some loads it is only the bound current that is useful, and for this reason the tapered stalk appears preferable to the stepped stalk. It may also be easier to construct, having fewer sections.

One might consider making a tapered stalk follow the average contour of the ideal stalk, having the ideal radius midway between radial voltage feeds. But the $I_{\min }$ for the stalk just after each radial feed would then be higher than the desired operating current. LSP simulations confirm the expectation that this region will control the power flow, resulting in lower than desired impedance and more vacuum electron flow.

Note that in the simulation in Fig. 16 the last cell of the adder is followed by a longer section of constant diameter stalk and constant diameter outer. An output MITL of this type is often added to an IVA in order to place the load diode at a convenient distance. If matched to the load this MITL can be very long, since it will propagate the magnetically insulated pulse without loss except for an erosion of the front by loss electrons; at very high voltages $(\sim 10 \mathrm{MV})$ the erosion rate is small. An output MITL can also taper or flare in diameter without loss, provided this is done gradually over some distance, and it can bend, as in Aurora [25].

In the simulations shown in Fig. 16, the adder load is a large area diode, in which it is desired that electron current flow to the end plate to make bremsstrahlung (a)

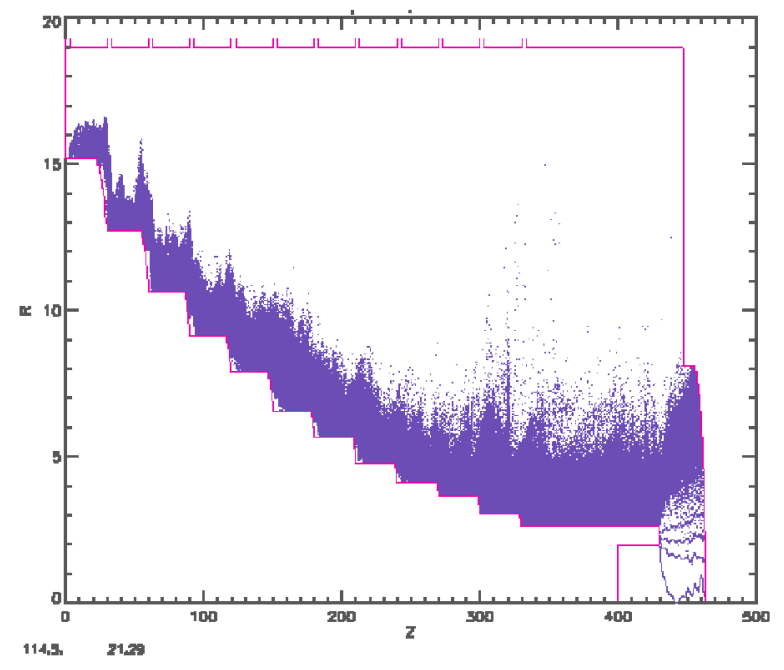

(b)

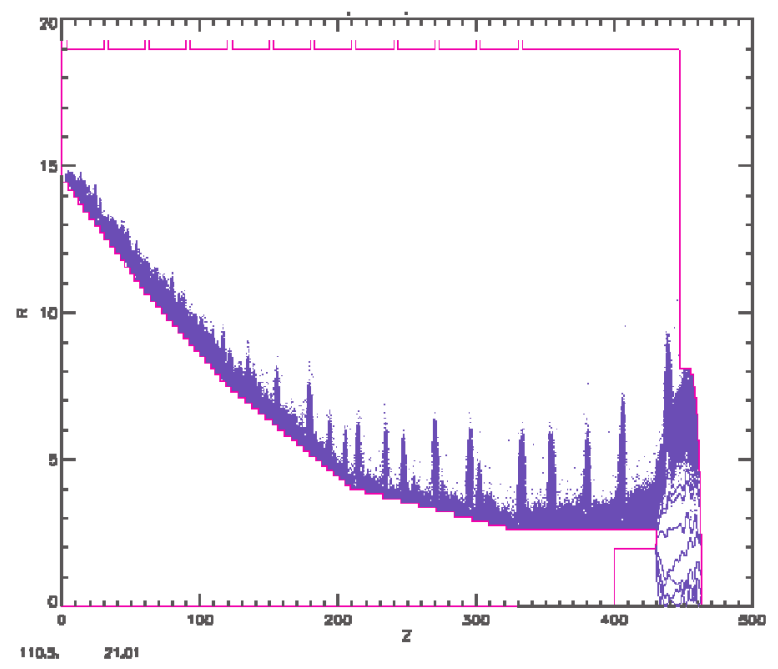

FIG. 16. (Color) LSP simulations of (a) stepped RITS stalk and (b) tapered RITS stalk.

$\mathrm{x}$ rays there. Both the sheath current and the bound current are useful in such a diode, whether the electrons make $\mathrm{x}$ rays at the end plate or pass through it to be used in other ways, because the sheath current travels to the end plate and the bound current is emitted from the end of the cathode to the end plate. For such diodes the use of an IVA is therefore very efficient. This is not necessarily true for other diodes. The immersed $B_{z}$ diode [33] is an example of a diode that may be considered to use only the bound current, while the sheath current flows to ground, Fig. 18(a). In the AWE (formerly Atomic Weapons Establishment, UK) approach to driving a focused paraxial diode [34], the emitting cathode is mounted on a "knob" on the end of the cathode stalk that is large enough to reduce its surface electric fields below electron emission thresholds, Fig. 18(b). Here again it has been thought that the emitter may be able to deliver only the 

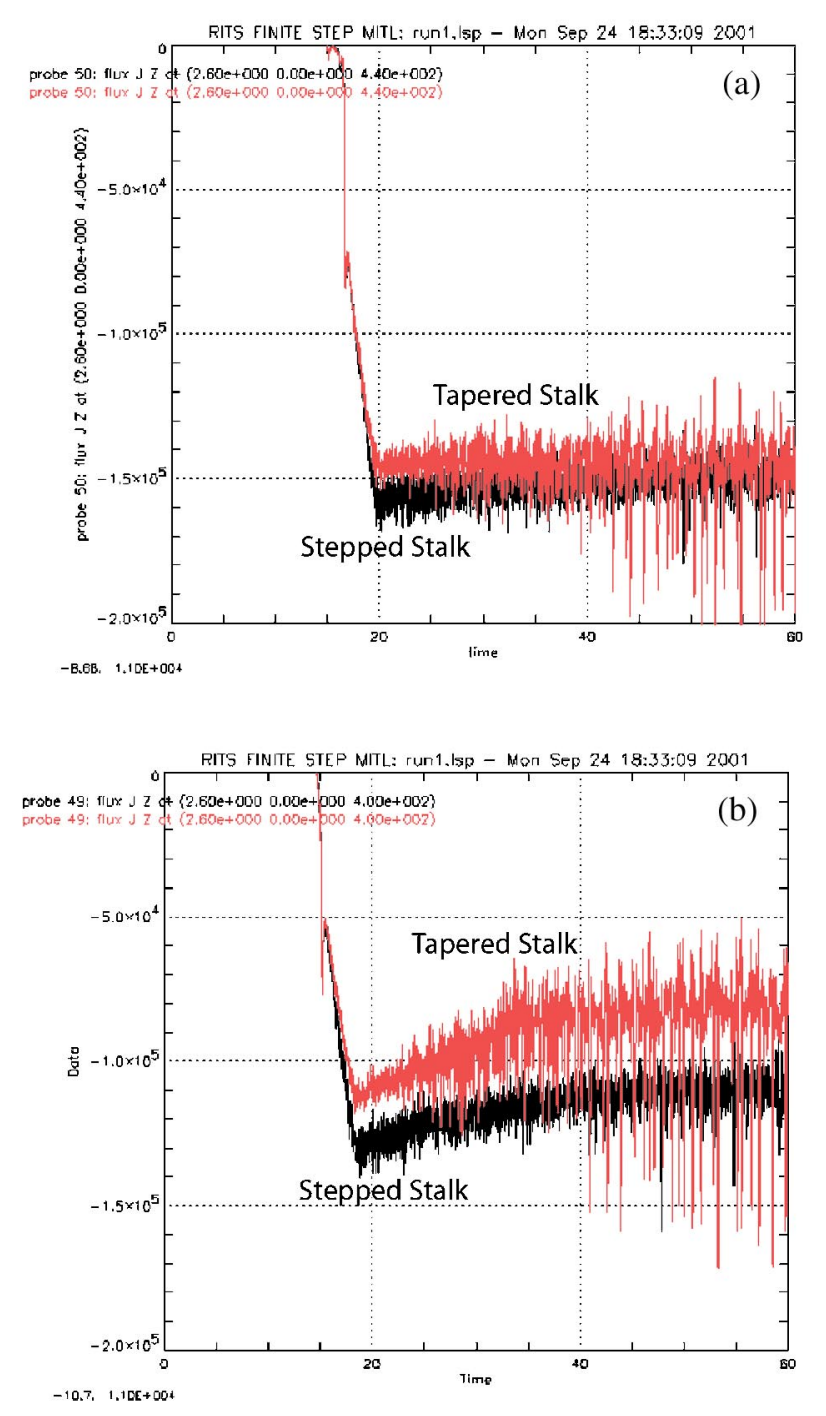

FIG. 17. (Color) Output of stepped and tapered RITS adders (a) total current, (b) sheath current.

bound current in the drive MITL and sheath electrons go to ground upstream of the knob. However, recent PIC simulations [35] suggest that this geometry can "retrap" some of the sheath current and reemit it from the cathode of the paraxial diode, if this diode can be made low enough in impedance. This may also be true in principle of the immersed diode, though reducing the diode impedance far enough to retrap while keeping the diode impedance stable may be difficult.

One way to increase the bound current at the load of an IVA, or of a magnetically insulated line in general, is to design its impedance to be higher than that of the load. (This may affect the impedance and voltage chosen for the drivers if it is desired to operate with a driver-matched adder in order to preserve driver wave shape at the load.) At the load, the magnetic insulation wave then sees an impedance lower than the operating impedance of the last adder section. The load voltage becomes less than that of the magnetic insulation wave that arrives and the total

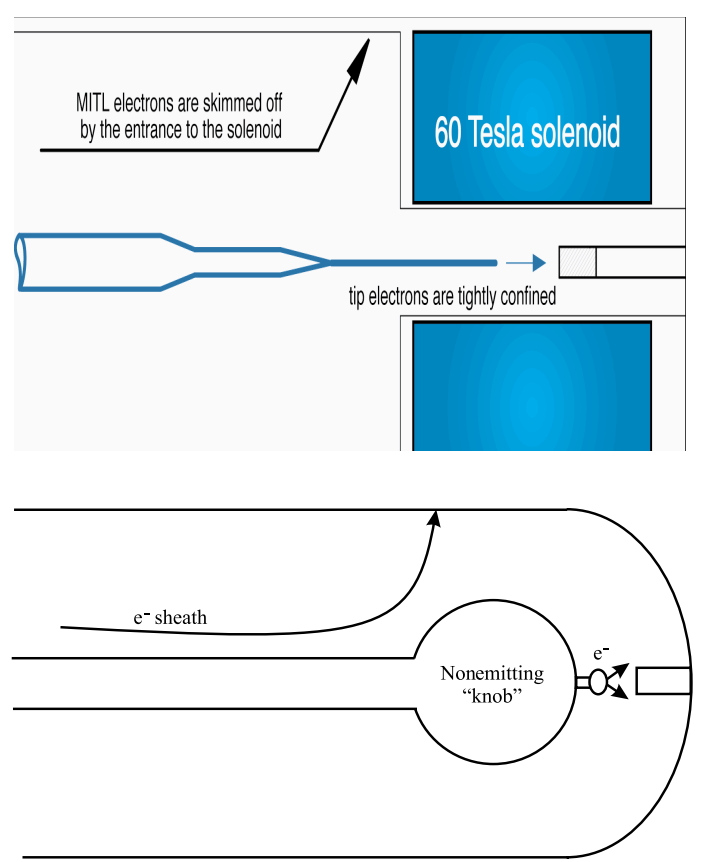

FIG. 18. (Color) Diodes on the end of MITLs. (a) Immersed $B_{z}$ diode. (b) AWE paraxial diode.

current higher. The reflection into the MITL in effect moves the operating point of the MITL from the current minimum seen in the full line in Fig. 14(c), to the left. As can be gauged by examining Fig. 14(c), a small increase in total current and a proportionately small decrease in voltage are accompanied by a large increase in boundary current and reduction in sheath current. The driver voltage must be increased to compensate the reduction in voltage at the diode and the driver power will need to be increased to compensate for the reduction in power due to the mismatch, though the power reduction can be small.

In general, the efficiency of a magnetically insulated negative IVA may depend on whether the sheath flow can be used in the load diode and, if it cannot, on whether the diode impedance is low enough to retrap sheath flow. Independent of sheath flow considerations, the efficiency depends on the impedance of the load diode because the adder cannot in practice have an operating impedance higher than about $150 \Omega$. If it did, the inner stalk would become too thin structurally (unless the adder is vertical and the stalk hangs, or, if horizontal, the stalk is in place only transiently). A load such as the immersed $B$ diode that has an impedance of hundreds of ohms therefore cannot couple more than a fraction of the adder current, and the remainder will flow as electrons to ground outside the diode. Similarly, if a paraxial diode is able to focus only a limited current like $30-40 \mathrm{kA}$, then if it is driven by a $>10 \mathrm{MV}$ IVA with $>10 \mathrm{MV} / 150 \Omega \vee 70 \mathrm{kA}$, half the current or more will flow to ground outside the diode.

It is also possible for conditions near the load or at a transition in a MITL or an IVA adder to cause the 
downstream part of the MITL to operate to the right of the $I_{\min }$ point in Fig. 14(c), with less bound current than at $I_{\min }$. For example, consider either of the transitions in Fig. 19. In Fig. 19(a), the MITL impedance increases at the transition shown. If the downstream MITL operated at $I_{\min }$, the upstream MITL would not have enough current to be magnetically insulated, i.e., to operate at any point on a diagram like that of Fig. 14(c). Electrons might therefore flow to ground at the transition. However, it seems more common that the downstream MITL operates to the right of $I_{\min }$ in Fig. 14, with enough current to insulate the upstream section, and with its extra electron current arriving from upstream and forming part of a larger sheath current that flows on downstream and reaches ground only at the diode.

In the transition shown in Fig. 19(b), the wave impedance is kept constant through the transition. But if this is not done very gradually the effect is again additional sheath flow in the downstream MITL.

In both of the illustrations in Fig. 19, the load is shown as a large area diode that can use the sheath flow as well as the bound current. For diodes that cannot, transitions that increase the sheath current and decrease the bound current should be avoided.

A more detailed magnetic insulation theory exists describing electromagnetic and electron power flow in MITLs. Among the important results [36] is the ability to estimate closely the instantaneous voltage in a MITL of uniform cross section from the instantaneous currents in the positive $\left(I_{A}\right)$ and negative $\left(I_{C}\right)$ conductors and the electromagnetic wave impedance $Z$ :

$$
\begin{aligned}
V \cong & Z\left(I_{A}^{2}-I_{C}^{2}\right)^{1 / 2}-\left(m c^{2} / e\right)\left[I_{A} / I_{C}-1\right] \\
& \times\left\{\left[2\left(I_{A} / I_{C}+1\right)\right]^{1 / 2}-1\right\} .
\end{aligned}
$$

This approximate result, which can be applied no matter where the MITL is operating on Fig. 14, can be substituted for a voltage divider waveform measurement, which has been found impractical in MITLs above about $2 \mathrm{MV}$. Because both electrodes in an IVA-both within the adder cells and beyond-appear externally to be at ground potential, the currents, by contrast, are easily measured.

One or two other points are noteworthy in the design of negative magnetically insulated IVAs. One is that the discussion here has assumed that the radial feeds in each cell operate below electron emission thresholds. If electrons were to be generated in these feeds they would enter the bore, perturb the flow, alter the impedance, and probably flow to the load region. There they would arrive as electrons with energies corresponding to less than total voltage and would probably not be useful. The construction of these radial feeds is therefore designed to keep the electric fields at the cathode conductors less than electron emission thresholds, and this is discussed in Sec. III C 2. (a)
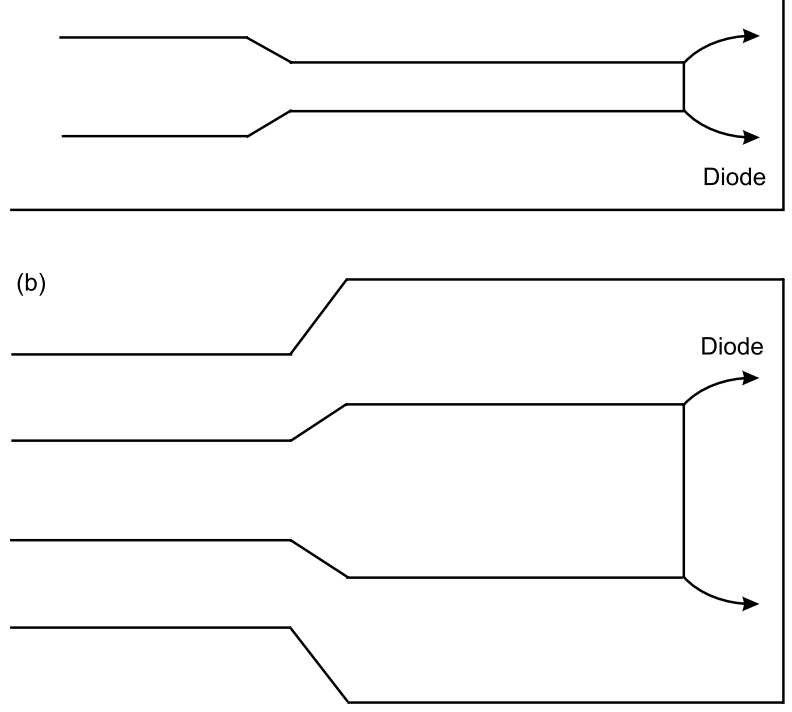

FIG. 19. MITL transitions (a) impedance increases; (b) impedance kept constant while diameter increases.

On the other hand, it is desirable that the magnetically insulated cathode stalk emit electrons readily, at as low an applied electric field as possible. Nonuniform emission from the stalk could lead to asymmetric flow, which might in turn lead to more sheath current or to actual loss of current to the outer of the bore. To promote ready electron emission, stalks are often coated with carbon, from which space-charge-limited electric emission turns on quickly and fully at fields of less than $200 \mathrm{kV} / \mathrm{cm}$.

The design of the magnetic insulation described assumes that the pulse power sources drive each cell with identical pulses and at exactly the prescribed timing, i.e., each cell is driven at the time when the pulse arrives at the cell from the upstream adder, all pulses arrive synchronously at the load, and that the drive is symmetric and azimuthally uniform around the bore to be consistent with magnetic insulation. The pulse power design usually attempts to minimize timing errors (to be discussed later); large undesirable effects of mistiming on magnetic insulation have not been encountered, and what levels are tolerable is only just beginning to be investigated. Achieving an azimuthally uniform feed of the cell bore is largely a matter of cell design and is discussed in Sec. III?C?3.

\section{The positive magnetically insulated IVA}

In a positive IVA, electrons are emitted not from the stalk but from the outer conductor of the bore, especially from the negative corner of each radial feed where the electric field is enhanced. As in a negative IVA, magnetic insulation can prevent such electrons from immediately crossing between the outer and the inner conductors and can cause them instead to propagate towards the load. But 
electrons emitted between radial feed gaps do not have the energy to return to the outer of the bore once they have passed the next radial feed. They must therefore eventually cross to the inner, arriving with an energy corresponding to less than the full voltage, and thus they are not useful. Thus the magnetically insulated positive voltage adder tends to be less efficient. Electron flow in a positive adder tends to fill the bore, in contrast to the negative adder where it stays close to the stalk. This is illustrated in Fig. 20(a), taken from Ref. [37]. In general, the operating impedance of the bore will be lower.

One consequence of electrons being emitted from the outer between cells and crossing to the inner is that the current loading the cells decreases along the adder in the direction towards the load, and therefore the cells nearer the load generate increased voltages.

If the positive adder is electrically short compared with the pulse, use of a higher than matched wave impedance

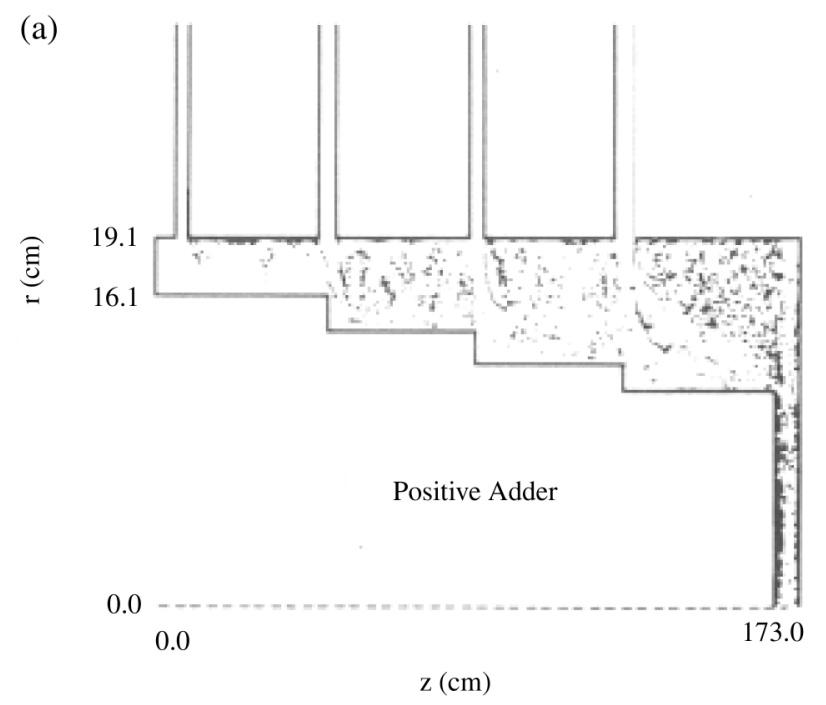

(b)

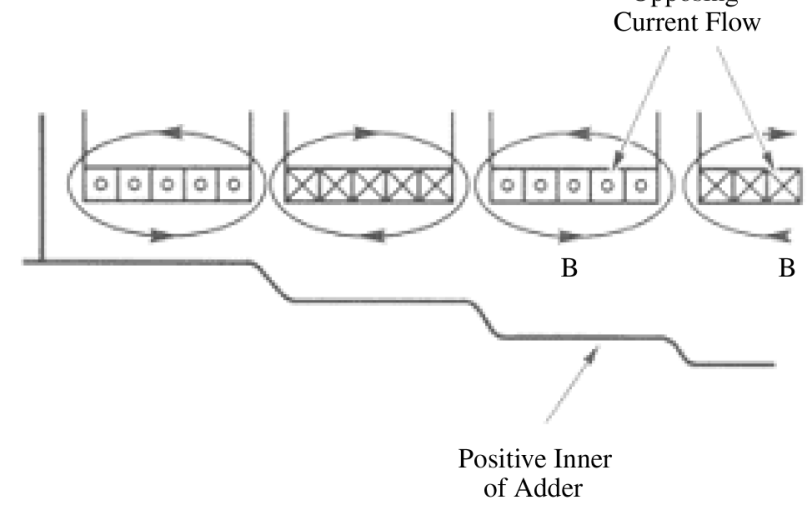

FIG. 20. (a) Distribution of sheath electrons for positive polarity four-stage adders from particle-in-cell code simulations. (b). Sketch of a positive polarity induction voltage adder using alternating-sign magnetic fields to trap sheath electrons within each adder section. for the stalk can reduce the unusable distributed electron flow to low levels, $\leqslant 5 \%$ in the four-stage Helia [38]. Even Hermes-III, where the adder is longer than the pulse, positive polarity tests [39] suggested that only $\sim 30 \%$ of the current was in the form of electrons emitted from between the radial gaps, and since the emission regions were distributed along the adder this represented only $\sim 15 \%$ of the energy. A relatively long positive magnetically insulated adder was successfully used to drive ion diodes in Sabre [40].

It is possible that electron flow from the outer into the bore could be suppressed by shaping the electrodes or by adding positive-potential electrodes just upstream or each radial feed gap [41], or by applying external magnetic fields, as suggested in Fig. 20(b). Alternatively, schemes other than magnetic insulation can be considered for positive adders.

\section{Other adder bore insulation schemes}

Vacuum insulation can be used in the adder bore without appealing to magnetic insulation, provided that electric fields on negative surfaces are kept below electron emission thresholds. This is the case in LIA injectors, where because the current is low and inductance is not a major concern, the spacings in vacuum can be made large to keep electric fields very low. Practically maintainable bare metal surfaces begin to emit significantly under tens of nanosecond pulses at fields above $150-200 \mathrm{kV} / \mathrm{cm}$. Anodized aluminum has (shown first by Maxwell Labs, unpublished) an emission threshold of over $300 \mathrm{kV} / \mathrm{cm}$, and this and other beneficial surface treatments are discussed in Ref. [42]. Anodized aluminum is widely used to suppress emission in the radial feeds of both negative and positive IVAs and can also be used to suppress emission from the outer conductor of the bore of a positive adder, as in the Cygnus adder [3] and Sec. IV B. (Fields on the positive stalk are not a concern.) It has not been used on the stalk of negative adders, partly because of the effectiveness of magnetic insulation in this case, and partly because fields on the stalk are higher. In a positive adder, the approach of suppressing emission may be chosen because magnetic insulation is less useful or less efficient, as discussed in Sec. III B 2. It also is more readily achievable because the cathode, being the outer, has lower electric fields and can more readily be designed not to emit, though field enhancement regions at the negative corners of the radial feeds must be considered.

Design fields of up to $300 \mathrm{kV} / \mathrm{cm}$ have been used with anodization. However, the anodization can be damaged, e.g., by late-time discharges, and regions that are hard to reanodize should probably be designed at more like $200 \mathrm{kV} / \mathrm{cm}$, as in Cygnus. For a matched adder, $F=$ $200 \mathrm{kV} / \mathrm{cm}$ on the cylindrical bore surface corresponds to a traveling wave current $F r / 60 \sim 3.3 r$ (kA). This current may be exceeded at the diode by designing the 
bore with an impedance higher than the load; this results in the bore being inductive, but there is little penalty in efficiency, especially if the adder is electrically short. Cygnus, with a $19 \mathrm{~cm}$ radius bore, delivers $\sim 65 \mathrm{kA}$ to a $2.25 \mathrm{MV}$ diode, and is designed to have almost $3 \mathrm{MV}$ present at the third cell due to inductive drop, which is increased by the fact that the diode impedance is falling.

Note that when the adder is designed below the emission threshold, azimuthal uniformity of drive is not important as it is when using magnetic insulation.

Fluid insulation can also be considered in the adder bore. This was considered in Ref. [24] and has been implemented using oil in RHEPP [43]. Liquids can operate at fields higher than $200 \mathrm{kV} / \mathrm{cm}$ for pulses of order tens of nanoseconds, and though fields on both the inner and the outer must be considered, an oil-insulated bore of radius $r(\mathrm{~cm})$ may be able to deliver currents approaching $10 r(\mathrm{kA})$, while use of water insulation, which has been considered for the Jupiter IVA [44] can allow currents of order $50 r(\mathrm{kA})$.

When fluid insulation is used in the bore, a full voltage vacuum insulator stack must be used at the bore output. The advantage of reduction of the total insulator inductance that is present in an IVA with a vacuum-insulated bore is lost (see Sec. III A), but the other advantages described in Sec. III A are preserved.

\section{Induction cell design}

In this section we describe the design of an induction cell, the remaining feature special to the IVA. The pulse lines and the remainder of the pulse power system outside the cell may be regarded as common to other types of pulse power systems, though their attributes that are of particular interest for their use in IVAs will be discussed in Sec. III D and illustrated in Sec. IV in describing actual system designs.

The example cell chosen here is that of the negative adder RITS [30]. This cell is designed to drive a vacuum insulated bore and to use magnetic insulation.

\section{General design and function}

The 1.35 MV RITS cell is shown in Fig. 21. The function of the cell is to accept the high voltage power pulse (1.35 MV for $70 \mathrm{~ns}$ in RITS) from the pulse forming line that drives it, through an aperture in the outer cylinder cell surface into the oil-filled outer region of the cell, and to deliver this pulse to an annular gap in the adder bore, the inner cylindrical cell surface, in vacuum. The high voltage pulse insulation makes the transition from oil to vacuum at the vacuum insulator stack seen at one end of the cell. The pulse then passes through a radial feed in vacuum and enters the evacuated bore of the adder, which is $39 \mathrm{~cm}$ in diameter in RITS. The twelve pulses from the twelve cells of RITS are designed to add to deliver $16 \mathrm{MV}$ to a cathode stalk that threads the adder bore. The stalk then delivers this voltage and $150 \mathrm{kA}$ to the diode for a $70 \mathrm{~ns}$ pulse.

As we saw in Sec. II A, a consequence of the configuration that places the gaps in the inner cell surfaces in series in the vacuum while maintaining the continuous outer surface of the succession of cells as a closed ground conductor is that the high voltage conductor entering the

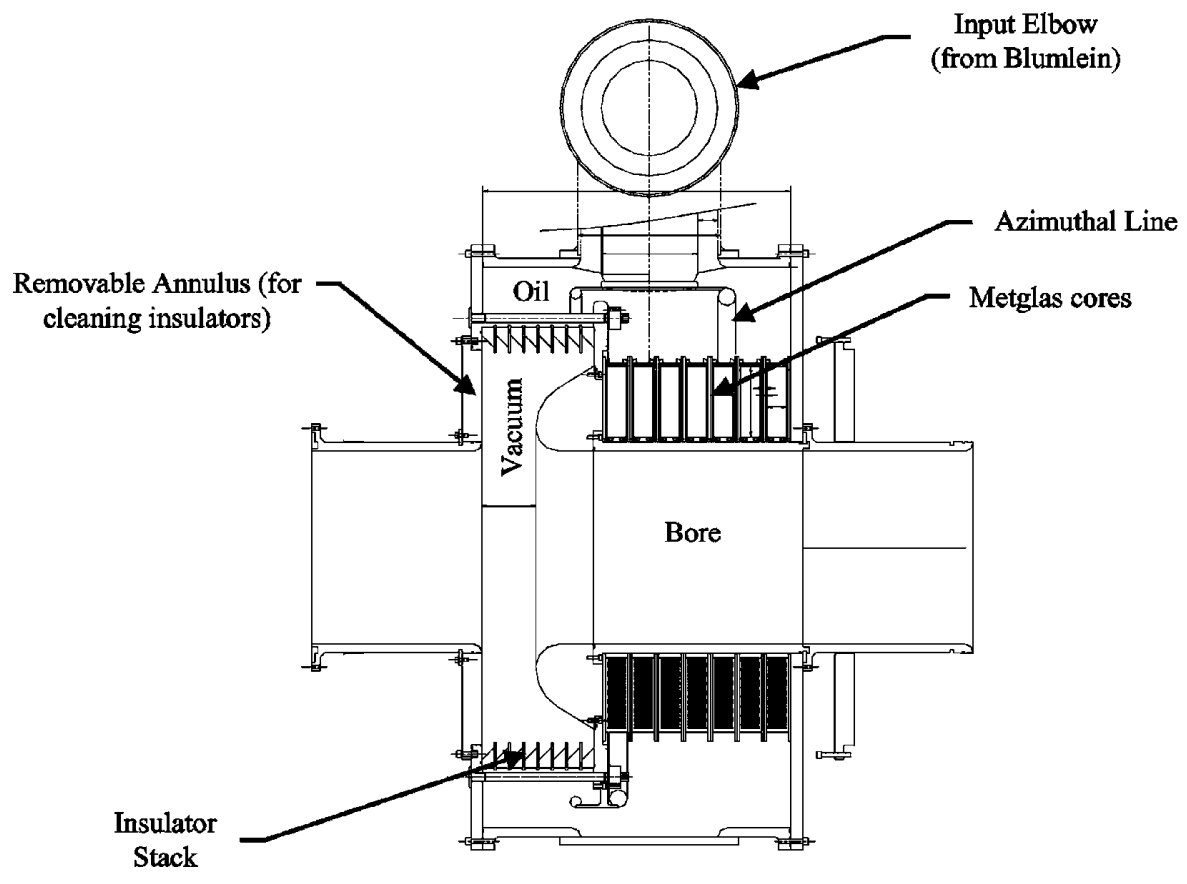

FIG. 21. RITS cell. 
vacuum bore must also connect back to the outer ground surface of the cell through the inner conductor of the cell and the farther end plate of the cell. In order to keep the loss current that flows from high voltage to ground through this connection path to a level low enough that it does not load the high voltage drive pulse down unacceptably, the inner conductor is surrounded by annular ferromagnetic cores, "Metglas," a product of the Hitachi Corporation (formerly of Honeywell Corp, formerly of Allied Corp) [45] in RITS. As long as the cores are not magnetically saturated, they present a high reactance against the loss current that threads them. The Metglas cores (seven in RITS) fill a cavity that occupies most of the cell length, and their insulation uses the same oil that is in the outer cell region. They are wound from many turns of Metglas ferromagnetic tape separated by Mylar insulation (other plastics and paper have also been used), and the small radial spaces between these layers are filled with oil in an initial vacuum impregnation of the whole cell.

The cell and its section of adder bore thus feature several different types of insulation, each with different design criteria. In the outer region of the cell, the insulation is pure oil. In the cores it is a combination of oil and a solid film, e.g., Mylar, and the insulation criteria used there are affected by the sharp edges of the Metglas tape. The vacuum insulator stack has its own criteria for avoiding flashover of the solid-vacuum surface. Between the insulator and the bore, in the radial feed, electric fields are limited by needing to avoid emission from the negative high voltage conductor. This is often (as in RITS) made from anodized aluminum to maximize the safe electric field on its surface. On the outer surface of the adder bore in a negative IVA like RITS, both of the conductors entering from the cell become positive with respect to the cathode stalk that threads the bore and are therefore capable of withstanding electric fields much greater than those that are present. The stalk itself is negative and has surface fields so high that electron emission cannot be prevented; magnetic insulation is therefore used in the bore, as discussed in Sec. III A.

A different insulation scheme has been used in induction cells of two LIAs, SLIA [46] and AIRIX [47]. There the vacuum insulators are placed at the input drive ports on the outer surface of the cell, and vacuum insulation fills the cell cavity as well as the bore. A SLIA $350 \mathrm{kV}$ cell has four such ports at $90^{\circ}$, and the $250 \mathrm{kV}$ AIRIX cell has two at $180^{\circ}$. It is probably practical to place the insulators at these locations only when the voltage is in this relatively low range, which is not typical of IVAs. Also, vacuum insulation throughout the cell is compatible with the ferrite cores used in SLIA and AIRIX, but not with the tape-wound oil-impregnated cores usually used in IVAs. An advantage of this "vacuum cell" for LIA use is that the ferrite is more directly exposed to the electron beam and tends to damp high frequency ( $\sim 1 \mathrm{GHz})$ modes in the cells that can result in beam transport instabilities.

There is considerable latitude in choosing the cell voltage in an IVA. Historically, $1 \mathrm{MV}$ was chosen for the proof-of-principle Helia and was maintained for Hermes-III, which partly motivated Helia. The choice of $1 \mathrm{MV}$ was influenced by the fact that the technology of water lines fast charged to 2-3 MV with self-closing output switches was well established at Sandia and gave $\geq 1 \mathrm{MV}$ output voltages. In the design of RITS, it was desired to increase the cell voltage in order to reduce part count. A voltage of $1.35 \mathrm{MV}$ was chosen because the charged pfl voltage then approached $3 \mathrm{MV}$, the edge of experience with pfls using self-closing switches, and because at the design current of $150 \mathrm{kA}$ a higher voltage would have raised the impedance beyond the range for which coaxial water pfls are suited (RITS pfls are $7.8 \Omega$ ). Other points that need to be considered if the cell voltage is raised further (e.g., for larger currents) are that the advantage of reduced total inductance in the vacuum (discussed in Sec. III A) is reduced, the cell cores (see Sec. III C 4) may become harder to build or to grade, and the pfl switch inductance and rise time may increase. Some of these considerations are less important for longer pulse durations, and $\sim 2 \mathrm{MV}, \sim 2 \mathrm{MA}$ were cell parameters adopted for Jupiter [44]. The designs of the various parts of an IVA cell are discussed further below.

\section{Vacuum insulator and radial vacuum feed}

In a magnetically insulated negative IVA, the insulator stack is placed at some radial distance out from the bore, partly in order to be able to shield it from electrons traveling radially outward from the bore when the initial electron loss front propagates down the adder. This shielding is completed by extending a conductor axially across the insulator stack immediately inside it, as seen in Fig. 21. In order to help grade the voltage uniformly along the insulator stack, this conductor extends from the high voltage side, because that is the side on which equipotentials approach the stack externally and where they would otherwise tend to be bunched. This conductor closes up the vacuum spacing inside the insulator and in this way also reduces inductance. Decreasing the cell inductance can be another reason to place the insulator stack at a large radius, towards the outside of the cell. Because the insulator stack must operate at relatively low electric fields and so must have a large high-voltage-to-ground spacing, it tends to dominate the cross-sectional area of the vacuum region seen in Fig. 21 and hence dominate the vacuum inductance.

Although the cell could be designed to accept either positive or negative pulses and deliver them with correct polarity to the bore (by orienting the cell so that the gap 
in the bore is at the appropriate end), most pulse power drivers are more easily designed to deliver negative pulses, so the conductor that shields the insulator stack, being the high voltage conductor, is negative. This means that the electric fields on its surface have to be kept below the electron emission threshold at its closest approach to ground - the flat or nearly flat region of the radial feedand on its field-enhanced corners.

The electron emission threshold of conductors can be increased to $200-300 \mathrm{kV} / \mathrm{cm}$ by using a very smooth or polished conductor surface. However, it is impractical to maintain a very good finish on the cathode plate of the radial feed because of the effects of late-time breakdowns that typically occur as the residual energy in the pulse power system rings after the main pulse, and in many cases because of debris from the diode region of the IVA depositing on the conductor. To remachine or (say) electropolish the surface would require the cell to be both removed and disassembled, and this is so difficult that it is not considered an option. One way to raise the emission threshold to $>300 \mathrm{kV} / \mathrm{cm}$ is to use an anodized aluminum surface, as discussed in Sec. III B 4. This is reasonably hard wearing, but the insulating surface layer formed by anodization can be punctured by discharges, and mechanisms to repair it in situ have not been established. Therefore while design fields as high as $250-300 \mathrm{kV} / \mathrm{cm}$ have been employed on the cathode plate, at least on its corners, by using anodized aluminum (on Helia, for example, Ref. [26]), a better design field is probably $200 \mathrm{kV} / \mathrm{cm}$.

On a negative adder such as RITS, the anodization on the cathode plate stops at a point just inside the corner where this electrode surface enters the cylindrical vacuum bore. There the field changes polarity because the conductor is positive with respect to the cathode stalk, so that much higher fields are safe. On a positive adder the outer of the bore is negative; anodization may continue, as it does on Cygnus [3], along the length of the adder bore. In this case the corner where the negative radial conductor enters the bore may need a large radius in order to avoid too much field enhancement.

The RITS cell insulator stack itself uses a standard multistage design with $\sim 45^{\circ}$ plastic insulators and intermediate metal grading rings. The grading ring tips in the vacuum are positive with respect to the nearby cathode electrode and hence need no special consideration to avoid electron emission. They may be made of anodized aluminum anyway, in order to reduce emission on voltage reversals after the pulse.

The flashover strengths of insulators with low permittivity (2-3) are all similar; acrylic and Rexolite (a proprietary formulation consisting mostly of cross-linked polystyrene) have been widely used. Of the two, Rexolite has better vacuum properties (though this is mostly because it absorbs less water, which is not present in most IVA cells), and it is very resistant to electric bombardment, so it is probably the best material. It may also have a slightly higher flashover strength.

Design criteria for the electric field $F(\mathrm{kV} / \mathrm{cm})$ on the insulator stack, which determines its length, usually start from Martin's well-known flashover equation [48]

$$
F t^{1 / 6} A^{1 / 10}=175,
$$

where $t$ is the pulse duration in microseconds at the $89 \%$ level and $A$ is the area in $\mathrm{cm}^{2}$, though other equations have also been proposed [49]. This equation is conventionally used to infer that the probability $p$ of failure on a particular pulse is given (if $p \ll 1$ ) by

$$
p \sim 0.5\left(F t^{1 / 6} A^{1 / 10} / 175\right)^{10} .
$$

However, the computation of probabilities is actually more complex [50]. The IVA is a good example of a system in which it is not necessary to use the area of all insulators in the system to estimate the probability of breakdown. This is because flashover of one stage in one cell does not affect the total remaining area, which is mostly in other cells. Instead, because of this consideration and because of the redundancy that results from the multistage design of the stack, i.e., its ability to operate with one or more insulators flashed over, the author has suggested in Ref. [50] that if Martin's equation is taken to be true for individual insulators (as Martin intended), this implies that for a stack of at least six insulator stages it is conservative to take the probability of complete failure of the stack as

$$
P \sim 2 p_{1}^{2},
$$

where $p_{1}$ is the probability calculated for a single flashover in one stack using the Martin-derived probability equation immediately above. This allows higher design values of $p_{1}$ and hence higher stresses.

A significant probability of stack flashover, or of other types of breakdown in a cell, may be tolerated recognizing that in an IVA even the complete breakdown of one cell reduces the load voltage by only a certain amount. In the twelve-cell RITS, it is estimated from circuit simulations that a stack flashover decreases the (matched) load voltage by 1 part in 24 , because the open-circuit driver voltage falls by $1 / 12$ but so does the driver impedance. However, it is possible that asymmetric current flow through the flashover could perturb power flow in the bore.

For the vacuum insulator stack to insulate reliably as estimated above, it must be clean. In many IVAs, the diode region creates debris on each pulse in the form of vaporized or fragmented anode and cathode material. Even though the diode may be far from any insulator stack, high velocity debris particles can reach all parts of the adder. Many will fall into the insulator stacks before coming to rest. 
In an application where such diode debris is frequently produced, provision can be made to clean the insulator relatively easily by removing an annular region of the anode side of the radial feed. This design feature, seen in Fig. 21, was first included in Cygnus and is further described in Ref. [3].

\section{Current Symmetrization and the azimuthal oil line}

If magnetic insulation is used in the evacuated bore of the adder, this requires that a relatively uniform magnetic field surround the central cathode stalk. Where the high voltage conductor and end plate of the cell enter the bore at the vacuum gap, the magnetic fields near the thick conductors are proportional to the surface current density, and this current density must therefore be made relatively uniform around the gap in the inner surface of the cell.

In the RITS cell this uniformity is effected, in spite of the fact that all the current enters at the single location of the feed from the pulse forming line, by the design of the azimuthal transmission line conductor, which is indicated in Fig. 21. This conductor is driven with high voltage at the single location where the pulse enters the cell, and it forms with the outer cell surface an azimuthal transmission line that runs all round the cell circumference. The high voltage azimuthal line conductor is connected to the high voltage electrode of the vacuum insulator stack only at specific regions of the circumference. On RITS a slot (indicated in Fig. 21) separates these two conductors on the side of the cell where the current enters, forcing the current to flow about $90^{\circ}$ around the cell before it can flow through the insulator stack. The azimuthal line and high voltage stack are connected over the remainder of the circumference, opposite to the input point. A more complex slot geometry may be needed, e.g., multiple slots, e.g., in cases where the current has less natural tendency to symmetrize in the radial vacuum feed because this is smaller in relation to the vacuum bore.

The high voltage conductor of the azimuthal oil line has a maximum width on the side of the cell where the pulse is injected into the cell. In this region its characteristic impedance is designed to be such that the two current paths extending away from the feed roughly match the impedance of the drive pfl. As the high voltage conductor continues around the opposite side of the cell its width decreases, increasing the wave impedance as the current being carried by the azimuthal line is reduced by the current flowing through the vacuum insulator. The profile of the width of the RITS azimuthal line was optimized to give good current uniformity in the bore and to minimize the increase of rise time produced by the cell.

MRC's three-dimensional PIC-code LSP [32] (without electrons) was used to design and study the azimuthal oil line in the cells in RITS and in an AWE IVA [34] cell design. Figure 22 shows the LSP model of the AWE cell

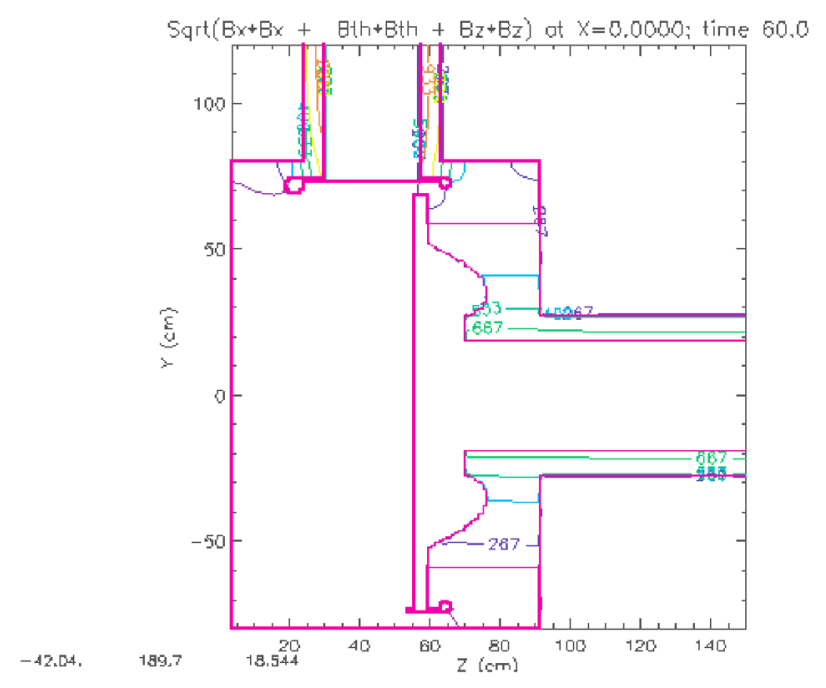

FIG. 22. (Color) LSP model of cell.

in a section through the feed. In the model, the cell drives a single $\sim 22 \Omega$ axial vacuum transmission line and most closely represents the first cell of the adder. The first cell is chosen as the basis of the azimuthal line design because calculations for RITS showed that a given azimuthal line design will produce greater nonuniformities in lower impedance coaxes. The worst case is thus the first cell. This can be understood by considering that in other cells the greater inductance of the higher impedance bore that extends from the cell in both directions tends to make the current become more uniform as it flows through the cell's radial vacuum feed before it reaches the bore.

The cell model of Fig. 22 was driven by a $14.4 \Omega$ voltage source with a sinusoidal rise, $19.5 \mathrm{~ns} 10 \%-90 \%$, somewhat faster than the actual rise time anticipated. Figure 23 shows as a function of time axially the current density $7 \mathrm{~cm}$ into the bore, at $0^{\circ}, 10^{\circ}$, and $180^{\circ}$ from the

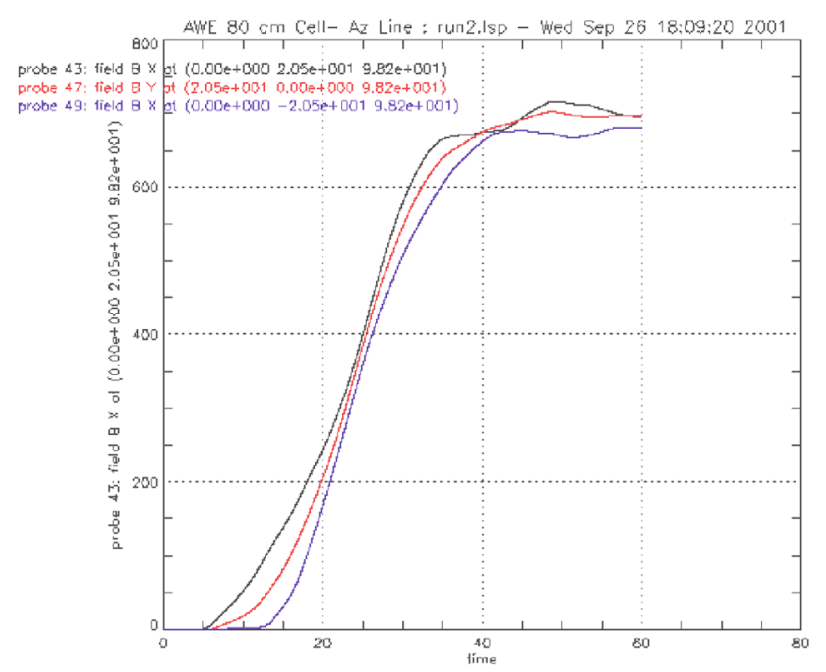

FIG. 23. (Color) $B_{\theta}$ in MITL measured $7 \mathrm{~cm}$ beyond anode plate. 
feed. It is seen that except transiently on the rise the current density is quite uniform, with a $\sim 2.5 \%$ total spread.

This approach to the design and the modeling of the cell for uniform current density is being validated in the RITS test program at Sandia. It is hoped to find experimentally how much nonuniformity in the current can be tolerated before electron losses occur or before electron flow in the bore is significantly increased at the expense of bound current in the stalk. The azimuthal line can be modified to make the current less uniform or can be removed entirely. The results for a single cell [51] show reasonable agreement between LSP and experiment. They also show that with azimuthal current uniformity significantly worse than the best design available using LSP, there were no significant magnetic insulation losses. Losses of order $10 \%$ were recorded when uniformity was made still worse by removing the azimuthal line. This work is now being continued with three cells.

RITS, which uses the approach described of a single tapered azimuthal oil line inside the cell to assure uniform current feed to the bore, has only one pulse forming line feeding each cell. Previous IVAs had two or four pulse lines per cell and used systems of discrete constant impedance transmission lines internal to the cell to both make the current feed to the bore uniform and to average out any time differences between the feeds. This approach was used in the first magnetically insulated IVA, the 4 MV "proof of principle" Helia [26]; the azimuthal line network, shown schematically in Fig. 24, took the input from two lines and delivered it as four averaged pulses to four locations $90^{\circ}$ apart on the insulator stack. From these points on, the current was assumed to spread out enough as it flowed radially inwards to become adequately uniform by the time it reached the bore. A similar but more complex discrete transmission line network was used to mix the four input pulses on Hermes-III [52]. No electron losses were observed on Helia and Hermes-III.

A single-point cell feed was used on RITS to facilitate changing between the various planned accelerator configurations [28]. Now that it has been devised, a singlepoint cell feed has been found to be convenient for the IVA architecture design in general. However, it is practical only for IVA currents up to the region of the $150 \mathrm{kA}$ of RITS, because for higher currents the width of an azimuthal line that matches the pfl driver is too great to fit in the cell length, at least for $\sim 1.5 \mathrm{MV}$ cells. But a single water pfl may not conveniently deliver much more than $150 \mathrm{kA}$ in any case. For higher current IVAs more than one cell feed may be needed and more than one azimuthal line. An azimuthal line like that of RITS but with more feed points can then be used. However, a configuration like that for Helia and Hermes-III may be preferred. An approach like that for Helia and Hermes-III may result in a shorter cell rise time; cell inductance may be lower because the insulator stack carries current more uniformly over its circumference, and rise time smearing due to azimuthal transit times is reduced.

\section{Ferromagnetic core region}

The largest region of the induction cell, the cavity, contains ferromagnetic material whose purpose is to reduce the current flowing from the high voltage electrode at the vacuum insulator stack to ground via the outside of the metal cylinder that forms the vacuum bore. This current is termed the loss current. The size of the cell is in most designs dominated by the need to include a large enough cross sectional area $(A)$ (in the view of Fig. 21) that the ferromagnetic material does not saturate during

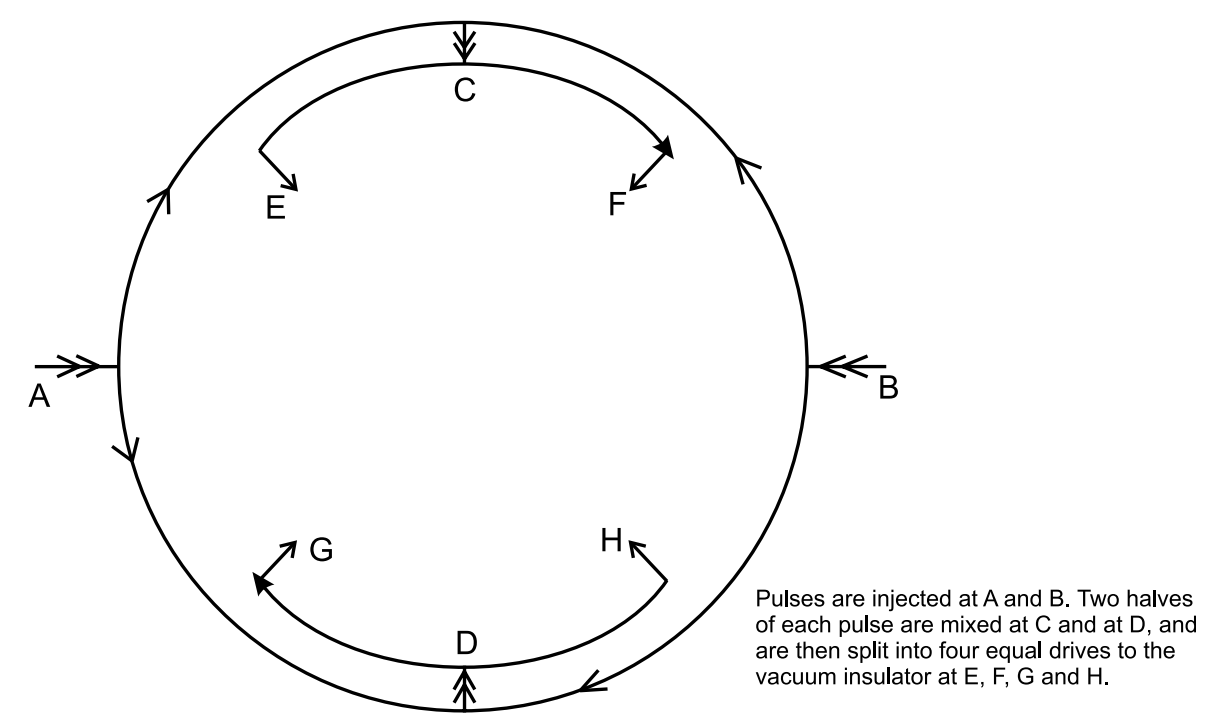

FIG. 24. Schematic of system of discrete transmission lines used to mix and symmetrize two pulse inputs in the cell of Helia. 
the high voltage pulse of voltage $V$ and duration $T$. The condition for no saturation is that

$$
A(\Delta B)>\int_{o}^{T} V d t
$$

where $\Delta B$ is the flux density swing that the core material can undergo without saturation. The relevant $\Delta B$ is usually the change from remanence in the direction opposite that of the pulse to a value where the magnetic field generated under the pulse has increased to a value that corresponds to a loss current approaching excessive loading on the generator. This is illustrated in the schematic $B H$ loop shown in Fig. 25, which shows that $\Delta B=$ $B_{\text {rem }}+B_{\text {sat }}$, the sum of the remanent and effectively saturated $B$ values. The core is usually "reset" to $B_{\text {rem }}$ with a dc (seconds duration) current some time before the pulse; the part of the $B H$ trajectory shown as a full line in Fig. 24 is then traversed under the cell voltage pulse. Application of a constant voltage would correspond to moving up the $B$ axis at a constant rate (constant $d B / d t$ ) so that (as illustrated in Fig. 24) the vertical axis can be roughly regarded as a time baseline with the current waveform represented by the shape of the horizontal displacement of $H$ from zero. The average $H$ value present during the pulse determines the average loss current and hence the lost energy.

The $B-H$ loop that is important here is not the dc $B-H$ loop but the one that applies for the short duration pulse of the IVA. This has the same $\Delta B$ as the dc $B-H$ loop, but $H$ values are larger and increase as the pulse duration is shortened. Approximate models of dynamic or timedependent $B-H$ curves that can be incorporated into circuit simulation codes have been developed by Schlitt (Leland Schlitt Consulting Services) and have been used (principally at Titan Pulse Sciences) to model both IVA cavities and saturable cores used as switches.

The principal choice of core material is between an iron tape and a ferrite. The cores in the RITS cell, shown in Fig. 21 are of Metglas 2605CO, an amorphous iron tape made by the Hitachi Corporation. IVAs tend to use iron tape because it has a much larger $\Delta B$ than ferrite, allowing the cell to be much smaller. Considering only the materials themselves, Metglas 2605CO has $\Delta B=B_{\text {rem }}+$ $B_{\text {sat }} \gtrsim 3 \mathrm{~T}$, compared with $0.5-0.6 \mathrm{~T}$ for ferrites. The effective $\Delta B$ in the cavity as a whole is reduced in the case of Metglas because (1) the Mylar insulation between turns typically results in a radial packing factor of $\sim 0.6$; (2) insulation spacing needed between cores results in an axial packing factor typically $\sim 0.85$; and (3) there must be bulk oil insulation between the outer turns of the cores and the surrounding conductors, which in Fig. 21 are the azimuthal transmission line and the cell ground. These three factors typically reduce the overall $\Delta B$ by a factor of about 2 , from $3 \mathrm{~T}$ to around $1.5 \mathrm{~T}$. When ferrite is used, it is in the form of solid annuli, or "pucks," that are typically $2.5 \mathrm{~cm}$ thick. Because the pucks are insulators they need only about $1 \mathrm{~mm}$ spacing between them to allow oil to enter, and they can extend over the entire radial distance from the bore to an outer conductor. Therefore for ferrite the average $\Delta B$ over the cavity can be more than $90 \%$ of the material $\Delta B$, i.e., $\sim 0.5 \mathrm{~T}$.

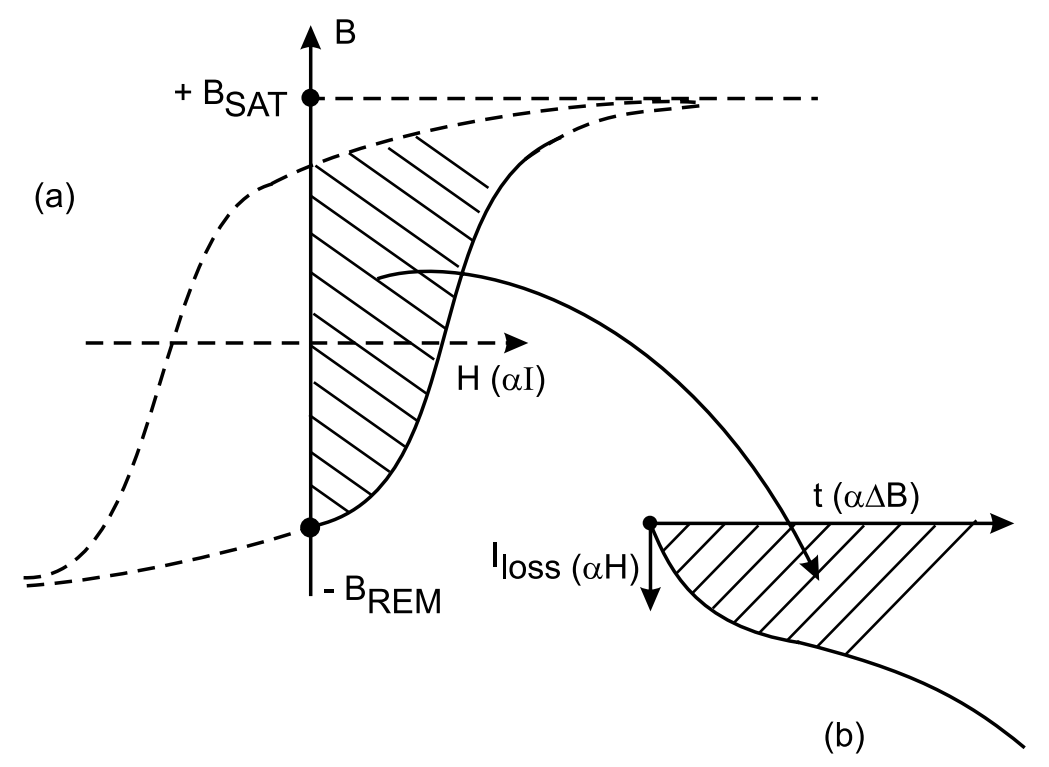

Note that width of B-H loop and amplitude of loss current increase as core is driven in shorter times.

FIG. 25. (a) Schematic $B-H$ loop, showing (full line) trajectory swept out during main pulse; (b) under constant voltage $(d B / d t)$, loss current waveform is a rotation of $B-H$ trajectory. 
Ferrites tend to be used in LIAs, where the load currents are often limited to a few to $10 \mathrm{kA}$ by beam transport instabilities. For $\sim 1 \mathrm{~m}$ diameter cores, the ferrite currents can typically be of the order of $1 \mathrm{kA}$, whereas a Metglas core operated at a large flux swing will typically draw $\sim 5 \mathrm{kA}$. This would make a Metglas-core LIA relatively inefficient. Also, a larger core current change as the $B$ - $H$ loop in Fig. 25 is swept out during the pulse and will make it difficult to achieve a flat acceleration voltage pulse, which is needed in a LIA for good beam transport. In spite of these considerations, in the second axis of DARHT [53], Metglas is used in a few kA LIA, simply because space is not available for enough ferrite to avoid saturation during the $2 \mu \mathrm{s}$ pulse. The relatively long pulse of DARHT-2 reduces the width of the $B H$ loop and reduces loss current to a considerable degree. Typical short pulse IVAs have currents of more than $50 \mathrm{kA}$, making the Metglas loss currents quite acceptable, and they have no voltage-sensitive beam transport considerations.

The best Metglas and ferrite cores tend to have fairly similar ratios of $\Delta B / \Delta H$, where $\Delta H$ is an average over a pulse of $\lesssim 100 \mathrm{~ns}$ duration. This ratio corresponds to the order of 100 in terms of gauss per oersted, but should not be referred to as a permeability. The cores behave rather like resistors under short pulses, absorbing energy and not returning much to the drive circuit as the current falls to zero, as an inductor would. Under a flat voltage pulse the core current may in fact have a substantial fast rising, flattopped component that is similar to what would result if the core were a resistor.

Because the materials have similar $\Delta B / \Delta H$ ratios, a Metglas core operated at a $\Delta B$ like that available in ferrite may have similar average $H$ and hence similar losses to ferrite. However, of the two core materials, ferrite would be preferred at low $\Delta B$, because it is cheaper. It is also easier to handle; Metglas cores may "telescope" and require care to keep the insulation impregnated with oil.

The advantage of an amorphous metal like Metglas over other iron tape materials is the high resistivity that results from the amorphous structure, which is created in a high speed solidification process in contact with a cold metal roller. The resistivity is about 4 times that of the metal in its normal form. Under the short $(<100 \mathrm{~ns})$ pulses the magnetic field propagates into the tape from its surface. The loss current is determined by the surface $E$ field and resistivity; it is higher for higher $E$ fields (which result in shorter saturation times) and lower with higher resistivity. The use of more, thinner laminations presents more total surface path length to the applied $E$ field and also decreases the loss current. Metglas is commonly used in $25 \mu \mathrm{m}$ thicknesses and is available as thin as about $15 \mu \mathrm{m}$. Permalloy $10 \mu \mathrm{m}$ thick has been used in Soviet LIAs; although this material is not amorphous, its losses are similar to those of $25 \mu \mathrm{m}$ Metglas because it is thinner. A number of types of Metglas are available from its maker, Hitachi. The highest flux swing is obtainable from $2605 \mathrm{CO}\left(B_{r}+B_{\text {rem }} \gtrsim 3 \mathrm{~T}\right)$, which has been used in many IVAs in addition to RITS. Vacuumschmelze's Vitrovac $7600 \mathrm{Z}$ has a flux swing $>$ $3 \mathrm{~T}$ and losses and $B H$ curve similar to $2605 \mathrm{CO}$. If lower loss is important, because of either a desire for high efficiency or difficulty in cooling, a lower flux swing material such as Metglas 2605SC $(\Delta B \sim 2.5 \mathrm{~T})$ may be preferred.

Metglas is typically used in $5 \mathrm{~cm}$ widths. When $2.5 \times$ $10^{-3} \mathrm{~cm}$ thick material of this width is driven through a $\Delta B$ of $\geq 3 \mathrm{~T}$ in $\sim 50 \mathrm{~ns}$ a laminar core develops a voltage of $\sim 100 \mathrm{~V}$ per turn. Insulating coatings are not readily available that can withstand such voltages, and insulating film is therefore wound up with the Metglas with the film typically $1-3 \mathrm{~mm}$ wider than the Metglas. This film, e.g., 6-12 $\mu \mathrm{m}$ Mylar (other plastics or paper have been used), can withstand much more than $100 \mathrm{~V}$, but nevertheless two layers of Mylar may be used in order to avoid pinholes in a single sheet shorting two layers of Metglas. A typical core may have many thousands of turns, and many breakdowns between layers can actually be tolerated. In effect, such a breakdown decreases the surface path for $E$ fields around the turns that are shorted together and only increases losses locally.

The designer of the Metglas core region must consider electric fields external to the Metglas cores, chiefly axial fields. The axial electric fields within the cores are negligible (as are azimuthal fields) because the tape is a good conductor. The design objective is to make the axial fields outside the cores fairly uniform and adequately low. The situation is illustrated for three cores in Fig. 26. In an ideal case where the end plates of the core region extend straight out to infinity, uniform fields between cores can be ensured by using equal spacings $d$ between cores and spacings $d / 2$ from the end cores to the end plates, Fig. 26(a). There are then equal and opposite "voltage drops" along the two sides of each core and no net charge on any turn. (By voltage drop, we mean what would be measured by a fast electrostatic voltmeter located in the region outside the cores connected across the core face.) Capacitance from the outer turns of the cores to end plates that wrap around the outside of the cores, Fig. 26(b), can induce charge on the outside of the outermost turn of the core, and this leads to unequal voltage drops on the two core faces and therefore different voltages between different pairs of adjacent cores. Wrapping the conductors round from both ends in a roughly symmetrical fashion and not approaching the cores too closely, Fig. 26(c), can avoid large disturbances to the desirable uniform grading of the cores. Use of unequal spacings between cores could then reduce residual field inequalities, but this has probably never been attempted.

Figure 27 shows an electrostatic field plot used to study core grading. The electromotive forces (emfs) applied to 


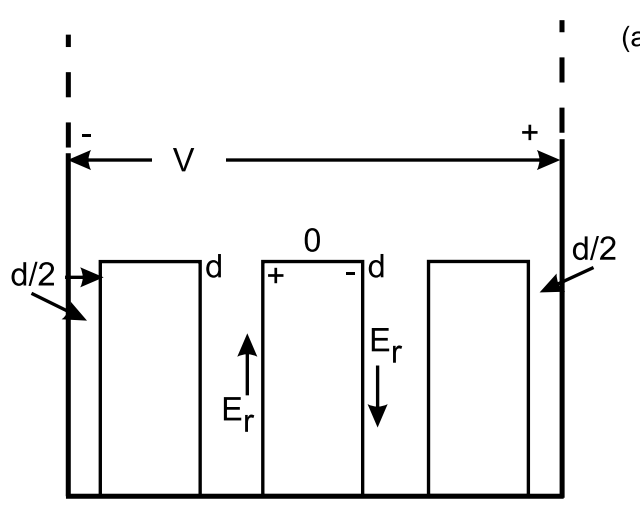

(a) Ideal Grading

Fields are equal and opposite on the two faces of a core, and so net charge on both inside and outside of the outer turn is zero. Axial fields between cores are equal, and are $\mathrm{V} / 3 \mathrm{~d}$. "Voltage drop" along each core face is $V / 6$.

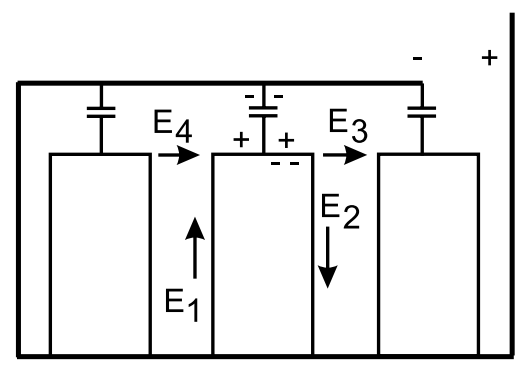

(b) Nonuniform Grading

Capacitances to negative conductor near outer turns of cores induces positive charge on the outside of the outer turn, and the negative charge resulting on the inside changes radial fields, making $E_{2}>E_{1}$ and $E_{3}>E_{4}$

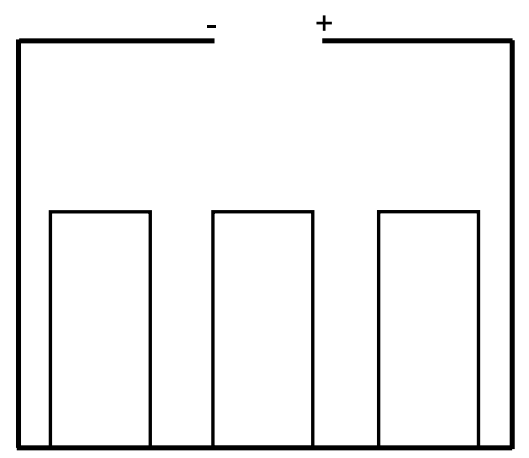

(c) Nearuniform Grading

Symmetry of outer conductors and their larger spacing to cores reduces uniformity only slightly.

FIG. 26. Effects of nearby conductors on field distribution in a set of three ferromagnetic tape cores.

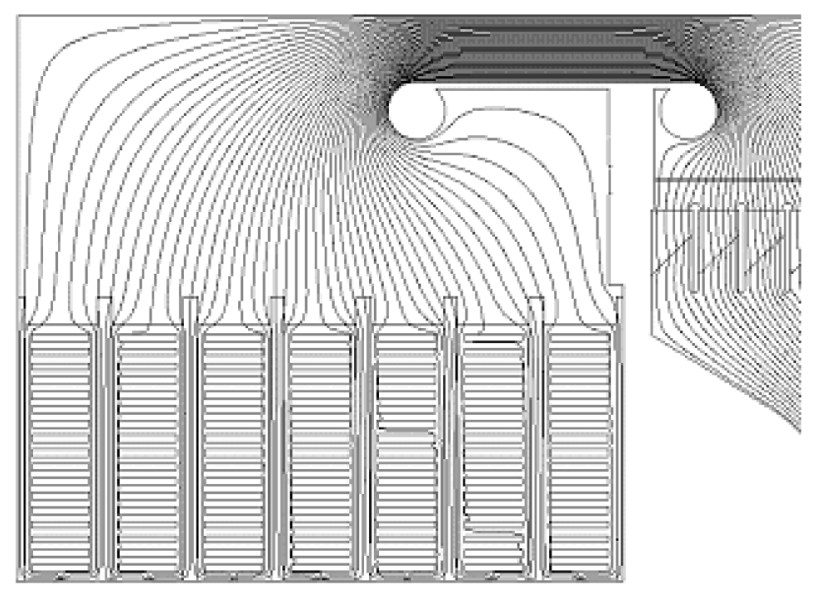

FIG. 27. Quasielectrostatic calculation of external potential distribution in core region. the cores are represented as a series of equal electrostatic voltage drops on the inner surface of each core-an artificial way of representing inductive emfs in an electrostatic calculation, devised by Schlitt in the Helia program [26]. The radial capacitance of the cores is represented by dividing each into a number of radial sections representing metal and dielectric with correct proportion and permittivity. Such a calculation correctly calculates the axial fields between outer regions of adjacent cores, where the axial fields are greatest; it does not correctly estimate axial fields at smaller radius or radial fields. By adjusting the geometry, a roughly uniform axial field is obtained at the outer edges of the cores. Typical design values for $\sim 50 \mathrm{~ns}$ pulses are $150-250 \mathrm{kV} / \mathrm{cm}$. Core separations are maintained mostly by plastic spacers, plus the Mylar that sticks out a few $\mathrm{mm}$ from between turns of Metglas. The fields are enhanced at the 
edges of the outer turns, and local shielding may be provided by adding conductors there with a few $\mathrm{mm}$ radius.

The assumption of uniform emfs around each core in Fig. 27 implies that the currents linking all cores are equal. This in turn implies that the charging of stray capacitances is complete; such charging may entail currents that link some cores only, so that these cores will have a larger emfs at first. This is a transient situation that will usually soon disappear, as core currents of a few kA will charge typical stray capacitances of $10 \mathrm{pF}$ or less to fractions of $1 \mathrm{MV}$ in a few ns.

Fields in board of the core outer regions require timedependent models to predict even when stray capacitances have been charged and the voltage is a constant flattop value. In such a quasisteady state, $H$ will fall off like $1 / r$, and the radial dependence of $E(\alpha d B / d t)$ will depend on the relation between $d B / d t$ and $H$. For example, for some core materials, $H$ increases more weakly than linearly with $d B / d t$, and this will lead to $E$ falling off faster than $l / r$. (Recall that inductive $E$ fields are of opposite sign at the two ends of a core, so this does not violate Gauss's theorem.) In any case, $E$ and $d B / d t$ will be larger, and hence saturation will be approached more quickly at the inside radius of the core. Saturation may occur at the core inner radius well before the end of the pulse and propagate outward. But if $A(\Delta B)$ for the core exceeds the volt seconds of the pulse, an outside region of the core will remain unsaturated at the end of the pulse.

Given a known time-dependent relation between $H$ and $d B / d t$, the radial $E$-field distribution and the propagation of saturation can be modeled by breaking each core into elements coupled by capacitances and applying the dynamic $B H$ model of Schlitt mentioned earlier. This has been done by Leland Schlitt Consulting Services under contract to Pulse Sciences, initially in the SLIA program. In such models of magnetic switches, which eventually saturate throughout, predictions of the distribution of energy deposition were in general agreement with measurements of temperature rise profiles. It was found that energy density can be greater at the outside of a core than at the inside because of the speed of final saturation there, and it may be that this is also true of peak radial electric fields in IVA cores even when they do not quite saturate throughout. The effects of axial and external capacitances can be included in such a model if desired.

The design of IVA core regions, as opposed to magnetic switches, usually does not need this detailed modeling using dynamic $B H$ loops. Though radial electric fields may be nonuniform and time varying, the use of $\sim 10 \mu \mathrm{m}$ per turn insulation allows the actual radial fields generated when $5 \mathrm{~cm}$ wide cores saturate in $\sim 50 \mathrm{~ns}$ to be withstood. Axial fields estimated by calculations like those in Fig. 27 as $150-250 \mathrm{kV} / \mathrm{cm}$ result in reliable operation. It may not be advisable to let the cores routinely saturate while cell voltage is still at or near peak, because large radial fields could result near the outside of the cores. While it is true that well-impregnated cores operate safely at similar fields in magnetic switches, the presence of air or solid insulation or field enhancement could result in discharges.

It is normal to vacuum impregnate an IVA cell with oil initially in order to ensure that air is eliminated and that oil penetrates between and inside the cores. An impregnation with oil after pumping for some time to the 1 Torr range or below will avoid air being trapped between the Mylar layers at the edge of each core. It is desirable to degas the oil separately before impregnation, because otherwise it foams up and liberates air when introduced into the evacuated cell. Cells need to be drained occasionally during IVA maintenance, and it is probably a good idea to vacuum impregnate again each time afterwards. Some IVAs have, however, been operated after filling without evacuation, without observing breakdown. Some air is likely in any case to remain trapped throughout a second or subsequent vacuum impregnation, so the case for always impregnating when refilling cells is not strong.

It is desirable that all Metglas cores within a cell be of the same material. Measurements of combinations of cores of different types of Metglas show that, as would be expected, their different relations between $H$ and $d B / d t$ can lead to very unequal distributions of emfs and to complete saturation of some cores well ahead of others, and higher fields will be generated between cores.

A number of techniques are used in resetting the ferromagnetic cores for each pulse. In IVAs, it is most common to connect a dc current source to the inner conductor of each cell in turn through a mechanical plunger, and inject a current (typically $\sim 100 \mathrm{~A}$ ) to produce a magnetic field of a few tenths of an oersted throughout the Metglas core for a few seconds. Where cells are driven from a common pulser, resetting them while they are connected in parallel may be difficult, not only because a larger total current is required but because dc contact resistances can make the reset current divide unequally between cores. In Cygnus [3], cells connected in parallel and driven by a single pfl are reset individually in turn; a plunger carrying the reset current disconnects each cell in turn from the common drive point or manifold as that core is being reset, ensuring that the cell receives all the current. An alternative would be to put small resistances in series with each cell to swamp the differences in core feed resistances, increase the reset current by the number of cells, and reset all cells in parallel.

When a Blumlein is used to drive IVA cells, the current charging the inner line of the Blumlein can flow around the core and reset it, Fig. 28. This can allow an "active" reset to reverse saturation rather than to remanence, increasing the available flux density swing from $B_{\text {rem }}+B_{\text {sat }}$ to $2 B_{\text {sat }}$. For this to occur it is necessary to switch the 


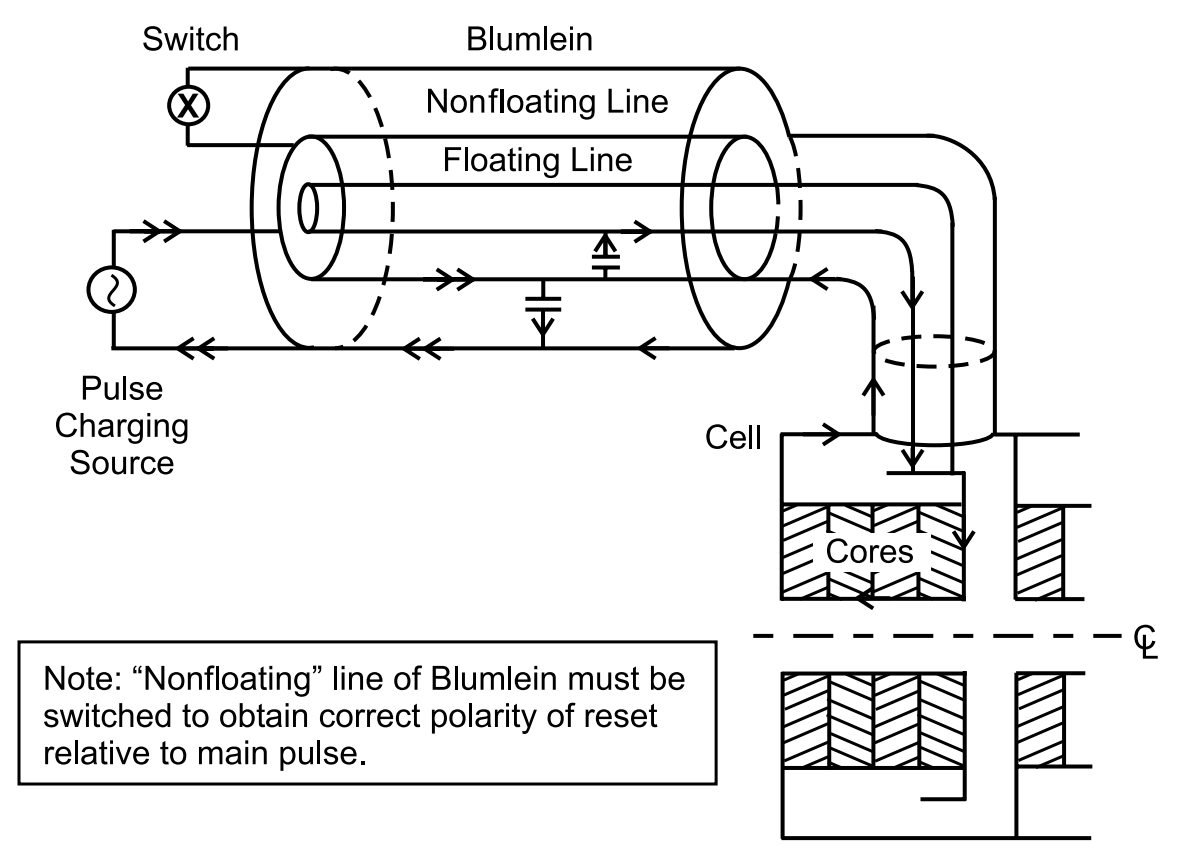

FIG. 28. Current charging the capacitance of the "floating" line of Blumlein (shown by arrows) can be used to reset cell cores.

outer line of the Blumlein in order to achieve the correct polarity of the prepulse current with respect to the main pulse. Also, the circuit must be designed to accomplish reset and then keep the core at or near saturation up to the time that the Blumlein switches out to generate the pulse. Along with active reset, it may still be necessary to use a $\mathrm{dc}$ reset to ensure that all cores are in the same remanent condition at the start of the Blumlein charge. This has been found unnecessary in the Blumlein driven, ferritecored FXR, [54], but it was found to be necessary in prototype circuits of Metglas-cored IVAs at AWE [55].

Even if a Blumlein is not used, active reset can be accomplished by dc or a pulsed current through an inductor permanently connected to the cell and large enough in value not to load the pulse, and this is done in DARHT-2 [52].

In a repetitive system, the use of a reset plunger is impractical, and a permanently connected inductor must be used, carrying dc or pulsed current or both.

\section{Some comments on pulse power for IVAs}

To go into details about the pulse power used to drive IVAs would extend this article well beyond its present length. Some examples of pulse power systems will be seen in outline in Sec. IV. Here we discuss particular features of the pulse power that may be important for IVAs. We address, in particular, two considerations: mistiming jitter between cell drivers, and prepulse.

\section{Driver design and jitter}

Historically, the first IVAs were driven by fast-charged simple water-dielectric pulse forming lines with self- closing water output switches. This included Helia [26] and Hermes-III ([52] and Sec. IVA). Measurements on prototype [56] and operational Hermes-III pfls showed that the individual pulse line switches had rms jitters of $\sigma=3-4 \mathrm{~ns}$. Since the diode voltage can be considered as the linear superposition of the pulse line outputs ( $80 \mathrm{pfls}$ were used in Hermes-III), this jitter can be translated as a $10 \%-90 \%$ rise time component $\sim 2.6 \sigma \sim 10 \mathrm{~ns}$, where the factor 2.6 corresponds to the $10 \%$ and $90 \%$ points of a timing-probability distribution that is assumed to be normal. On Hermes-III, the pulse power and the cell were both designed to have $10 \%-90 \%$ rise time of about $10 \mathrm{~ns}$ also, so the total diode rise time would be expected by a quadrature addition to average $\sim 10 \sqrt{3} \mathrm{~ns}$ or $15-20 \mathrm{~ns}$.

The Hermes-III pfls are fast charged (in $\sim 200 \mathrm{~ns}$ ) by intermediate store water capacitors, each synchronously discharged into a group of four pfls by a laser-triggered gas switch whose jitter, of the order of $1 \mathrm{~ns}$, is almost small enough to be neglected. These four pfls drive successive cells in the adder, and the cell spacing is such that ideally each cell should be pulsed about $2 \mathrm{~ns}$ after the one upstream in order for voltages to add without reflection and pulses to be simultaneous at the diode. If all four pfls charged by one intermediate store have the same average switch-out time, the four cell pulses will have average errors from an ideal timing of $+3,+1,-1$, and $-3 \mathrm{~ns}$ moving from downstream to upstream, where positive and negative signify pulses being late and early, respectively. This is equivalent to an rms error of $\sim 2.2 \mathrm{~ns}$ and so will contribute a little to the diode rise time also, but the quadrature addition suggests a very small increase, $\sim 0.1 \mathrm{~ns}$. This rise time contribution could in fact be eliminated by adjusting the individual pfl water 
switch spacings to make the average closure times ideal; because the contribution is so small, this was not done on Hermes.

A different concern about pulse jitter or mistiming arises when the IVA bore uses magnetic insulation, which assumes ideal pulse timing and uniform current feed at all points on the bore circumference. For example, in Hermes-III each cell is driven by four pfls. These are charged by four different intermediate stores whose output switches are all triggered at the same time. The four pfls connect to the cell at points $90^{\circ}$ apart. To avoid jitter between pfls resulting in a nonuniform drive of the bore, a system of azimuthal oil transmission lines mixes the four pulses and generates four new pulses that were the time average of the original four. These equal, averaged pulses were then delivered to four points $90^{\circ}$ apart on the vacuum cell insulator. A similar oil line system used on Helia, which had two pfls per cell, was outlined in Sec. III C 1.

Oil line pulse-mixing features like those in Helia and Hermes-III not only suppress the effects of pfl jitter on azimuthal uniformity within the cell, they also reduce the effective jitter of the cell drive by averaging the timing of some number of pfls. In Hermes-III, with four pfls per cell, the effective jitter of the drive of a cell is reduced by (4) $)^{1 / 2}$ or 2 , from the $3-4 \mathrm{~ns}$ of a pfl switch to $1.5-2 \mathrm{~ns}$. The mistimings that result from driving successive cells with intermediate switches fired at a single time is of course not reduced.

In RITS [28], there is only one pfl per cell, and thus there is no averaging of jitter. PIC simulations have indicated that in the case of RITS mistimings with $\sigma \sim$ 4.5 ns with $10 \%-90 \%$ rise time of $\sim 20$ ns cause a reduction in load energy delivered on a $70 \mathrm{~ns}$ pulse of about $10 \%$. Some of the reduction is due to magnetic insulation failure and some to the load energy reduction that inevitably results from the increase of rise time and loss of pulse flattop due to the jitter.

Another effect of mistimings of the drives of individual cells arises because the cells are in a series. As discussed in Sec. IIIB1, the driver of a cell somewhere in the middle of a long adder, even if that adder is matched to the driver, "sees" high bore impedances both upstream and downstream and by itself will develop across the cell a voltage near its open-circuit value. The (ideally) simultaneous arrival at this cell of the pulse in the bore that is formed by adding the drive voltages of all the cells upstream delivers a reverse voltage to the cell and reduces the cell voltage to the matched driver value. However, if the drive of a cell occurs too early, before the drive from the adder upstream arrives at the cell, the near opencircuit voltage can be developed in the intervening time. If the cell drive occurs late, the voltage produced at the cell by the other drivers coupled through the adder causes a reverse voltage to develop across that cell until it receives its drive pulse.

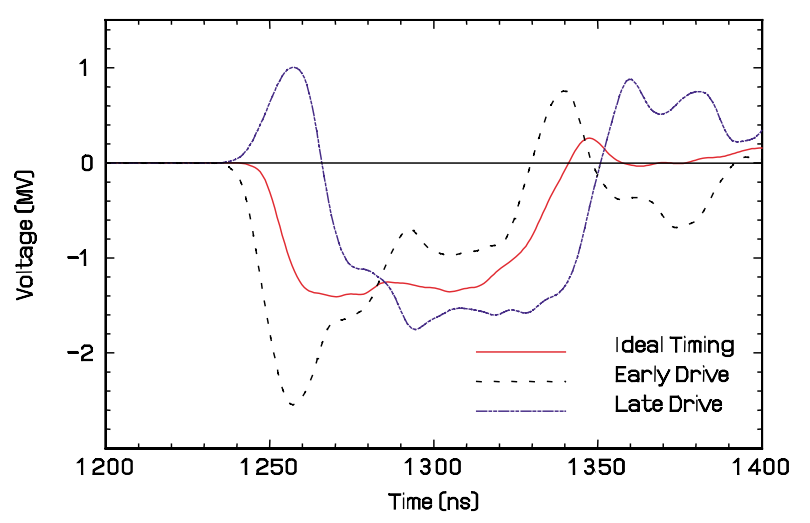

FIG. 29. (Color) Insulator voltage waveforms for ideal switch timings and 95th percentile worst-case timing for early and late driven cells.

In designing RITS, which has one pfl per cell and so no averaging of $\mathrm{pfl}$ jitter, the statistics of such timing-error induced voltages was studied in detail, and Fig. 29 (from Ref. [28]) shows some extreme cases. The RITS cells were designed to withstand these nearly worst-case waveforms, although insulation criteria for the bipolar cell waveform that results from a late drive of that cell are uncertain, particularly in the case of the vacuum insulator surface, which is designed for a particular polarity, and thus may be particularly vulnerable to bipolar voltage waveforms. Early results from the three-cell version of RITS do not indicate problems due to these waveform anomalies, at least with a rise time that is somewhat longer than planned because less sharpening is employed (see Sec. III D 2), so that the waveform anomalies are smaller than indicated in Fig. 29.

In the case where the number of pfls and cells is equal, some averaging of pfl timing and consequent reduction of the waveform anomalies would be possible by driving two cells in parallel on opposite sides with a pair of pfls. This would also help obtain an azimuthally uniform drive to the magnetically insulated bore. This feature was not included on RITS because it would have made reconnection into the various one- and two-pulse versions difficult.

Reducing the jitter of the driving sources, e.g., by using triggered instead of self-closing output switches, can eliminate or greatly reduce concerns about effects of mistimings on cell voltages and magnetic insulation. Simple pulse forming lines or Blumleins with laser triggered or electrically triggered gas switches rather than self-closing water switches have been used in other applications, and the Blumleins have been used in some IVAs. Such switches can have jitters in the $1 \mathrm{~ns}$ range, which essentially eliminates concerns for anomalous cell voltages or for magnetic insulation failures. Such switches also have the advantage that because their jitter is not a function of charge time they can be charged directly from a Marx instead of needing a low-inductance intermediate circuit. Simple pfls with 
laser-triggered output gas switches result in compact IVA designs with flexible packaging [57] and in fact may be used in the AWE Hydrodynamic Radiographic Facility design instead of Blumleins.

Simple pfls with triggered output switches have not been used in the example IVAs presented here. A Blumlein with laser-triggered output switches is used in the AWE IVA described [34]. As we have noted (Sec. IIIC) use of a Blumlein instead of a simple pfl can be helpful in resetting cell cores. However, it introduces a new effect. If a number of Blumleins driving different cells are charged through parallel connections, the Blumlein switches, because they are closed at different times in order to provide the right timing of IVA pulses, interact with one another after closure. The interaction occurs because of the inductive voltages that are still present during the pulse rise time. The result of the interaction is that the Blumlein output pulse waveforms differ from Blumlein to Blumlein and hence from cell to cell. These differences are magnified by the series connections in the adder. They can be minimized by a careful layout of the Blumlein charging connections.

The effect just described for Blumleins is absent when simple pulse forming lines are used, because charging connections to the pfls are transit time isolated from the pfl output pulses.

\section{Prepulse}

Some voltage will be present at the IVA load diode before the main pulse, chiefly the voltage that is coupled through the adder as the pfls charge (in tenths of microseconds to microseconds). This "prepulse" voltage is an important consideration because it can cause unwanted effects in the diode, such as the generation of plasma that changes the effective geometry of the electrodes or even shorts the diode. Therefore the design of the IVA drivers

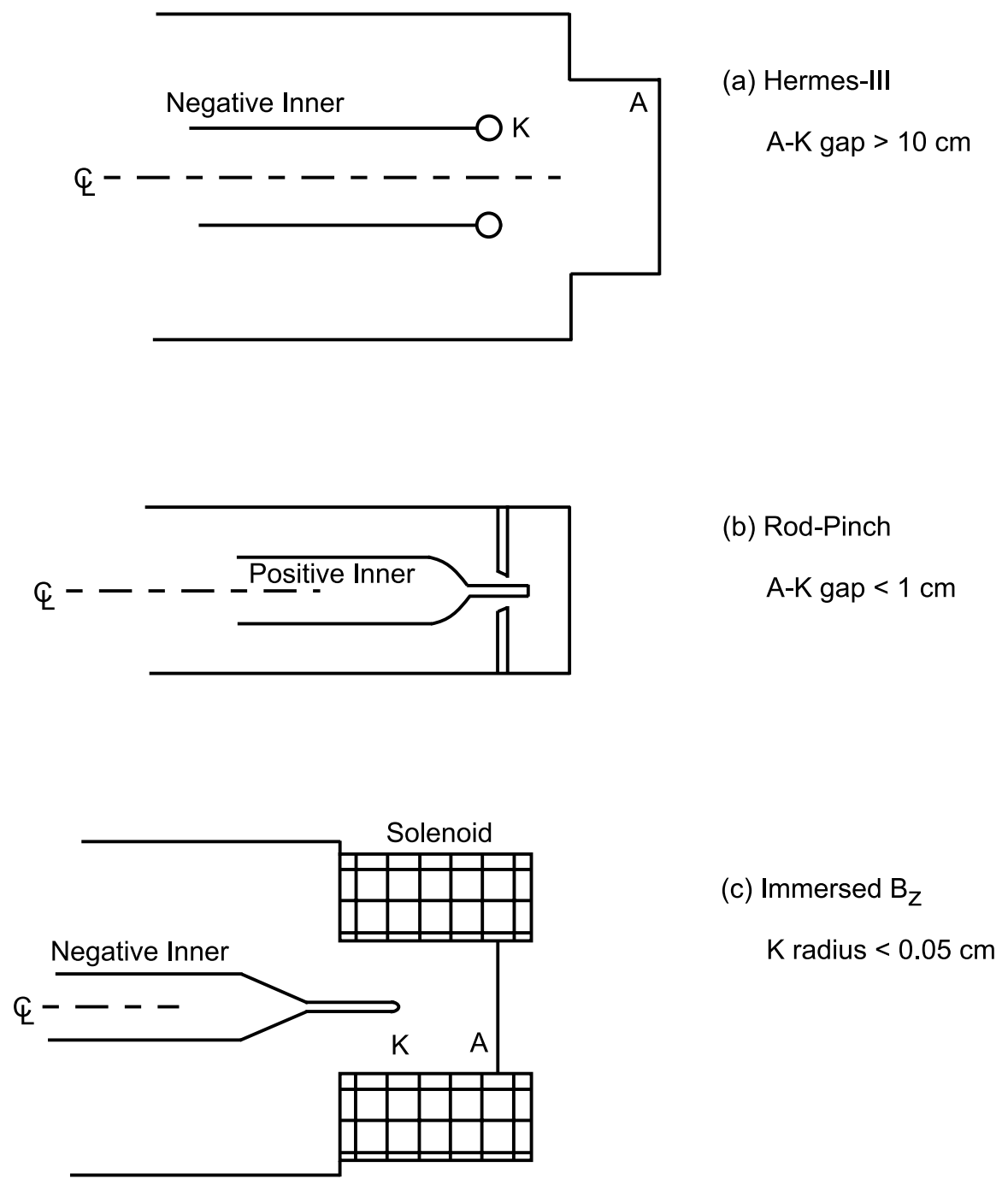

FIG. 30. Diodes with very different sensitivity to prepulse voltages. (a) Hermes-III $A-K$ gap $>10 \mathrm{~cm}$. (b) Rod-pinch $A-K$ gap $<1 \mathrm{~cm}$. (c) Immersed $B_{z} K$ radius $<0.05 \mathrm{~cm}$. 
must often meet the criterion that the prepulse they produce at the diode is less than some value.

The magnitude and polarity of the prepulse allowed are a function of the diode design. Figure 30 illustrates this for several diodes of interest. The Hermes-III diode, Fig. 30(a) has large electrode spacing and only moderate field enhancement; it can withstand prepulses of hundreds of kilovolts of either polarity. The rod-pinch diode, Fig. 30(b), has a large field enhancement on the positive (rod) electrode but may have little on the negative. It will be affected by prepulses of tens of kilovolts of opposite polarity to the drive pulse but may withstand larger prepulses provided they are the same polarity as the drive pulse (which to date is usually positive). The immersed diode, one of the diodes planned to be used on RITS, Fig. 30(c), has a cathode with radius $r \sim 0.25 \mathrm{~mm}$. The electric field at its tip under a voltage $V$ is roughly $V / 1.5 r$, and to keep this below an electron emission threshold of $\sim 150 \mathrm{kV} / \mathrm{cm}$ before the main pulse the voltage must be less than about $5 \mathrm{kV}$ when it is negative. Much larger positive prepulse voltages (opposite polarity to the drive pulse in this case) can be tolerated.

A driver consisting of a simple pulse forming line with series output switch will produce a prepulse voltage of the same polarity as the output pulse by capacitive coupling of the pfl charging voltage through the output switch. This prepulse may be reduced before it reaches the IVA load by putting one or two more switches in series. Because it will be used to drive the prepulse-sensitive immersed diode, the RITS pfl, Fig. 31, has two more switches in addition to the main output switch of the $\sim 2.8 \mathrm{MV}$ pfl. One of these is for the purpose of sharpening (reducing) the rise time, as well as reducing prepulse. Like the pfl output switch, it is in water, but its series coupling capacitance is reduced by a ground plane shield. The other switch, which is solely for prepulse reduction, is in oil and therefore has a very small capacitance. At a pfl maximum charge voltage approaching $3 \mathrm{MV}$, the prepulse of the RITS line with its two prepulse switches is estimated in circuit simulations to be about $0.5 \mathrm{kV}$, and in a 12-cell RITS the diode prepulse is similarly estimated as around $5 \mathrm{kV}$.

Because two prepulse switches are used in RITS, the prepulse is bipolar and oscillatory. The RITS prepulse amplitude is also somewhat unpredictable, for two reasons. First, it is controlled by initial pfl charging transients. These are produced by the closure of the intermediate store gas switch that charges the pfl. This is a rimfire switch [58] with many series sections and the successive closure of these is hard to model in simulations. Second, the response of the Metglas to low-level oscillatory pulses in reducing or changing the prepulse is hard to predict. In the event that the prepulse is too large in practice, one RITS cell can be driven through an inductor with a bias voltage that ensures that the prepulse is only positive at the diode and so does not affect an immersed diode load.

pfls similar to those of RITS but without any switch other than the pfl switch have been used successfully on Sabre to drive rod-pinch diodes [59]. This is because while with only the pfl switch the prepulse is much larger, it is unipolar and has the polarity of the output pulse, which, as noted above, the rod pinch can tolerate.

Hermes-III used only the water prepulse switch, because of the insensitivity of its large area diode to prepulse. The water prepulse switch also served as a sharpening switch, reducing a $\sim 20 \mathrm{~ns}$ rise time from a self-closing water output switch to $\sim 10 \mathrm{~ns}$, and this sharpening was an important function on Hermes.

We have noted (Sec. III C) that when a Blumlein is used to drive the IVA cells the charging current for its floating or inner line may be used to reset the cell cores more completely, from $-B_{\text {rem }}$ to $-B_{\text {sat }}$. The voltages developed across the cell cores in this process produce prepulses at the cells. These add to produce a prepulse at the diode, one that initially has polarity opposite to the output pulse but which becomes bipolar later in time after the cell cores saturate. This prepulse can be reduced by increasing the Blumlein charge time relative to the output pulse duration. It is a function of the flux density swing that the cores need to go from remanence to backsaturation; for Metglas $2605 \mathrm{CO}$, the most common IVA core material, $\left(B_{\text {sat }}-\right.$ $\left.B_{\text {rem }}\right) \sim 0.05 \times \Delta B\left(\Delta B \approx 2 B_{\text {sat }}\right)$. The prepulse is also a function of core losses; loss currents can help charge the inner Blumlein line and reduce prepulse.

To calculate the prepulse waveform in the case of the Blumlein is not trivial; it requires a dynamic timedependent model of the cores. For the AWE IVA prototype cell and Blumlein [60], the diode prepulse developed in resetting the cores to $-B_{\text {sat }}$ was estimated by simulations that included the PSD core model to be about $8 \%$ of the final pulse voltage, and these simulations were

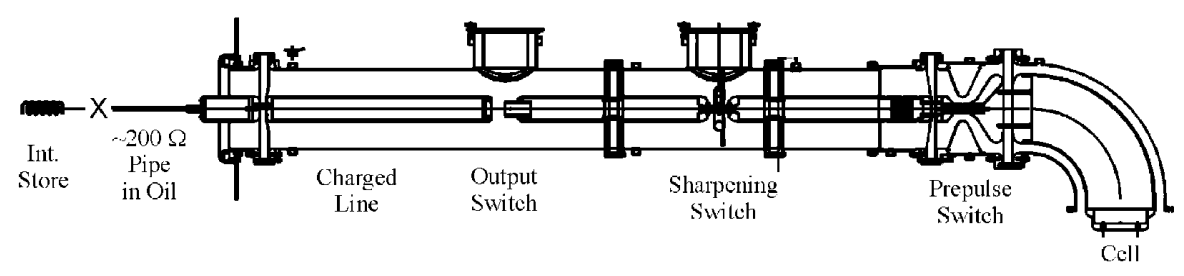

FIG. 31. The $7.8 \Omega$ RITS pulse forming line. 
approximately verified by a single-cell prototype test. A method for suppressing this prepulse further was investigated, namely, actively reverse saturating the cells before Blumlein charge began. It was estimated that the finite inductances of the cells with saturated cores would develop prepulses adding at the diode to $1.5 \%-2 \%$ of the output pulse. This was a substantial improvement, but was still more prepulse than desired, and a series prepulse gas switch was therefore introduced between each Blumlein and the cell it drives, along with an inductor upstream of the switch to charge the Blumlein inner line. The cell cores then received only a dc reset, to remanence. The prepulse is estimated in simulations to be reduced to the order of $0.5 \%$ of the output pulse.

It is also possible to reduce diode prepulse by introducing a flashover switch into the conductor feeding the diode in vacuum, as has been done on pulse systems other than IVAs. In an IVA, the optimum position for such a switch is just downstream of the last cell, so that no cell couples prepulse to the inner (stalk) conductor beyond the prepulse switch. This technique was successfully used in tests of the immersed diode on Sabre and Hermes-III, [61]. It can reduce prepulse by a factor of a few or so up to about 10, depending on the geometry. The effect of the flashover switch on magnetic insulation and on power flow downstream may need to be considered, however.

The best solution to reducing prepulse in IVAs is to use pfls that are slowly pulse charged and have low capacitance output switches such as gas switches. Such slowcharged switches must be triggered to obtain adequate $\mathrm{pfl}$ timing.

\section{EXAMPLE IVAS: PAST, PRESENT, AND FUTURE}

In this section we give examples of actual IVAs. Section IVA is devoted to Hermes-III, the largest IVA to date, designed for negative output using magnetic insulation. Section IV B is devoted to Cygnus, a positive output IVA that works below electron emission threshold rather than use magnetic insulation and which incorporates voltage transformation by using a common source to drive three adder cells.

Section IV C more briefly discusses a number of other IVAs, indicating and discussing their features of interest, and, in particular, features not seen in Hermes-III or Cygnus. The reader is provided references where these IVAs are described in more detail.

\section{A. HERMES-III}

Hermes-III at $20 \mathrm{MV}$ (negative), $750 \mathrm{kA}, 40 \mathrm{~ns}$ (Fig. 32, Refs. [37,52]) is the largest and most powerful IVA built to date. Initiated (by Sandia with PSI design support) immediately after the Helia demonstration of voltage addition in magnetic insulation at $4 \mathrm{MV}$ [26], it was a bold extrapolation from Helia in terms of voltage and number of cells. Its design made provision for lengthening the drive pulse in case a voltage addition in the magnetic insulation took time to establish, but this provision proved unnecessary.

The vacuum bore through the twenty $1 \mathrm{MV}$ cells of Hermes III is $76 \mathrm{~cm}$ in diameter and $12.8 \mathrm{~m}$ long. The cathode stalk is fabricated in twenty cylindrical section steps to achieve the impedance prescribed according to Sec. III B 2. It then extends a further $4.6 \mathrm{~m}$ beyond the twentieth cell to the diode in a constant impedance output MITL, forming a total stalk cantilever length of $\sim 17 \mathrm{~m}$. This is illustrated in Fig. 33, in which some details of the cell can also be seen. To maintain the straightness of the stalk in each cell, it is fabricated in seven sections, and these are joined at flanges that incorporate bevel rings that allow the downstream section to be angled up by a small, controlled and variable angle, offsetting droop due to gravity accurately on average [62]. A practical rule of keeping the spacing between the stalk and the outer of the bore to within $5 \%$ or $10 \%$ of its ideal value has proved

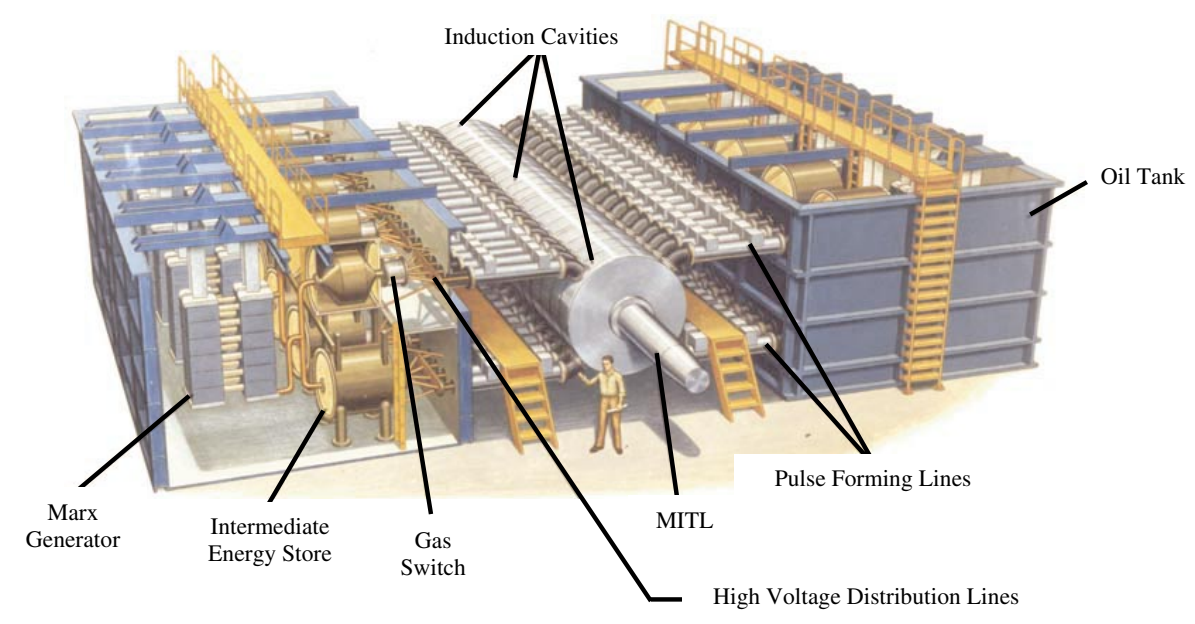

FIG. 32. (Color) Hermes-III showing details of Marxes and intermediate stores. 


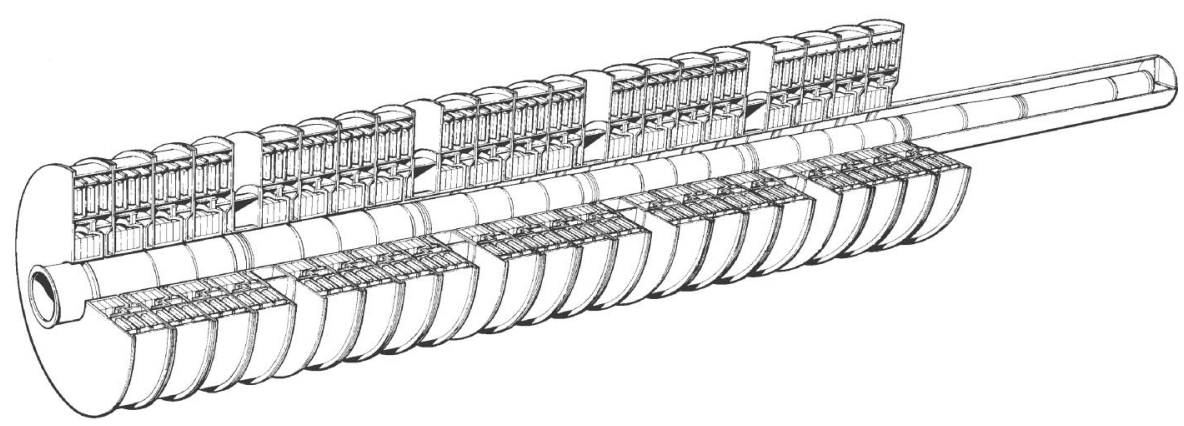

FIG. 33. Cutaway of Hermes-III IVA, showing vacuum power flow region, stalk, and some cell details.

successful in Hermes and in other IVAs. The inner of the output MITL is prebent upwards to compensate gravity droop.

The length of Hermes-III gives it a vacuum double transit time of $>100 \mathrm{~ns}$, and many cells do not "see" the load diode during the $40 \mathrm{~ns}$ pulse. The magnetic insulation wave travels towards the diode slightly slower than light in vacuum, and a precursor signal that propagates at light speed before electron emission from the stalk turns on and therefore arrives at the Hermes-III diode a few ns before the main pulse, Fig. 34.

The cells are $\sim 3 \mathrm{~m}$ in diameter, Fig. 35 . Their induction cavities contain Metglas 2605CO cores with inner diameter $90 \mathrm{~cm}$ and build $20 \mathrm{~cm}$. A $\sim 1.5 \mathrm{~m}$ diameter multistage Rexolite vacuum insulator stack surrounds a radial vacuum feed designed at $200-250 \mathrm{kV} / \mathrm{cm}$. Electron emission in the radial vacuum feed is suppressed by using an anodized aluminum surface as the negative conductor. The anodization extends slightly around the inner corner of the cell into the bore. To achieve $750 \mathrm{kA}$ at $1 \mathrm{MV}$ per

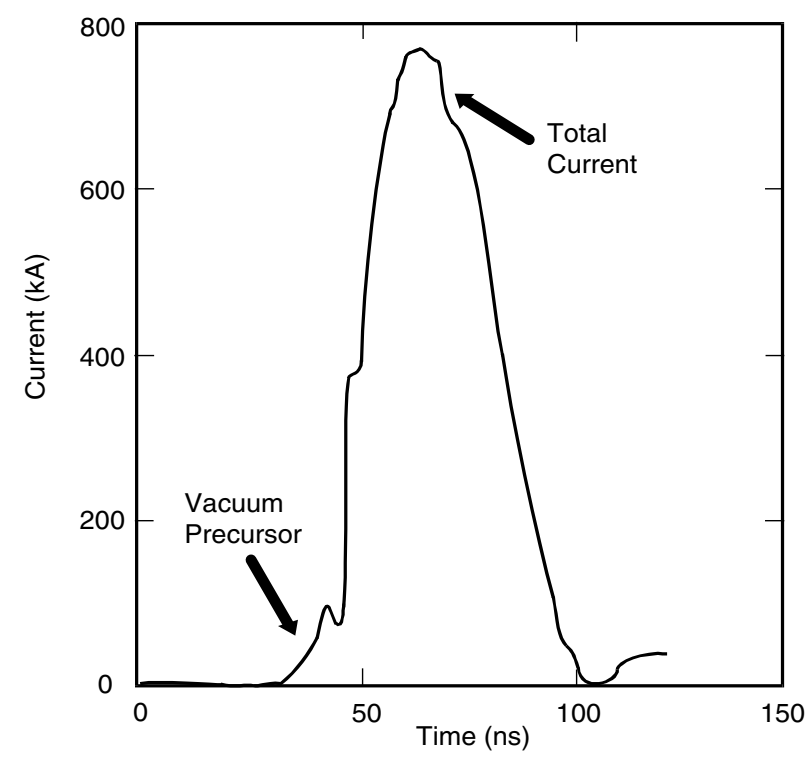

FIG. 34. Hermes-III output current (showing vacuum precursor) (from Ref. [54]). cell, four $5 \Omega$ pfls drive each cell at four points $45^{\circ}$ each side of top center and bottom center. Inside the cell, the pulses travel in a system of azimuthal triplate oil lines with their electrode widths vertical. These lines mix the four pulses and then drive the vacuum insulator stack at four equidistant points with identical and simultaneous pulses.

The Hermes-III pfls, of which there are 80 , are an earlier version of the RITS pfl illustrated in Fig. 31. The $\sim 2.4 \mathrm{MV}$ charged line has a constant $5 \Omega$ impedance and is switched by a self-closing water spark to a $5 \Omega$ output line that includes a second water switch that sharpens the rise time to about $10 \mathrm{~ns}$ and reduces the prepulse. The oil prepulse switch used in RITS is unnecessary because the Hermes large area diode is not affected by the prepulse of about $100 \mathrm{kV}$.

The pfls are fast charged in about $200 \mathrm{~ns}$ to minimize the jitter of their self-closing output switches, which is $3-$ $4 \mathrm{~ns} \sigma$. The two pfls on either side of each cell are charged from two intermediate stores via 2.4 MV laser-triggered rimfire (multistage) switches [50] that have 1-2 ns jitter. The cell is thus driven by four pfls that have essentially

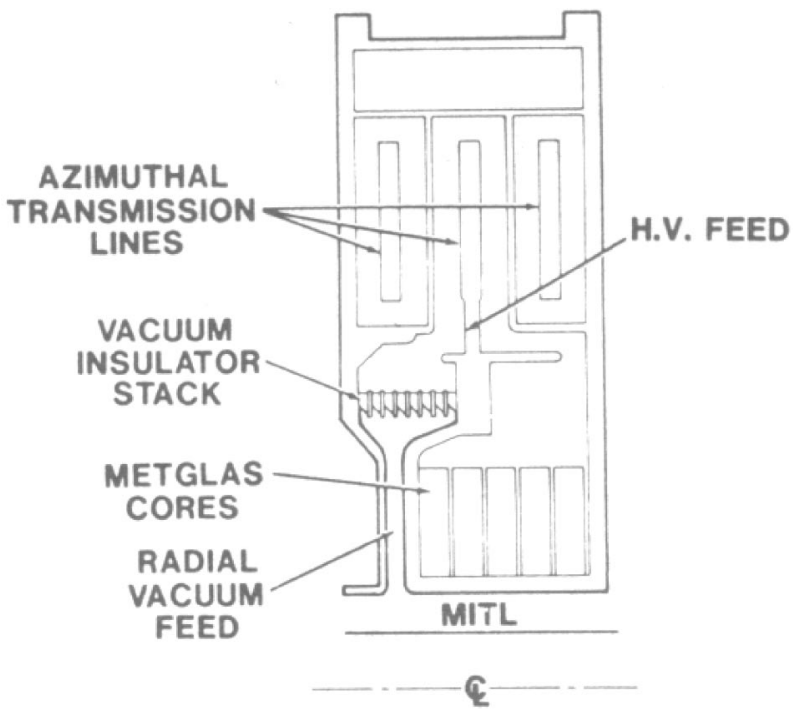

FIG. 35. Hermes-III cell schematic (from Ref. [52]). 
independent timing, and the jitter of the cell drive is thus reduced by $\sim \sqrt{4}=2$ to the range of $1.5-2 \mathrm{~ns}$.

An oil-filled tank on each side of the line of cells (Fig. 32) contains the intermediate stores, arranged in vertical pairs, and the rimfire switches. Each store and switch charge four pfls arranged in a horizontal line that drive four successive cells at the same azimuthal locations. The pfls are in air, and therefore access to the two switches in each pfl for adjustment or refurbishment is easily obtained from ports above the switches. Both switches have lifetimes of thousands of shots, operating after a while with their active surfaces completely roughened by arc pits. The pfl (first) switch has a maximum gap of $6-7 \mathrm{~cm}$ and closure in the water is initiated by field enhancement at a ring electrode about $8 \mathrm{~cm}$ in diameter on the downstream (positive) side. Although there are reports of more pits produced on the electrodes by breakdown than the number of shots, the $\sim 2$ ns transit time across the switch electrodes and the $\sigma \sim 4$ ns jitter presumably mean that usually only one channel will form for each pulse. Based on recent results with the similar Cygnus pfl and with the water switches in the PSD PITHON generator, about $30 \mathrm{kV} / \mathrm{cm}$ or $\sim 200 \mathrm{kV}$ will remain across the water switch arc after closure, representing a $\sim 20 \%$ power loss compared with an ideal switch. During ringing of residual energy after the pulse, this voltage drop probably helps other late channels to close.

The rise time from the Hermes-III pfl switch is $\leq$ $20 \mathrm{~ns}$, with comparable inductive and resistive phase terms, and this is reduced to $\sim 10$ ns by the second water sharpening switch. This has a similar construction to the pfl switch, but smaller diameter electrodes set $\leq 1 \mathrm{~cm}$ apart. The sharpening switch closes with negligible jitter when the voltage has risen to near full value. In addition to sharpening the rise, this switch reduces prepulse, and to maximize this reduction (as in the RITS pfl, Fig. 31) a ground plane extends across most of the line diameter to reduce the capacitive coupling between the inner conductors. The sharpening switch closes through an aperture in this ground plane, and the size of the aperture can be varied by replacing the central part of the plate to effect a controlled closure from inner to ground late in the pulse, in order to control pulse duration or speed the fall of the pulse. This function is known as crowbarring; it is also useful in decoupling residual driver energy from the cell, the bore, and the diode.

The Hermes-III pulse power system is completed by ten 2.4 MV Marx generators. There are five in each of the two oil tanks, each charging two intermediate stores.

Hermes-III diagnostics include $D$-dot monitors to record voltages at many points in the water dielectrics and in the oil of the cells. Current monitors around the outer and the inner of the vacuum voltage adder are $B$ dots that can be recorded individually to observe azimuthal symmetry or summed to record total current. Any differences in the outer currents along the machine would indicate loss currents flowing from the stalk to ground, but in negative polarity these losses are negligible. Excess of the inner over the outer current at a given axial location indicates the fraction of the current that flows in the form of vacuum electrons, which according to the theory presented in Sec. III B 2 is two-thirds of the total current at 20 MV. As described near the end of Sec. III B 2, the theory of Ref. [36] allows the voltage in the output MITL near the diode to be calculated from the currents in the inner and outer; the voltage so inferred is confirmed by ion range and bremsstrahlung dose measurements. The electron flow in vacuum arrives at the diode region and there travels to the final anode, participating fully in the desired bremsstrahlung production.

Hermes-III became operational in 1988 and can fire dozens of shots per day. It has been very reliable. It was briefly tested in positive polarity after turning each cell through $180^{\circ}$, still using the stalk designed for negative polarity [39]. It was found (as expected) that electrons are emitted from the outer of the bore between cells and cross radially to arrive at the stalk with energies that vary about linearly along the length. The total loss current was about $30 \%$ of the total current; because of the distributed energy of the loss electrons the energy loss was only about $15 \%$. Loss current loads only the cells upstream from where it is emitted from the outer. Therefore in positive polarity loading on the pulse power decreases towards the load, and downstream cells develop more voltage than upstream cells. The voltage developed across the load in this test, a reverse electron diode, was about $21 \mathrm{MV}$.

\section{B. Cygnus, a positive adder}

The two nearly identical $+2.25 \mathrm{MV}$ Cygnus I and Cygnus II IVA systems [3] are designed to operate together in a limited space in a tunnel. Space limitations make it necessary to divide each system into two parts: (1) the adder cells and diode they feed, and (2) the pulse forming line and its Marx charging system. The layout of Cygnus I, which has recently been tested successfully at the design level at Los Alamos [63] is seen in Fig. 36. There is a single $7.8 \Omega \mathrm{pfl}$, similar to that of RITS [Fig. 12], except for (1) a slightly shorter pulse and (2) a profiled wave impedance in the charge line that is designed to ramp the output pulse and compensate for a load impedance that falls with time. Charging a single pfl, a low inductance Marx can achieve a charge time approaching the speed achieved using intermediate stores to charge four pfls in Hermes-III (see Sec. IV B) or three in RITS; therefore without the need for intermediate storage the design achieves an adequate safety factor in pfl insulation and adequate self-closing switch performance.

The pfl output is connected to the adder some distance away by a single "water cable." Figure 36 shows that this 


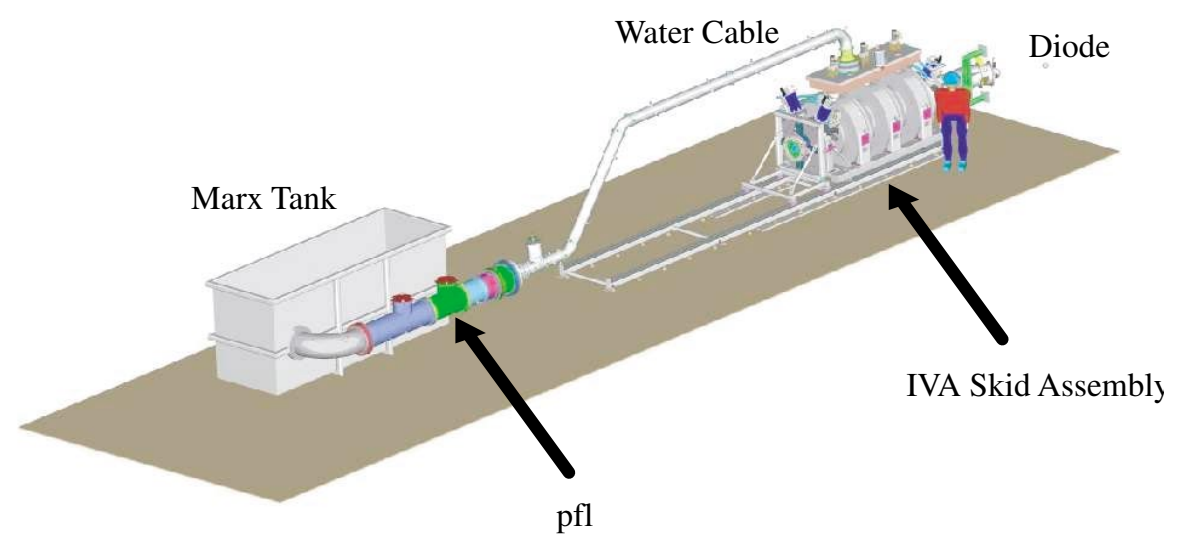

FIG. 36. (Color) Cygnus-I accelerator layout.

rises to a height near the tunnel ceiling that allows it to pass over intervening equipment, then approaches the adder from vertically above. The Cygnus 2 adder will be alongside that of Cygnus 1 and its Marx and pfl will be behind those of Cygnus 2, so that the Cygnus 2 water cable will span an even greater distance.

The water cables are not flexible, but are simple coaxes easily designed to take a desired route. They are passive except for a self-closing water switch diverter near the output of the pfl which is designed (using the polarity sensitivity of water breakdown) to pass the negative drive pulse and then break down to protect the pfl by absorbing energy if the diode driven by the adder should shortcircuit or the adder cores not be reset; either occurrence would result in a large reverse pulse being reflected toward the pfl. The water cables are fabricated in sections whose impedances decrease in the direction of the adder, transforming the pfl output voltage down somewhat and increasing the pfl current from a $\sim 170 \mathrm{kA}$ matched output to the $\gtrsim 250 \mathrm{kA}$ needed to drive the three-cell adder with up to $80 \mathrm{kA}$ per cell including a few $\mathrm{kA}$ core loss current.

The drive from the water cable splits into three at an oil manifold above the adder cells. The manifold is designed to achieve approximate impedance matching and approximately correct timing in its drive of the individual cells and was optimized by iterative computer simulation. Through the manifold, three plungers allow the reset of the Metglas cores in each cell in turn. The plungers both make the connection to the cell being reset and the disconnect from the manifold connection to the other cells. This is preferable to resetting all three cores simultaneously in parallel, because at low voltage contact resistances could result in the reset current dividing unequally between cells and not completely resetting one or two cells.

The three-cell Cygnus adder is shown in Fig. 37. Note that when negative pulses (the easiest polarity for the pulse power to deliver) are used to drive a positive adder, the radial feed in the first cell is at the upstream end, making in effect one more adder bore section than would be present in a negative adder.

To provide the three cells needed in each of the two Cygnus IVAs, cells were taken from the now-dismantled Sabre IVA [40] and were refurbished and modified. The modifications included reducing the number of feedpoints from two (one at the top and one at the bottom) to one at the top. This made a more packageable

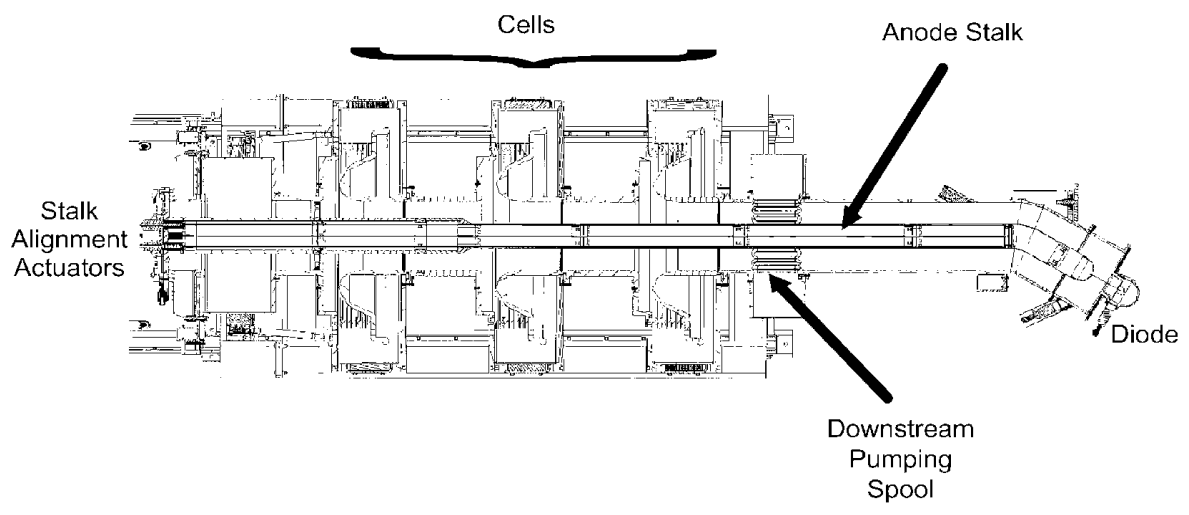

FIG. 37. Cygnus IVA cross section. 
design and was possible because the Cygnus adder bore operates below electron emission threshold rather than magnetically insulated, so uniformity of current feed to the bore is unimportant. The single-point feed is made direct to the rim of the vacuum insulator stack with no azimuthal oil line (compared with the RITS cell in Fig. 21), and this adds a few ns to the rise time. The diode voltage in Cygnus is $2.25 \mathrm{MV}$, or $0.75 \mathrm{MV}$ per cell, but inductive drop along the adder bore and output MITL causes each cell to experience a voltage of about $1 \mathrm{MV}$, similar to that in Sabre. The limitation imposed by the area and hence volt-second product of the four Sabre 2605CO Metglas cores is one reason that a $\sim 5 \mathrm{~ns}$ pulse length reduction was made in adapting the RITS pfl to Cygnus.

Because it is driven at a single point with $\sim 1 \mathrm{MV}$ and $\geq 250 \mathrm{kA}$ and delivers $\sim 3 \mathrm{MV}$ and $\sim 80 \mathrm{kA}$ to the output MITL, the adder manifold can be regarded as a true 3:1 voltage transformer.

In order to minimize electron emission from negative surfaces in the vacuum, clear hard anodizing is applied to any negative surface on which the field is greater than about $100 \mathrm{kV} / \mathrm{cm}$, and the field on such surfaces is limited to about $200 \mathrm{kV} / \mathrm{cm}$. These are conservative stresses based on Ref. [42] and experience with HELIA and Hermes-III. Meeting the $200 \mathrm{kV} / \mathrm{cm}$ criterion required new radial vacuum feeds for the cells obtained from Sabre. A step was introduced on the anode side of the radial vacuum feed in order to increase spacing from the cathode plate, at the same time allowing the radial part of the anode feed plate to slide away and expose the insulator for easy cleaning, a technique used for the first time in Cygnus. The anodized cathode feed plate was remade with a larger corner radius at the entrance to the bore, where the electric field is enhanced. Anodization is not terminated after passing around this corner, as it is in negative adders, because in this case the electric field does not change sign; instead the anodization continues through the bore of the each cell. Anodization is also applied to the inside surfaces of the spools that separate the cells and provide cleaning access, to the pumping slots in the downstream pumping spool, which are formed by specially shaping the spool surface to minimize field enhancement, using numerical machining, and to the inside of the $\sim 2 \mathrm{~m}$ vacuum coax that connects the adder to the diode.

The impedance of the rod-pinch diode used on Cygnus falls from about 55 to $25-30 \Omega$ during the $\mathrm{x}$-ray producing part of the power pulse, with an average in the $\bar{Z} \approx$ $35 \Omega$ range. The coaxial sections of the adder, which function as electron-free vacuum transmission lines, are designed with rather higher wave impedance than would be ideal for this load $(\bar{Z} / 3,2 \bar{Z} / 3, \bar{Z})$, because this reduces the field on the anodized outer coax. Computer simulations show that the diode power waveform is not much degraded by some degree of mismatch. The coax imped- ance is $40 \Omega$ in the first cell then $60 \Omega$ through the second and third cells and to the diode. The mismatch does lead to some inductive drop along the adder (mentioned earlier), especially with the falling diode impedance. With a 2.25 MV peak at the diode, design simulations showed about 2.9 MV across the vacuum coax just downstream of the third cell.

The Cygnus accelerators drive rod-pinch diodes angled at $30^{\circ}$ to the IVA axes in opposite directions, so that the radiographic axes defined by the rod directions are at $60^{\circ}$ to one another. The $0.75 \mathrm{~mm}$ rod anode must be placed accurately in the center of a $\sim 10 \mathrm{~mm}$ aperture in the cathode. For this purpose the inner stalk of the adder that drives the rod is mounted on a plate at the rear of the adder that can be deformed to control the direction of the stalk, while tension cables threading the stalk from this region to the angle near the diode accurately adjust the location of the tip. All adjustments are made with the bore and diode under vacuum, while the diode is viewed through ports nearby.

The first tests of Cygnus I at Los Alamos [63] have selected a rod-pinch diode configuration believed to be optimal. Voltage and current waveforms are shown in Fig. 38. These were taken at $\pm 40 \mathrm{kV}$ charge on the Marx, which is rated at $\pm 50 \mathrm{kV}$.

The sensitivity of the Cygnus rod-pinch diode to prepulse has not been documented by experience, but can be estimated as was done in Sec. III D2. An unwanted,
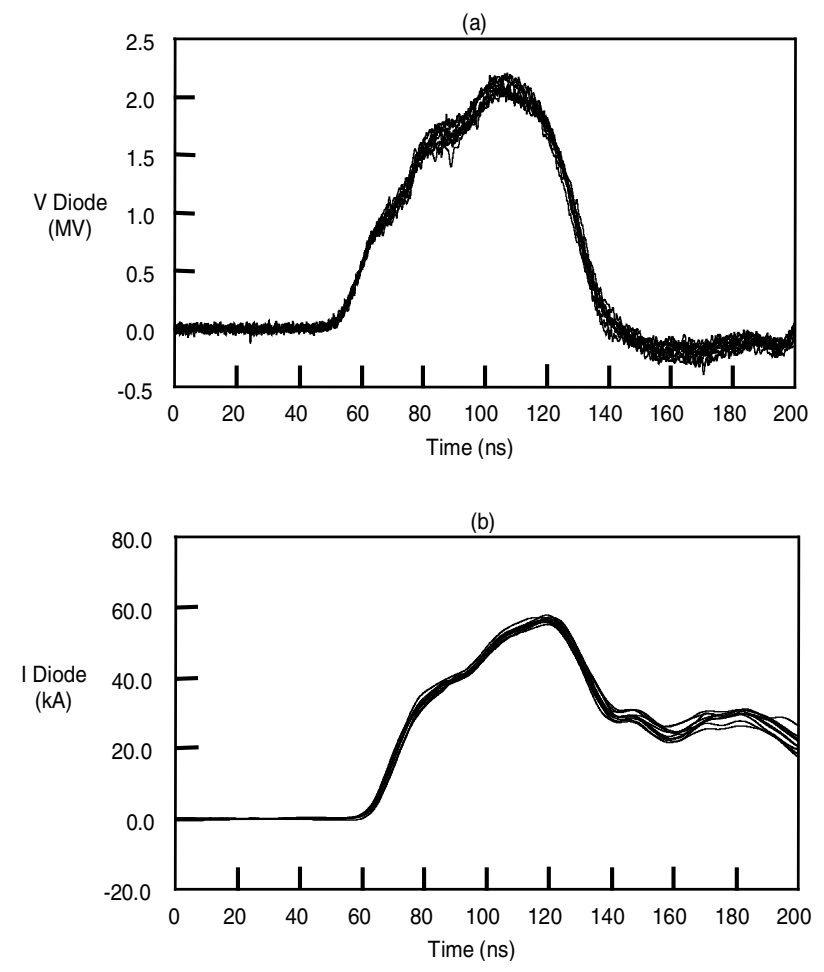

FIG. 38. Ten shot overlay of Cygnus diode (a) voltage and (b) current. 
nonuniform generation of plasma at the rod may occur at output voltages of the order of $-20 \mathrm{kV}$, and at the cathode edge, which in this case is somewhat sharp, at positive output voltages possibly somewhat higher. Use of the oil prepulse switch originally designed for RITS results in a few hundred volt prepulse downstream of the switch, and probably about $1 \mathrm{kV}$ or less at the diode, which is very safe.

\section{Other IVAs}

Sections IVA and IV B have illustrated in some detail examples of a negative adder that uses magnetic insulation to avoid electron loss (Hermes-III) and a positive adder that uses suppression of electron emission and achieves 3:1 voltage transformation. In this section we mention other IVAs that have been built and are under development, and we point out where these have features of interest that are different from Hermes-III and Cygnus. References are provided where more details can be obtained.

Features of RITS [28] have been used throughout this article as illustrations. The RITS design was intended to provide a twelve-cell, $16 \mathrm{MV}, 150 \mathrm{kA}$ IVA, and its novel feature of a single-point cell feed with an azimuthal oil line within the cell to distribute current to the magnetically insulated bore was validated recently at the level of three cells and $4 \mathrm{MV}$. More elaborate diagnoses have been made on RITS than in previous IVAs of bound and sheath current distributions in the bore. These have shown some interesting phenomena that have not resulted in other than ideal output characteristics, but which could affect the performance of versions with more cells and higher voltage. It was also intended that the RITS design be adaptable to deliver two pulses to a single diode at intervals in the range of microseconds, using an isolation network with a blocking core and a polarity sensitive gas switch to control the drive of each cell from two pfls [28]. The two-pulse design has yet to be explored in detail.

AWE plans an IVA similar to RITS (14 MV, $150 \mathrm{kA}$, $70 \mathrm{~ns}$ ) [34]. Unlike RITS, which is to be a test bed that can be reconfigured in various ways, the AWE IVA must fit on a platform of limited size. AWE planned the use of waterdielectric Blumlein pfls. Experience was gained in the prototype of this IVA (PIM) and in an earlier, smaller IVA (LYNX) [64]. In these IVAs the charge of the Blumleins was used to reset cell cores. Breakdown between the outer turns of cell cores led AWE to provide radiused shields for these regions.

Sabre [40] was a positive-output IVA that incorporated the four $1 \mathrm{MV}$ Helia cells and six additional cells and was used for many years in Sandia's program to develop ion diodes at voltages approaching $10 \mathrm{MV}$. It is responsible for most of the experience with magnetically insulated positive adders. It was found on Sabre that two-point cell feed without azimuthal lines resulted in a drive of the adder bore that was adequately uniform for magnetic insulation.

An IVA approach was selected for Jupiter, a $10 \mathrm{MV}$, $60 \mathrm{MA}, 100 \mathrm{~ns} Z$-pinch system [44] and a prototype cell and driver were built [65]. The cell voltage was increased to $\sim 2.5 \mathrm{MV}$, with a current of $\sim 2 \mathrm{MA}$. A Marx-intermediate store-fast charged pfl pulse power driver was developed that delivered this cell drive using only gas switches; the pfl switches were self-closing. An unusual feature of the Jupiter concept was the idea of using an IVA to drive a $Z$ pinch. In the initial concept, long MITLs and post-hole convolutes were needed to connect the adder cells to the $Z$ pinch, and a concern was that at $\sim 10 \mathrm{MV}$ a MITL operating at $Z_{\min }$ has $\sim 60 \%$ of current in electron sheath flow, which would not be available to the $Z$ pinch. Recent results, including those mentioned in Sec. III B 2, suggest that the unusable sheath flow could be reduced by the choice of MITL impedance. A remaining concern would be the mismatch losses when connecting a long MITL to a load like a $Z$ pinch that has an impedance that varies strongly with time. An alternative for Jupiter-like $Z$-pinch drivers such as $Z X$ [66] is to use similar IVAs but with the bore filled with water instead of vacuum, driving full-voltage vacuum insulator stacks like large versions of those in the Sandia $Z$ accelerator [67].

As noted in Sec. III A, IVAs are more compact than the equivalent oil-dielectric Blumlein, which may be the only alternative technology for some applications. Still, they can be physically large, as illustrated by Hermes-III and by postulated Jupiter or $Z X$ designs based on IVA modules. Their overall dimensions often result from their division into relatively small modules that are readily accessible for maintenance, which is possible because of the distributed nature of the IVA. The distributed nature can be seen as an inherent advantage in a case like that of Cygnus (Sec. IV B), where it enables the IVA to be separated into parts that fit the geometry of a tunnel complex.

Recently work in Russia has addressed the possibility of building IVAs that are very compact by locating the drive circuits with the cells [68]. To make the pulse power drivers small, these use mainly simple dc-charged capacitor circuits. This eliminates intermediate energy storage and switching components; energy can be stored in capacitors at quite high density.

Output pulse durations desired for the Russian IVAs (named Linear Transformer Drivers, LTD, by their designers) are again in the range $50 \mathrm{~ns}$ (for flash radiography) to $100 \mathrm{~ns}$ (for driving $Z$ pinches). It is necessary to divide the drive circuits into many small pulsers in parallel, each with its own spark gap switch, in order to make the inductance low enough to discharge in such short times, even in simple sinusoidal pulses. Shaped (e.g., rectangular) pulses require an even finer subdivision. The addition of passive peaking capacitors, i.e., ones without peaking switches, shortens and squares the pulse 
some. In Ref. [68], 24 parallel discharge circuits are arranged in a circle in a $2.4 \mathrm{~m}$ diameter cell and deliver $500 \mathrm{kA}$ at about $125 \mathrm{kV}$ output. The low cell voltage allows the insulation to be pressurized air or lightly pressurized $\mathrm{SF}_{6}$ rather than oil. The length of the cell is $25 \mathrm{~cm}$.

The synthesis of large energy pulsers from many small simple dc circuits is an interesting approach that was once tried in the early 1980s in the U.S. ("PIMBS," [69]). At a similar output voltage to that of the Russian LTD stage $(125 \mathrm{kV})$, four $\mathrm{SF}_{6}$ insulated modules each with four parallel rail-switched pulsers used peaking circuits to generate 1-1.2 MA for $\sim 70 \mathrm{~ns}$. The four modules drove four specially developed $>1 \mathrm{MA} \mathrm{x}$-ray diodes in parallel rather than being connected in series using cores, but they could easily have been configured as IVA stages. The challenge in the U.S. design was to keep a system with many low inductance circuits operating with many pulses between failures, and this became more difficult as the number of modules increased to four and the number of pulsers to sixteen. In the U.S. this led to the substitution of water lines to drive diodes of the same type. The new Russian approach uses more parallel switches instead of rail switches, with less emphasis on high electric stress to achieve low inductance, and this may enable the complexity/reliability challenge to be overcome.

Both the Russian design and the PIMBS-II design place the pulsers inside metal enclosures, which slows maintenance and further increases the desirable reliability. In order to make the dc pulser components accessible in such an approach, other geometries could be considered. The cell might consist, instead of a closed structure with small feed ports, of a more open structure closed by grounded rods that are spaced widely enough to allow the pulsers to be withdrawn between them for maintenance. The pulsers could also be outside of the cell and feed power in through solid dielectric transmission lines. In this case electromagnetic fields would be present outside the relatively open cell, and a larger, perhaps roomsized shield enclosure might be needed.

The use of simple dc capacitors as IVA stages is also embodied in the "Linear Transformer Drivers" developed at CEG [70], which had Russian antecedents. The French LTDs use $\sim 20 \mathrm{~kJ}, \sim 100 \mathrm{kV}$ capacitors, one or two in each stage, with up to ten stages in the adder, which uses vacuum insulation in the bore. Only pressurized air is used in the region of the capacitor bushings, cores, and feed to the vacuum insulator, and thus oil and $\mathrm{SF}_{6}$ are both avoided. Because fewer and larger-energy parallel circuits are used than in the Russian LTD, the discharge time is much longer, of order $0.7 \mu \mathrm{s}$. This allows cheaper, nonamorphous core materials to be used rather than Metglas. However, the application envisioned, the fast $Z$ pinch, still may require a drive pulse in the $100 \mathrm{~ns}$ range. Therefore the LTD is used to energize an inductor with current (the adder bore can form part of this inductor) and then either an opening switch or a flux-compression circuit is required to act as a power-multiplication circuit in vacuum to drive the $Z$ pinch. If the development of such a power-multiplication circuit is successful, the combination of IVA-LTD technology and inductive storage would provide a cost-effective, oil, and $\mathrm{SF}_{6}$ free pulse power system in which the dc-switching and energy storage components requiring maintenance are easily accessible.

The CEG LTDs develop less than $1 \mathrm{MV}$ during the charge of the energy-storing inductance of the vacuum region. If large power gain is then achieved in a subsequent pulse compression circuit, the voltage on the vacuum inductance will rise to much higher values during the energy discharge pulse. The magnetic cores do not need to withstand the full output voltage, as the individual vacuum insulators can flash over and protect them from much of it. The energy stored in the inductance of the spark gap switch, capacitor, etc., outside the vacuum inductor is not then available to the load, but this can be a small part of the circuit inductance. This suggests that in some designs the circuit that charges a vacuum inductor need not add voltages inductively on cores but could instead be a Marx. The relatively low inductor charging voltage could be withstood in air, and the high inductor discharge voltage could be allowed to cause the controlled breakdown of spark gaps between stages, possibly polarity-sensitive $\mathrm{SF}_{6}$ spark gaps. The only advantage of the IVA mentioned in Sec. III A that would be largely lost is the suppression of external electric interference.

The last two IVAs to be mentioned here are both repetitive systems. CLIA (Compact Linear Induction Accelerator [71]) was built to drive repetitive high power microwave sources, principally relativistic magnetrons. The $750 \mathrm{kV}, 10 \mathrm{kA}, 100 \mathrm{~ns}$ output $(75 \Omega)$ was provided by a ten-stage Metglas-core induction adder. The microwave-producing load is necessarily in vacuum. However, at this output voltage and current, the advantage discussed in Sec. III A of reducing the total vacuum inductance by reducing the individual vacuum insulator voltages is not significant, and the bore of the IVA could be vacuum or could be oil with a full voltage $(750 \mathrm{kV})$ vacuum insulator. The vacuum approach was in fact chosen in CLIA. Magnetic insulation is undesirable when driving microwave source loads, as the electron sheath in the adder may not couple to the load. Hence the vacuum bore was designed to operate, with negative output, at fields $(\sim 200 \mathrm{kV} / \mathrm{cm})$ at which electron emission is small.

The individual stages of CLIA are driven by magnetic pulse compressors and can operate in bursts of up to hundreds of pulses at repetition rates of hundreds of pulses per second. In a magnetic pulse compressor [72], an inherently repetitive, long life switch such as a thyratron or solid-stage switch transfers energy from a dc capacitor in a time of a few to many microseconds pulse duration. Then a magnetic core like that in the IVA cells 
saturates and acts as a series switch to discharge the energy to another capacitor in a shorter time. A number of such magnetic-switched stages can be used to generate output pulses of $100 \mathrm{~ns}$ or less, the last switch usually discharging a water-dielectric coax (like that used in RITS and in other IVAs described earlier) in order to deliver a rectangular pulse. The lifetime of a magnetic pulse compressor can be that of the initiating thyratron or solid-state switch, $>10^{8}$ shots if desired, since the magnetic core switches themselves involve no discharges and only modest heating and have indefinitely long lives. Similarly the repetition rate can be very high, limited either by recovery of the initiating switch or by the time to recharge the energy store or to reset cores; it can easily exceed $1 \mathrm{kHz}$. The liquid dielectric switches described earlier in IVA drivers are by contrast essentially single pulse switches, although in the hundreds of $\mathrm{kV}$ range they can be operated at up to $\sim 1 \mathrm{kHz}$ in burst mode by using very powerful pumps to flow the liquid at very high speed [73]. Gas switches can also be used to drive IVAs, but for these also the repetition rates are limited, although again $1 \mathrm{kHz}$ has been obtained in burst mode in the case of relatively low energy LIA drivers using powerful flow systems. Also $>1 \mathrm{kHz}$ may be possible without flow even at larger energies, using hydrogen gas switches [74]. However, the life of liquid or gas spark gaps is relatively limited, and hence in high repetition rate or continuously operating drivers for IVAs the magnetic modulator is greatly superior. Provided the magnetic switches have cooling channels, continuous operation at $100 \mathrm{~Hz}$ or more has been demonstrated at up to $500 \mathrm{kV}$ in RHEPP [43] and in the PSI SLIA program (unpublished).

In CLIA, repetition rates of $250 \mathrm{~Hz}$ were obtained at switch voltages of $150 \mathrm{kV}$. Each of the ten $75 \mathrm{kV}$ CLIA cells was driven by a water pfl charged to $150 \mathrm{kV}$. The ten pfls were all charged by a common two-stage magnetic modulator incorporating a 2:1 transformer and ring-up from a large $40 \mathrm{kV}$ dc capacitor switched by a thyratron.

The cores in the switches and in the adder used 2605CO Metglas. At first the cores were wound with $3.5 \mu \mathrm{m}$ Mylar insulation, but discharges within the cores led to the substitution of $8 \mu \mathrm{m}$ paper. For the CLIA burst length of hundreds of pulses the thermal inertia of the cores was adequate and slow cooling of external cooling between bursts was all that was required.

The final IVA example is the RHEPP-II electron beam source $[43,75]$. This is a continuously operating $100 \mathrm{~Hz}$ system and therefore also uses magnetic modulator drive. Cooling channels are provided in the cores of all magnetic switches, transformers, and adder cells to remove heat in an equilibrium condition. The RHEPP program has used as the input stage to its magnetic compressor both an alternator (with $\sim 8 \mathrm{~ms}$ output) and SCR-switched modulators (silicon controlled rectifier) ( $>100 \mu \mathrm{s}$ ). Magnetic compression (five stages in the SCR case finally used) produces a $220 \mathrm{kV}, 50$ ns power pulse from a water dielectric triaxial pfl. This is delivered to a ten-stage IVA by fifty $22 \Omega$ polyethylene dielectric cables.

Efficiency was a major driver in the design of RHEPPII. The overall efficiency obtained was estimated as $76 \% \pm 20 \%$. As discussed in Sec. III C 4, losses in cores increase as the pulse duration is reduced, and to reduce loss the RHEPP IVA cores were made not of $2605 \mathrm{CO}$ Metglas but of 2605-TCA Metglas. Use of the flux swing of only $\sim 1 \mathrm{~T}$ available in 2605-TCA meant that the cores were larger, but the remanence is so small that no reset was needed.

The adder bore of RHEPP $(\sim 27 \mathrm{~cm}$ radius for 2.2 MV) operates at stresses similar to that of the vacuum bore of CLIA ( $9 \mathrm{~cm}$ radius for $750 \mathrm{kV}$ ). However, the bore insulation chosen in RHEPP-II is not vacuum but oil, with a full voltage vacuum insulator at the bore output-one of the schemes originally suggested in Ref. [24]. The reason stated for this choice in RHEPP was uncertainty about a long-term repetitive operation of MITLs; another disadvantage of magnetic insulation is the loss in efficiency if some of the electron sheath flow in the adder does not couple efficiently to the diode. For example, a $>1 \mathrm{~m}$ wide rectangular cathode has been used in RHEPP to generate a large area beam; electron sheath flow could not be directed into such a beam profile. As discussed in Sec. III B 2, sheath flow can be reduced by mismatching a magnetically insulated adder, but here this would trade one form of inefficiency for another. The purpose of RHEPP-II is to investigate various types of radiation processing, which could likely require using various beam and cathode shapes. This is possible to do efficiently using a full-voltage insulator stack with fields on the cathode conductor in vacuum that are below emission threshold. The other alternative would be to use such low fields throughout a vacuum bore in the IVA, which might require a somewhat larger bore than used on RHEPP-II. However, with the beam current of only $25 \mathrm{kA}$ in RHEPP-II the inductance penalty of a full voltage insulator (Sec. III A) following a liquid-insulated bore is small.

Both the IVA and LIA are candidates for applications in industrial radiation processing using their electron beams or the $\mathrm{x}$ rays these beams can produce. They can both operate continuously and reliably, as present technology has demonstrated at least for short periods. However, at present, such industrial applications require of the order of $100 \mathrm{~kW}$ of beam and this is most cheaply produced by continuous, not pulsed, accelerators. The applicability of induction systems depends on much higher average power being required or on processes being found for which a short pulse is beneficial.

To consider at what power levels induction systems could be used effectively in such industrial applications, consider first the IVA. Whatever bore insulation is used, the characteristic impedance of the bore of an IVA cannot 
be much greater than the $\sim 90 \Omega$ of RHEPP-II. That means that if IVAs are used in the 5-10 MV range that is desired for commercial radiation processing, the diode power will be $0.25-1 \mathrm{TW}$ and the energy in a $50 \mathrm{~ns}$ pulse will be 10 to $50 \mathrm{~kJ}$. (Mismatching the adder into a higher impedance diode is possible, but is not efficient for a long 5 or $10 \mathrm{MV}$ adder.) Operating such IVAs at the rates of hundreds of $\mathrm{Hz}$ that they are capable of would deliver many $\mathrm{MW}$ to tens of MW of beam. If powers in the range of hundreds of $\mathrm{kW}$ to a few megawatts are needed, the desire to minimize the size and cost of the accelerator and hence the capital investment by operating at the largest possible repetition rate, perhaps around $1 \mathrm{kHz}$, will probably favor the use of the LIA, where the energy per pulse can be lower. IVAs will be advantageous if the power range approaches or exceeds $10 \mathrm{MW}$.

[1] N. Christofilos et al., Rev. Sci. Instrum. 35, 886 (1964).

[2] J. N. Downing et al., in Proceedings of the 8th IEEE Pulsed Power Conference, San Diego, CA, 1991 (IEEE, Piscataway, NJ, 1991), p. 949.

[3] D. Weidenheimer et al., in Proceedings of the 13th IEEE Pulsed Power Conference, Las Vegas, NV, 2001 (IEEE, Piscataway, NJ, 2001), Vol. 1, p. 591.

[4] James E. Leiss, Part. Accel. 10, 223 (1980).

[5] R. B. Miller et al., J. Appl. Phys. 51, 3506 (1980).

[6] E. E. Jones et al., in Proceedings of the 6th IEEE Pulsed Power Conference, Arlington, VA, 1987 (IEEE, Piscataway, NJ, 1987), p. 498.

[7] M. G. Mazarakis et al., in Proceedings of the 8th IEEE Pulsed Power Conference, San Diego, CA, 1991 (Ref. [2]), pp. 26-29.

[8] A. I. Pavlovskii et al., Sov. Phys. Dokl. 20, 441 (1975).

[9] A. I. Pavlovskii et al., in Proceedings of the 9th International Conference On High Power Particle Beams, Washington, DC, 1992 (IEEE, Piscataway, NJ, 1992), p. 441.

[10] D. Eccleshall and J. K. Temperly, J. Appl. Phys. 49, 3649 (1978).

[11] D. L. Smith et al., in Proceedings of the 8th IEEE Pulsed Power Conference, San Diego, CA, 1991 (Ref. [2]), p. 945.

[12] I. Smith, in Proceedings of the IEEE Conference Record of 1982 Fifteenth Power Modulator Symposium, Baltimore, MD (IEEE, Piscataway, NJ, 1982), pp. 223226.

[13] V. S. Bossamykin et al., in Proceedings of the 9th IEEE Pulsed Power Conference, Albuquerque, NM, 1993 (IEEE, Piscataway, NJ, 1993), p. 905.

[14] V.S. Bossamykin et al., in Proceedings of the 9th IEEE Pulsed Power Conference, Albuquerque, NM, 1993 (Ref. [13]), p. 910.

[15] Ian Smith, Rev. Sci. Instrum. 50, 714 (1979).

[16] V.S. Bossamykin et al., in Proceedings of the 9th IEEE Pulsed Power Conference, Albuquerque, NM, 1993 (Ref. [13]), p. 901.
[17] D. Birx, E. Cook, S. Hawkins, S. Poor, L. Reginato, J. Schmidt, and M. Smith, IEEE Trans. Nucl. Sci. 30, 2763 (1983).

[18] I. Smith and D. Birx, ONR Report No. PSI-FR-21-167, 1984.

[19] H. Kirbie et al., in Proceedings of the 10th IEEE Pulsed Power Conference, Albuquerque, NM, 1995 (IEEE, Piscataway, NJ, 1995), p. 447.

[20] Leland G. Schlitt et al., ONR Report No. PSI-FR-21-167, 1984.

[21] S. D. Putnam, in Proceedings of the IEEE Particle Accelerator Conference, Washington, DC, 1987 (IEEE, Piscataway, NJ, 1987), p. 887.

[22] J. R. Smith et al., in Proceedings of the 1997 Particle Accelerator Conference, Vancouver, BC, Canada (IEEE, Piscataway, NJ, 1997), p. 1251.

[23] Donald Eccleshall et al., in Proceedings of the Particle Accelerator Conference, San Francisco, CA, 1979 (IEEE, Piscataway, NJ, 1979), p. 4245; also J. Appl. Phys. 49, 3649 (1978).

[24] I. D. Smith, "PIB Reactor-Driver Design Based on Small Sub-Modules," Sandia Technical Note, 1979 (unpublished).

[25] B. Bernstein and I. Smith, IEEE Trans. Nucl. Sci. 20, 294 (1973).

[26] J. J. Ramirez et al., in Proceedings of the 7th IEEE International Pulsed Power Conference, Arlington, VA, 1985 (IEEE, Piscataway, NJ, 1985), p. 143.

[27] B. Kulke, D. S. Ravenscroft, and G. E. Vogtlin, IEEE Trans. Nucl. Sci. 28, 2882 (1981).

[28] Ian D. Smith et al., IEEE Trans. Plasma Sci. 28, 1653 (2000).

[29] A.V. Gordeev, Report No. IAE-3076, Moscow, 1978, pp. 1-63.

[30] I. Smith et al., in Proceedings of the 12th IEEE International Pulsed Power Conference, Monterey, CA, 1989 (IEEE, Piscataway, NJ, 1989), p. 36.

[31] J. M. Creedon, Appl. Phys. 48, 1070 (1977).

[32] T. P. Hughes et al., Phys. Rev. ST Accel. Beams 2, 110401 (1999).

[33] D. Rovang et al., in Proceedings of the 14th IEEE Pulsed Power Conference, Dallas, Texas, 2003 (IEEE, Piscataway, NJ, 2003).

[34] A. R. Birrell et al., IEEE Trans. Plasma Sci. 28, 1660 (2000).

[35] V. Bailey, I. Smith, T. Goldsack, K. Thomas, and J. Maenchen, in Proceedings of the 2002 IEEE International Conference of Plasma Sciences, Banff, Alberta, Canada (IEEE, Piscataway, NJ, 2002), p. 326.

[36] C.W. Mendel et al., Laser Part. Beams 1, 311 (1983).

[37] J. Ramirez, K. Prestwich, and I. Smith, Proc. IEEE 80, 946 (1992).

[38] J. P. Corley et al., in Proceedings of the 7th IEEE Pulsed Power Conference, 1989 (Ref. [30]), pp. 571-574.

[39] D. L. Johnson et al., in Proceedings of the 7th IEEE Pulsed Power Conference, 1989 (Ref. [30]), pp. 32-35.

[40] J. P. Corley et al., in Proceedings of the 8th IEEE Pulsed Power Conference, San Diego, CA, 1991 (Ref. [2]), pp. 920-923.

[41] J. P. Vandevender (private communication). 
[42] G. B. Frazier, in Proceedings of the 2nd IEEE Pulsed Power Conference, Lubbock, TX, 1979 (IEEE, Piscataway, NJ, 1979), p. 127.

[43] L. Schneider et al., in Proceedings of the 12th IEEE Pulsed Power Conference, Monterey, CA, 1999 (IEEE, Piscataway, NJ, 1999), p. 523.

[44] J. J. Ramirez, in Proceedings of the 10th IEEE Pulsed Power Conference, Albuquerque, NM, 1995 (Ref. [19]), p. 91.

[45] C. H. Smith and L. Barberi, in Proceedings of the 7th IEEE International Pulsed Power Conference, Arlington, VA, 1985 (Ref. [26]), p. 644.

[46] P. Corcoran et al., in Proceedings of the IEEE Particle Accelerator Conference, San Francisco, CA, 1991 (IEEE, Piscataway, NJ, 1991), p. 3114; IEEE Catalog No. 91CH3038-7, p. 3114.

[47] P. Anthouard et al., in Proceedings of the 23rd International Power Modulator Symposium, Rancho Mirage, CA, 1998 (IEEE, Piscataway, NJ, 1998), p. 80.

[48] J. C. Martin, AWRE Report No. SSWA, JCM/713/157, 1971; in Pulsed Power, edited by T. H. Martin, A. H. Guenther, and M. Kristiansen (Plenum Press, New York, 1996), p. 255.

[49] W. A. Stygar et al., in Proceedings of the 12th IEEE Pulsed Power Conference, Monterey, CA, 1999 (Ref. [43]), p. 454.

[50] Ian Smith, IEEE Trans. Plasma Sci. 25, 293 (1997); see also Proceedings of the 10th IEEE Pulsed Power Conference, Albuquerque, NM, 1995 (Ref. [19]), p. 558.

[51] D. L. Johnson et al., in Proceedings of the 13th IEEE Pulsed Power Conference, Las Vegas, NV, 2001 (Ref. [3]), p. 600.

[52] J. J. Ramirez et al., in Proceedings of Beams 88, Karlsruhe (IEEE, Piscataway, NJ, 1988), p. 148.

[53] Carl Ekdahl, in Proceedings of the 13th IEEE Pulsed Power Conference, Las Vegas, NV, 2001 (IEEE, Piscataway, NJ, 2001), p. 29.

[54] R. Scarpetti (private communication).

[55] K. Thomas (private communication).

[56] J. P. Corley et al., in Proceedings of the 6th IEEE Pulsed Power Conference, Arlington, VA, 1987 (Ref. [6]), p. 486.

[57] I. Smith et al., in Proceedings of the 14th IEEE Pulsed Power Conference, Dallas Texas, 2003 (Ref. [32]), Vol. 1, p. 371.

[58] B. N. Turman et al., in Proceedings of the 7th IEEE Pulsed Power Conference, 1989 (Ref. [30]), p. 553.
[59] P. R. Menge et al., in Proceedings of the 13th IEEE Pulsed Power Conference, Las Vegas, NV, 2001 (Ref. [3]), p. 454.

[60] K. J. Thomas et al., in Proceedings of the 13th IEEE Pulsed Power Conference, Las Vegas, NV, 2001 (Ref. [3]), p. 306.

[61] M. Mazarakis et al., in Proceedings of the 11th IEEE Pulse Power Conference, Baltimore, MD, 1997 (IEEE, Piscataway, NJ, 1997), p. 642.

[62] E. Buszen et al., in Proceedings of the 6th IEEE Pulsed Power Conference, Arlington, VA, 1987 (Ref. [6]), p. 506.

[63] John R. Smith et al., in Proceedings of the 13th IEEE Pulsed Power Conference, Las Vegas, NV, 2001 (IEEE (Ref. [53].

[64] K. J. Thomas et al., in Proceedings of the 12th IEEE Pulsed Power Conference, Monterey, CA, 1999 (Ref. [43]), p. 211.

[65] J. P. Corley et al., in Proceedings of the 10th IEEE Pulsed Power Conference, Albuquerque, NM, 1995 (Ref. [19]), p. 1461.

[66] K.W. Struve et al., in Proceedings of the 12th IEEE Pulsed Power Conference, Monterey, CA, 1999 (Ref. [43]), p. 493.

[67] I. D. Smith et al., in Proceedings of the 11th IEEE Pulsed Power Conference, Baltimore, MD, 1997 (Ref. [61]), p. 168.

[68] A. A. Kim et al., in Proceedings of the 13th IEEE Pulsed Power Conference, Las Vegas, NV, 2001 (Ref. [3]), p. 1491.

[69] I. Smith et al., in Proceedings of the 13th IEEE Pulsed Power Conference, Las Vegas, NV, 2001 (Ref. [3]), p. 582.

[70] A. N. Bastrikov et al., in Proceedings of the 11th IEEE Pulse Power Conference, Baltimore, MD, 1997 (Ref. [61]), p. 489.

[71] S. Ashby et al., in Proceedings of the 9th IEEE Pulse Power Conference, Albuquerque, NM, 1993 (Ref. [13]), p. 940.

[72] W. S. Melville, Proc. IEEE 98, 185 (1951).

[73] P. Champney et al., in Proceedings of the 8th IEEE Pulsed Power Conference, San Diego, CA, 1991 (Ref. [2]), p. 863.

[74] M. G. Grothaus et al., in Proceedings of the 9th IEEE Pulsed Power Conference, Albuquerque, NM, 1993 (Ref. [13], p. 475.

[75] D. L. Johnson et al., in Proceedings of the 10th IEEE Pulse Power Conference, Albuquerque, NM, 1995 (Ref. [19]), p. 437. 